\title{
Simultaneous measurements of room-acoustic parameters using different measuring equipment?
}

\author{
Halmrast, Tor; Gade, Anders Christian; Winsvold, Bjorn
}

Published in:

Acoustical Society of America. Journal

Link to article, DOI:

$10.1121 / 1.422575$

Publication date:

1998

Document Version

Publisher's PDF, also known as Version of record

Link back to DTU Orbit

Citation (APA):

Halmrast, T., Gade, A. C., \& Winsvold, B. (1998). Simultaneous measurements of room-acoustic parameters using different measuring equipment? Acoustical Society of America. Journal, 103(5), 3034-3034.

https://doi.org/10.1121/1.422575

\section{General rights}

Copyright and moral rights for the publications made accessible in the public portal are retained by the authors and/or other copyright owners and it is a condition of accessing publications that users recognise and abide by the legal requirements associated with these rights.

- Users may download and print one copy of any publication from the public portal for the purpose of private study or research.

- You may not further distribute the material or use it for any profit-making activity or commercial gain

- You may freely distribute the URL identifying the publication in the public portal

If you believe that this document breaches copyright please contact us providing details, and we will remove access to the work immediately and investigate your claim. 


\title{
Session 5aPLa
}

\section{Plenary Lecture}

\author{
Andrea Prosperetti, Chair \\ Mechanical Engineering, Johns Hopkins University, 122 Latrobe Hall, 3400 North Charles Street, Baltimore, \\ Maryland 21218 \\ Chair's Introduction-8:00 \\ Invited Paper \\ 8:05
}

5aPLa1. Acoustics of two-phase fluids and sonoluminescence. Robert I. Nigmatulin (Ufa (Bashkortostan) Branch of Russian Acad. of Sci., K. Marx Str. 6, Ufa 450000, Russia, iskander@ncan.ufanet.ru)

The basic equations for dynamics of two-phase mixtures like gas-particle suspensions and bubbly liquids are presented. Heat and mass exchange phenomena near a drop, particle, and gas or vapor bubble are discussed. It is shown that these phenomena play an important role in propagation, attenuation, and amplification of sound waves and shock waves in gas-liquid systems, sometimes leading to paradoxical effects. The dynamics of a sonoluminescing bubble is discussed. There are two stages of the bubble oscillation process: The low Mach number stage when the velocity of the bubble interface is small compared with sound speed in the liquid, and the stage corresponding to the collapsing bubble's compression, when the velocity of the interface may be larger than the sound speed. The analytic solution for the low Mach stage is presented. This solution provides the economical boundary condition around the bubble for an effective numerical code.

FRIDAY MORNING, 26 JUNE 1998

CASCADE BALLROOM II (W), 7:20 TO 10:45 A.M.

\section{Session 5aAA}

\section{Architectural Acoustics: Case Studies of Performance Spaces}

\author{
Jerald R. Hyde, Chair \\ Consultant in Acoustics, Box 55, St. Helena, California 94574
}

Chair's Introduction-7:20

Invited Papers

5aAA1. The Hong Kong Cultural Centre halls: Acoustical design and measurements. A. Harold Marshall, Johan L. Nielsen, and M. Miklin Halstead (Acoust. Res. Ctr., Univ. of Auckland, New Zealand)

The main performance spaces in the Hong Kong Cultural Centre comprise the Concert Hall, seating 2000 and the Grand Theatre seating 1700. The design of the former is derived from the Christchurch Town Hall which it develops in several important ways, while the latter is a lyric theatre/opera hall with several unique features which are described briefly in this paper. The Centre opened in 1989 following extensive commissioning measurements in the halls. Recently the opportunity has arisen to repeat the measurements using an improved technique in an attempt to document changes following a stage extension. Results from these measurements are compared with the earlier series in each hall. Marshall Day Associates, Auckland, New Zealand was responsible for the room acoustical design of these spaces. [The recent visit to Hong Kong was supported by the Acoustics Research Centre, the Hong Kong Cultural Centre, and the Marsden Fund administered by the Royal Society of New Zealand.]

\section{$7: 45$}

5aAA2. Modern measurements, optimized diffusion, and electronic enhancement in a large fan-shaped auditorium. John $P$. O'Keefe (Aercoustics Eng. Ltd., 50 Ronson Dr., Ste. 127, Toronto, ON M9W 1B3, Canada), Trevor Cox (Univ. of Salford, UK), Neil Muncy (Neil Muncy Assoc., Toronto, Canada), and Steve Barbar (LARES Assoc., Cambridge, MA)

The Hummingbird Centre in Toronto Canada, formerly known as the O'Keefe Centre, is a 3000 seat fan-shaped auditorium, typical of the postwar era. Since it opened in 1960 it has been plagued by complaints about its acoustics. A LARES electronic enhancement system was proposed for the building and a feasibility study was carried out. Modern acoustical measurements lend 
credence to the years of complaints. Among other interesting findings, late energy (Glate) is $10-20 \mathrm{~dB}$ lower than a traditional shoe-box-shaped concert hall. Such low levels of late energy explain, in part, the image shift and echoes that are found throughout the hall. A side wall echo threatened to compromise the proposed enhancement system. In an effort to reduce the echo, a crescent-shaped diffuser was developed using a BEM optimization routine. Four LARES mainframes are employed in the enhancement system with more than 280 front ported two-way loudspeakers, each of which can be addressed individually. The renovated acoustics have been well received by the owners, users, and patrons.

\section{Contributed Papers}

\section{8:05}

5aAA3. Recent acoustical measurements in the Christchurch Town Hall Auditorium. A. Harold Marshall, Johan L. Nielsen, and M. Miklin Halstead (Acoust. Res. Ctr., Univ. of Auckland, Private Bag 92019, Auckland, New Zealand, h.marshall@auckland.ac.nz)

Two significant changes have occurred in the Christchurch Town Hall during 1996-97: the original seats have been replaced with a new type and a new Rieger pipe organ has been installed behind the choir seats. A new comprehensive set of acoustic measurements has been made in the unoccupied hall. These are reported and compared in this paper with earlier measurements. A related study on the usefulness of C80 as a clarity measure in concert halls and which makes use of these results is reported in a companion paper. [This work is supported by the Marsden Fund administered by the Royal Society of New Zealand.]

\section{8:20}

5aAA4. Calculation and measurement of acoustic factors for the Kirishima International Concert Hall. Tatsumi Nakajima (Takenaka Res. and Development Inst., 5-1, 1-chome Ohtsuka, Inzai, Chiba, 270-13 Japan) and Yoichi Ando (Kobe Univ., Rokkodai, Nada, Kobe, 657 Japan)

The Kirishima International Concert Hall was designed by architect F. Maki with Ando's acoustic design theory. It opened on July 22, 1994. The 770 seat main hall which has a volume of $8475 \mathrm{~m}^{3}$ was primarily designed for chamber music. Computer simulations made it clear that the acoustical design of the main hall would be an optimum solution with a plan like a leaf shape and a cross section like a bottom of a ship. These were solutions which satisfied requirements for both acoustical and architectural design concepts of the main hall. Getting a low IACC sound field at the front seat was most important. In order to get a low IACC, sound reflections from the stage to the audience floor were examined and studied in the early design processes. Calculation and measurement of acoustic factors of the sound fields in the main hall show: (1) the calculated and the measured IACCs are good in agreement, (2) IACCs tended lower values of about 0.1 than the calculated ones throughout the seats, and (3) the number of reflections needed for predicting IACC is recommended to be about 40 for the practical method of IACC calculation at the design stage of a hall.

\section{$8: 35$}

5aAA5. Interrelationship of musical excellence and acoustical excellence: A case study of the Gewandhaus, Leipzig. Pamela Clements (Acoust. Consultant, Jaffe Holden Scarbrough Acoust., Inc., 114A Washington St., Norwalk, CT 06854, pclements@jhsacoustics.com)

A study of changes in orchestral performance practice and repertoire in the Altes Gewandhaus and the Neues Gewandhaus (opened 1884) under the conductor Carl Reinecke was performed. A parallel study of changes in orchestral performance practice and repertoire in the Neues Gewandhaus was also performed when Carl Reinecke was succeeded by Arthur Nikisch in 1895. In the first instance, the orchestra and conductor are the same but the hall changes; in the second instance, the orchestra and hall are the same but the conductor changes. Both old and new halls were regarded as outstanding acoustically, but under Reinecke the orchestra's reputation waned because of conservatism in programming and interpretation. Historical evidence shows that the orchestra went through a difficult period accommodating the new hall's acoustics and emerged with a new sound. Yet, despite the hall's excellent acoustics, the orchestra performed better in another, poorer, acoustic environment under the more dynamic conductor Arthur Nikisch. The orchestra only regained its international reputation later when Nikisch became conductor at the
Gewandhaus. The study explores Nikish's response to the hall's acoustic possibilities, and suggests reasons why a hall with a relatively dry acoustic could be pivotal in the orchestra's then becoming a foremost interpreter of the late Romantic repertoire.

\section{8:50}

5aAA6. Acoustical design and the characteristics of Sapporo Concert Hall. Yasuhisa Toyota and Katsuji Naniwa (Nagata Acoust., Inc., Minami-Shinjuku-Hoshino Bldg. 8F, 5-23-13 Sendagaya, Shibuya-ku, Tokyo, 151-0051 Japan, toyota@ nagata.co.jp)

Sapporo Concert Hall, with 2008 seats in the large hall and 453 seats in the small hall, was opened on July 4, 1997. Both halls were designed for classical music, the large one mainly for orchestral music and the small one for chamber music and recitals. The large hall was designed with the concept of the surrounded stage and "vineyard steps" seating arrangement like Philharmonic Hall in Berlin and Suntory Hall in Tokyo. The emphasized points in acoustical design were as follows: (1) Effective use of the "vineyard steps" walls which were introduced into the audience area to provide the effective early reflections; (2) installation of the big sound reflectors suspended above the stage area to provide the effective early reflections to musicians and audience around the stage; (3) introduction of heavy material, 150-millimeters-thick concrete, into the main ceiling to get enough response in the low frequencies. The small hall was designed as a typical shoebox hall with narrow width and high ceiling. Acoustically tunable curtains were introduced to make the wide range of acoustical possibilities. The results of the acoustical design and its characteristics will be reported.

\section{9:05}

5aAA7. Acoustical renovation of The Opheum Theatre, Vancouver Canada. John P. O'Keefe (Aercoustics Eng. Ltd., 50 Ronson Dr., Ste. 127, Toronto, ON M9W 1B3, Canada), Gilbert A. Soulodre (Commun. Res. Ctr., Ottawa, ON K2H 8S2, Canada), and John S. Bradley (Natl. Res. Council, Ottawa, ON K1A 0R6, Canada)

The Orpheum Theatre is a 2800 seat vaudeville house that was renovated for the Vancouver Symphony in the 1970s. Funds ran out prior to completion and some problematic conditions remained for the following fifteen years. Perhaps the most significant was an image shift heard on the balcony. Two focused reflections arriving at approximately $50 \mathrm{~ms}$ made some source locations on the stage appear to be perched in the ceiling. The efficacy of the plastic reflectors above the stage was also questioned. These and other issues were addressed with a complete set of stage and audience measurements in the full-scale room, experiments with a 1:48 small scale model, and listening tests using anechoic music convolved with the full-scale binaural impulse responses. The latter was particularly important because it allowed for reliable quantification of image shift thresholds that were unique to this room. Small scale modeling proved useful in matching the new convex reflectors' radii of curvature to the image shift threshold. The renovation work is complete and the image shift has been completely eradicated.

\section{9:20-9:30 Break \\ 9:30}

5aAA8. The best remaining seat. Bodil Vaupel (Arkitekturt Akuslik, Paludan Müllers Plads 1, 5300 Kerteminde, Denmark)

It is important that an auditorium be designed to have as many good seats as possible. Not all seats in an auditorium are equally good. This is manifested in that the audience does not choose its seats randomly. The audience understands intuitively that, generally speaking, the closer a seat 
is, and the more straight on, the better it is. Imagine an auditorium with open seating and the audience entering one at a time. People will in turn make a selection of what in their opinion is the best remaining seat. The order in which the seats are chosen is an indicator of the rank order of the desirability of the individual seats. As the audience makes its seat selection, a geometric pattern of the occupied seats unfolds, and reveals the boundary of the preferred seats. The perimeters describe the equal desirability curves and outlines the auditorium plan with as many good seats as possible. The audience choice of seat is recorded by time lapse photography. The data are analyzed in a combined computer drawing and mathematics program. A mathematical model has been developed that evaluates the desirability of the seating in an auditorium from the audience's point of view.

\section{9:45}

5aAA9. Acoustic design and performance of the Bruce Mason Theatre. Joanne M. Valentine (Marshall Day Asociates, P.O. Box 5811, Wellesley St., Auckland 1, New Zealand, mda@bitz.co.nz)

The Bruce Mason Theatre takes an innovative approach to variable acoustics with the use of a variable volume operable ceiling. The overall acoustic design objective was to achieve excellent acoustics with a high level of reverberance for symphony, and good speech clarity with a reduced level of reverberance for theater and drama. The results of commissioning measurements carried out in the auditorium show that a high degree of variability between the acoustic conditions for symphony mode and theater mode has been achieved, and that the operable ceiling/variable volume concept has proved to be highly successful. This paper presents the design concept developed for the auditorium, the findings of a 1:25 scale acoustic model study, and the results of commissioning measurements carried out in the newly opened theater in September 1996.

\section{0:00}

5aAA10. Three-dimensional impulse response measurements on $\mathrm{S}$. Maria Church, Florence, Italy. Angelo Farina (Dipartimento di Ingegneria Industriale, Viale delle Scienze, 43100 Parma, Italy, farina@pcfarina.eng.unipr.it) and Lamberto Tronchin (DIENCACIARM Univ. of Bologna, Viale Risorgimento, 240136 Bologna, Italy)

The measurement of binaural impulse response procedure for acoustic environments has been established and achieved from the International Standard Organization; following the ISO 3382 many acoustical parameters have been already analyzed in the past, ranging from Royal Albert Hall in London, UK, to Teatro la Fenice in Venice, Italy. In this paper a new procedure for measuring 3D impulse response has been utilized, with the aim to reconstruct the $3 \mathrm{D}$ sound field especially for virtual reality purposes. Starting from the definition of B-format impulse responses, the pressure gradient has been measured in the Church by using a homemade apparatus and software, and performing measurements on the three axes, gathering seven different impulse responses, without using expensive microphones already developed. From the measures, the relations between the seven different measurements have been found out, and the results have been compared with the binaural impulse responses measurements performed in the same position at the same time.

\section{0:15}

5aAA11. Simultaneous measurements of room-acoustic parameters using different measuring equipment? Tor Halmrast (Statsbygg, pb. 8106 Dep. N-0032, Oslo, Norway), Anders Gade (Tech. Univ. of Denmark, DK-2600 Lyngby, Denmark), and Bjorn Winsvold (Norsonic, pb. 24, N-3408, Tranby, Norway)

Often the results from different room-acoustic measurements in the same hall disagree, and the disagreement is just said to be due to different measuring equipment, or different rigging/temperature, etc. The room acoustic of the Oslo Concert Hall was measured simultaneously, using the following different measuring equipment: (1) MLS/MLSSA (Statsbygg), (2) Sweep-Tone (Tech. Univ. Denmark), and (3) Norsonic 840 with MLS + MatLab. For some of the measurements (4) Pistol and (5) Electrical Impulse were also used. The paper will compare the results from the different measuring equipment, for the most known room-acoustic parameters. For the reverberation time parameters RT and EDT, very good agreement was found between the three main measuring equipments. For Ts and C80 the agreement between these three is good/fair for the higher frequencies, but less good for the bass, especially C80. The measurements with Electric Pulse and Pistol as signals (analyzed through Norsonic + MatLab) indicate good agreement for the reverberation times, but EDT is somewhat higher for the Pistol. For Ts and C80 the Electric Impulse and especially the Pistol give less clearness (higher Ts and lower C80), compared to MLSSA, Sweep Tone, and Norsonic/MLS.

\section{$10: 30$}

5aAA12. Design of a circular hall improving the subjective preference at each seat. Akio Takatsu, Hiroyuki Sakai, Shin-ichi Sato, and Yoichi Ando (Grad. School of Sci. and Technol., Kobe Univ., Rokkodai, Nada, Kobe, 657 Japan)

The circular type of the medium-sized (400 seats) multipurpose event hall in Kobe Fashion Plaza, which was named Orvis Hall, was designed based on Ando's theory of subjective preference. Acoustic problems caused by such a circular plan were minimized by means of ceiling diffusion panels, side-wall-reflective panels, and a small room at the back wall preventing echo-disturbance created by the long-path echo or "Whispering Gallery" effects. One of the most remarkable systems of this hall is controlling the subsequent reverberation time through blending architectural acoustics and electrical acoustics by use of a hybrid system with both a reverberation control room and a digital reverberator. Therefore the total scale value of subjective preference at each seat is maximized by the four acoustic factors, namely, the delay time of first reflection, the listening level, the subsequent reverberation time, and the IACC. After construction of this hall, four factors at each seat were measured, and results are presented. 


\title{
Session 5aAO
}

\section{Acoustical Oceanography and Animal Bioacoustics: Acoustics of Fisheries and Plankton IV}

\author{
John Hedgepeth, Chair \\ BioSonics, Inc., 4027 Leary Way, NW, Seattle, Washington 98107
}

\section{Invited Paper}

\begin{abstract}
10:00
5aAO1. Vessel avoidance of wintering Norwegian spring spawning herring. Rune Vabø (Inst. of Marine Res., P. O. Box 1870, N-5024 Bergen, Norway), Kjell Olsen (Univ. of Troms $\varnothing$, School of Fisheries, N-9000 Troms $\varnothing$, Norway), and Ingvar Huse (Inst. of Marine Res., Bergen, Norway)

Vessel avoidance during acoustic abundance estimation of herring was investigated. Echo energy values measured from a submerged 38-kHz transducer were recorded and values at the time of passage were compared with corresponding echo energy values from a hull-mounted $18-\mathrm{kHz}$ transducer on the passing vessel. A quantification of the loss in echo energy due to avoidance behavior is presented. Experiments are grouped into nighttime and daytime experiments. While a considerable drop in echo energy at the time of passage was observed at night, insignificant loss was found during the day. A general avoidance reaction pattern was identified in all the nighttime experiments. The magnitude of the vessel avoidance reaction was found to be strongly dependent on depth. Between 40 and 100-m depth some nighttime experiments showed loss in echo energy of the order of $90 \%$. The effect of avoidance behavior decreased with depth below $100 \mathrm{~m}$, but was seen down to $150 \mathrm{~m}$ at night. Low daytime response is due to deep daytime herring distribution. Body tilting and vertical and horizontal swimming are suggested explanations for the lower echo energy during passage.
\end{abstract}

\section{Contributed Papers}

\section{0:20}

5aAO2. FishMASS: ADCP technology adapted to split-beam fisheries echo sounding. R. Lee Gordon (RD Instruments, 9855 Businesspark Ave., San Diego, CA 92131, lgordon@ rdinstruments.com) and Len Zedel (Memorial Univ. of New Foundland, Canada)

The objective of FishMASS, a NSF-funded research project, is to adapt elements of ADCP technology into split-beam echo sounding. Improvements come from wide bandwidths (50\% bandwidth at $300 \mathrm{kHz}$ ), incorporation of velocity (Doppler) data, and addition of autonomous operation (battery and recording capacity to allow operation for up to a year). Wide bandwidths can reduce the uncertainty of single-target echoes from 3-6 dB (characteristic of narrowband signals) down to a fraction of a dB. It enables better differentiation of single-target from multiple-target echoes (when targets are separated either radially or at different ranges). The $50 \%$ bandwidth gives access to at least part of the target echo spectrum. These three advantages combine to improve both target discrimination and target classification. Split-beam sonars can sometimes track individual targets through their beams to obtain swimming speed. Doppler processing (identical to the processing of broadband ADCPs) will be added to obtain single-ping swimming speeds, both of individuals and groups. Mean velocities allow observation of migration and flux. Statistical products such as velocity standard deviation enable observation of feeding or predatoravoidance behavior. Correlations of velocity with, for example, TS may further aid species classification.

\section{0:35}

5aA03. The digital transducer, new sonar technology. William Acker (BioSonics, Inc., 4027 Leary Way NW, Seattle, WA 98107)

The new digital architecture in sonar technology designed by BioSonics, Inc. has many advantages when compared with traditional analog technology. The echo signal is digitized inside the transducer at a very low signal level. This removes problems of coupled or induced noise when sending the signal through long lines. The system operates with a 3 to 4 $\mathrm{dB}$ noise figure in all modes of operation. Another important feature of the system is very high dynamics ( $>132 \mathrm{~dB}$ continuous). This allows receiving of low signals from small targets (i.e., plankton) simultaneously with high level signals (i.e., bottom) without saturation. The echo signal is sampled at a high rate and stored with high accuracy $(0.013 \%)$ and high resolution (1.8 cm or less). The sonar is PC controlled for simple operation. The large dynamic range means no adjustment in system gain or transmit power. The data acquisition system is virtually automatic because most decisions are in postprocessing. Raw data is stored on the PC's hard drive or similar device. The sonar is available in a wide frequency range $(38 \mathrm{kHz}-1 \mathrm{MHz})$ and single, dual, or split beam transducer configuration depending on the application, i.e., biomass, layering effects, bottom typing, etc.

\section{0:50}

5aA04. An acoustic tag "radar-type" tracking system for fish behavior studies near structures. John Hedgepeth, Dave Fuhriman (BioSonics, Inc., 4027 Leary Way NW, Seattle, WA 98107, jhedgepeth@biosonicsinc.com), Robert Johnson, and David Geist (PNNL, Battelle Memorial Inst., Richland, WA)

Recent studies of fish behavior around hydroelectric dams have used acoustics with split-beam methodology. A complementary methodology called the tracking transducer takes advantage of split-beam capabilities for expanding fish behavior investigations. The principle of tracking radar, aligning the antenna beam with a target, was applied with an acoustic transducer and dual-axis rotators for tracking individual fish over long periods of time. Deviation of the target from the beam axis produces a correction to point the axis toward the target. Two of these tracking systems have been used to triangulate the position of a small acoustic transmitter implanted in salmonid fish in the forebay of Lower Granite on the Snake River. This paper describes the system design, development, and early implementation. In October 1997 a 6-mm-diameter 200-kHz acoustic tag was placed in a rainbow trout and fish movement was measured near a surface bypass collector. The tracking equipment consisted of two 201-kHz, 6 deg half-power, full-beamwidth transducers placed $12.2 \mathrm{~m}$ 
apart and $3.0 \mathrm{~m}$ below the water surface. Each split-beam transducer was mounted on a high-speed, dual-axis, motorized armature. These armatures were computer controlled and the system automatically followed the tag by using angle estimates provided by the echosounders.

\section{1:05}

5aAO5. Field trials using an acoustic buoy to measure fish response to vessel and trawl noise. Chris D. Wilson (Natl. Marine Fisheries Service, NOAA, Seattle, WA, cdwilson@afsc.noaa.gov)

A freely drifting acoustic buoy was constructed to evaluate the response of fish to vessel and trawl noise. The buoy contains an echosounder and split beam transducer operating at $38 \mathrm{kHz}$. Transducer heading data from the buoy are collected to assess the directivity of the fish response (i.e., movement relative to vessel and trawl). Geographic position of the buoy is monitored via GPS. Data are stored onboard the buoy and telemetered directly to the support vessel. The radio link between the buoy and vessel is used to control the echosounder, receive buoy positions, and remotely generate echograms in real time. GPS data from the buoy are also transmitted via the Argos satellite system to the vessel to locate the buoy in the event that visual, radar, and direct radio contact are lost. Field trials include repeated passes by the NOAA research vessel Miller Freeman past the buoy at different ship speeds while free-running and trawling to determine the behavioral response of walleye pollock (Theragra chalcogramma) to vessel and trawl noise. Performance of the buoy and interpretation of the results will be discussed.
11:20

5aAO6. Noise characteristics of Japanese fisheries' research vessels. Yoshimi Takao, Kouichi Sawada, Yoichi Miyanohana, Tsuyoshi Okumura (Natl. Res. Inst. of Fisheries Eng., Ebidai Hasaki, Kashima Ibaraki, 314-04 Japan, ytakao@nrife.affrc.go.jp), Masahiko Furusawa (Tokyo Univ. of Fisheries, Tokyo, 108 Japan), and Doojin Hwang (Yosu Natl. Fisheries Univ., Yosu, Chonnam, 550-749, Korea)

Noise is one of the important problems for hydroacoustic surveys of fisheries resources, because it may cause a large error in estimated results and it may shorten detectable range. It is necessary to estimate and reduce the contribution of acoustic noise for precise and accurate surveys. Noise is classified into low-frequency noise (audible by fish) and high-frequency noise (affecting echo sounders). High-frequency noise usually results in an overestimate of acoustic return. The average power of the high-frequency noise was measured by the echo-integrator. The echo-integrator output was converted into the equivalent noise spectrum level which can be compared with noises received by other quantitative echo-sounding systems or environmental noises. The noise measurements of several fisheries research vessels, ranging from 29.5-m length (RV "Taka Maru") to 93-m length (RV "Kaiyo Maru"), were conducted to know their characteristics of noise. The dependence of noise upon the ship speed and the screw propeller's setting was investigated for each vessel. The vessel's performance for hydroacoustic survey is discussed from a viewpoint of noise level.

FRIDAY MORNING, 26 JUNE 1998

EAST BALLROOM B (S), 9:15 A.M. TO 12:15 P.M.

\title{
Session 5aBVa
}

\section{Bioresponse to Vibration/Biomedical Ultrasound and Physical Acoustics: Lithotripsy I}

\author{
Andrew J. Coleman, Cochair \\ Department of Medical Physics, St. Thomas Hospital, Lambeth Place Roth, London SE1 7EH, England \\ Robin O. Cleveland, Cochair \\ Department of Aerospace and Mechanical Engineering, Boston University, 110 Cummington Street, \\ Boston, Massachusetts 02215
}

Invited Papers

9:15

\begin{abstract}
5aBVa1. ESWL-The evaluation of a revolution. C. G. Chaussy (Staedt Krankenhaus München—Harlaching, München, Germany)

After 6 years of experimental research at the Departments of Urology and Surgical Research of the Ludwig-Maximilian University in Munich, extracorporeal shock-wave lithotripsy (ESWL) was introduced for clinical use in 1980. Uniquely successful and increasingly requested by stone patients, the method soon became widespread. Currently more than 2000 lithotriptors are in operation worldwide and over 5000000 treatments have been carried out successfully. Clinical experience in all centers has proven the safety, reliability, and reproducibility of the method. Currently approximately $70 \%$ of nonselected stone patients are eligible to receive ESWL treatment and, when combined with endourological procedures, more than $95 \%$ of patients can benefit from this method and thus avoid open surgery.
\end{abstract}

\section{9:35}

5aBVa2. Extracorporeal shock waves act by shock wave-gas bubble interaction. M. Delius (Inst. for Surgical Res., Univ. of Munich, Klinikum Grosshadern, 81366 Munich, Germany) and W. Eisenmenger (Institut für Physik 1, Univ. of Stuttgart, 70569 Stuttgart, Germany)

Previous animal experimental data had suggested that extracorporeal shock waves acted by the interaction of shock waves with remnant gas bubbles left from cavitational activity of previous shocks. In vitro experiments had shown that haemolysis from shock waves were reduced by $>95 \%$ by static express pressures from only $1-4 \times 10^{5} \mathrm{kPa}$ in the exposure vessel. This was interpreted as evidence for a reduced shock wave-gas bubble interaction by compression of the remnant gas bubbles by the excess pressure. 
Additional experiments have shown that haemolysis was lower at $0.2-1 \times 10^{5} \mathrm{kPa}$ excess pressure when the same number of shock waves was administered more slowly. Application of a single strong shock to red blood cells had little effect on haemolysis yet application of two shocks caused an increase of haemolysis by 500\%. Static excess pressure abolished the increase. The only interpretation one can think of is that the first shock had caused remnant gas bubbles which acted strongly at the second shock. The term shock-wave gas-bubble interaction is well known from cavitation physics and should be used more often to describe the mechanism of action of extracorporeal shock waves.

5aBVa3. Can SWL-induced cavitation and renal injury be separated from SWL-induced impairment of renal hemodynamics? Andrew P. Evan (Dept. of Anatomy, Indiana Univ. School of Medicine, MS 259, 635 Barnhill Dr., Indianapolis, IN 46202), Lynn R. Willis, Bret A. Connors, James A. McAteer, James E. Lingeman, Robin O. Cleveland, Michael R. Bailey, and Lawrence A. Crum (Univ. of Washington, Seattle, WA 98105)

SWL to one kidney causes localized tissue damage and impairment of tubular function, but reduces renal plasma flow (RPF) in both kidneys. We examined the effect of SWL voltage $(\mathrm{kV})$ and inversion of the waveform (IW) on these localized and bilateral effects of SWL. Five-week-old pigs were anesthetized for either sham-SWL or SWL (2000 shocks, unmodified HM3) at 12, 18, or 24 $\mathrm{kV}$ or 2000 shocks, $24 \mathrm{kV}$, with a reflector that inverts the waveform. $\mathrm{RPF}$ and tubular extraction of PAH $\left(\mathrm{E}_{\mathrm{PAH}}\right)$ were measured 1 $\mathrm{h}$ before and 1 and $4 \mathrm{~h}$ after SWL. $\mathrm{E}_{\mathrm{PAH}}$ estimates tubular secretion function. SWL significantly reduced RPF to similar degrees at each $\mathrm{kV}$. $\mathrm{E}_{\mathrm{PAH}}$ was not significantly reduced in the $12-\mathrm{kV}$ group, but was reduced to progressively greater degrees in the 18 and 24 $\mathrm{kV}$ groups. IW eliminated ultrasonic evidence of cavitation, produced minimal tissue damage, and eliminated the reduction of $\mathrm{E}_{\mathrm{PAH}}$. It did not eliminate the reduction of RPF. The data suggest that shock-wave voltage and cavitation may be related to the tissue injury and reduced $\mathrm{E}_{\mathrm{PAH}}$ induced by SWL, but suggests that neither may be directly related to the impairment of RPF. [Work supported by NIH, PO1 DK43881.]

\section{0:15-10:25 Break}

\section{$10: 25$}

5aBVa4. The potential of lithotripter shock waves for gene therapy of tumors. Douglas L. Miller, Richard A. Gies, Brian D. Thrall (P7-53, Battelle Pacific Northwest Natl. Lab., P.O. Box 999, Richland, WA, doug.miller@pnl.gov), and Shiping Bao (Washington State Univ., Richland, WA)

Tissue destruction by lithotripter shock-wave-induced cavitation can be effective for antitumor therapy. Since DNA transfection can be accomplished through cavitation-induced sonoporation, the potential may exist for an advantageous combination of shock wave and gene therapy of tumors. B16 mouse melanoma cells were cultured by standard methods and a luciferase reporter vector was used as the DNA plasmid. The shock-wave generation system, similar to a Dornier HM-3 lithotripter, had peak pressure amplitudes of 24.4 MPa positive and 5.2 MPa negative. In vitro exposures of cell suspensions indicated that results were greatly enhanced by leaving an air space in the exposure chambers to promote cavitation activity. For in vivo exposure, cells were implanted subcutaneously in C57BL/6 mice 10-14 days before treatment. DNA at $0.2 \mathrm{mg} / \mathrm{ml}$ and sometimes air at $10 \%$ of tumor volume was injected intratumorally before exposure. Exposure to 800 shock waves, followed by culture of isolated tumor cells for one day, yielded 1.1 $(0.43 \mathrm{SE}) \mathrm{pg}$ luciferase production per $10^{6}$ cells, increasing to $7.5(2.5 \mathrm{SE}) \mathrm{pg} / 10^{6}$ cells for air injection. Significant luciferase production occurred for 200,400, 800, and 1200 shock waves with air injection. Gene transfer therefore can be induced during lithotripter shock-wave treatment of tumors. [Work supported by NIH Grant No. CA42947.]

\section{0:45}

5aBVa5. Effects of lithotripter fields on biological tissues. Diane Dalecki (Dept. of Elec. Eng. and the Rochester Ctr. for Biomed. Ultrasound, Univ. of Rochester, Rochester, NY 14627)

Biological effects resulting from exposure to lithotripter fields include hemorrhage in soft tissues, such as the kidney, lung, and intestine, the production of premature cardiac contractions, malformations in the chicken embryo, and killing of Drosophila larvae. Pulsed ultrasound can produce similar bioeffects at comparable pressure thresholds. Tissues that contain gas bodies, either naturally or after the addition of ultrasound contrast agents, are particularly susceptible to damage from low-amplitude lithotripter fields. Lung and intestine contain gas naturally and are hemorrhaged by exposure to lithotripter fields on the order of $1 \mathrm{MPa}$. After the introduction of an ultrasound contrast agent into the vasculature, many organs and tissues, such as the bladder, kidney, fat, muscle, and mesentery, show extensive hemorrhage after exposure to lithotripter pressures less than $2 \mathrm{MPa}$. Tissues near developing bone are also selectively susceptible to damage from exposure to low-amplitude lithotripter fields. The thresholds for hemorrhage in tissues near developing bone, such as the fetal head, limbs, and ribs, are all less than $1 \mathrm{MPa}$ for exposures with a piezoelectric lithotripter. Cavitation and purely mechanical forces have been investigated as possible mechanisms for these biological effects of lithotripter fields.

\section{1:05}

5aBVa6. Biomechanical effects of ESWL shock waves. Bradford Sturtevant and Murtuza Lokhandwalla (Grad. Aeronautical Labs., Calif. Inst. of Tech., Pasadena, CA 91125)

Impulsive stress in the repeated shock waves administered in ESWL is the mechanical stimulus of injury to the kidney. In order to better understand the mechanical origins of injury, the interaction of focused shock waves with simple, planar polymeric membranes immersed in tissue-mimicking fluids was studied. The ESWL shocks in uniform, noncavitating liquids do not cause damage, but after passing through tissue or simulated tissue they do. This result suggests that the acoustic inhomogeneity of tissue may contribute to injury from ESWL. Shocks with large amplitude and short rise time (e.g., in uniform media) cause no damage in noncavitating fluids, while long-rise-time, dispersed shock waves, though only moderately attenuated, do. A continuum model is 
described which incorporates the mechanical properties of the tissue and accounts for the effect on its strength of microscopic inhomogeneities. It is shown that when transient tensile stress is applied it takes a finite time for failure to occur, so if the pulse is not long enough the material survives, in qualitative agreement with the behavior described above. A definition of dose at failure is derived in terms of the stress applied to the membrane by each shock wave, the strain rate, and the material failure stress.

\section{$11: 25$}

5aBVa7. Effects of tissue constraining on shock wave-induced bubble oscillation in vivo. Pei Zhong (Dept. of Mech. Eng. and Mat. Sci., Duke Univ., Box 90300, Durham, NC 27708)

Using a focused hydrophone (1 MHz), acoustic emission (AE) associated with the rapid oscillaton of cavitation bubbles induced by lithotripter shock waves was measured in kidney pelvis and renal parenchyma of a swine model, and compared with the $\mathrm{AE}$ produced in water under the same lithotripsy conditions. At each lithotripter output setting (between 16 and $24 \mathrm{kV}$ ), the duration of the primary bubble expansion/collapse was found to decrease as the AE was measured from water to kidney pelvis and renal parenchyma, respectively. As the number of shock waves delivered increased, the ratio of the primary bubble expansion/collapse to the subsequent ringing time was found to be almost unchanged in water, but varied significantly in both pelvis and renal parenchyma. These findings indicate a tissue-constraining effect on lithotripter shock wave-induced bubble oscillation in vivo. [Work supported by NIH.]

\section{Contributed Papers}

\section{1:45}

5aBVa8. SWL cavitation damage in vitro: Pressurization unmasks a differential response of foil targets and isolated cells. James A. McAteer, Mark A. Stonehill, Karin Colmenares, James C. Williams, Jr., Andrew P. Evan (Dept. of Anatomy, Indiana Univ. School of Medicine, 635 Barnhill Dr., Indianapolis, IN 46202-5120, mcateer@anatomy.iupui.edu), Robin O. Cleveland (Boston Univ., Boston, MA 02115), Michael R. Bailey, and Lawrence A. Crum (Univ. of Washington, Seattle, WA 98105)

To better understand the role of cavitation in kidney stone comminution and renal injury pressurization (Delius UMB23:1997) to regulate cavitation bubble activity in vitro was used. A low acoustic impedance chamber $(8 \times 15 \mathrm{~cm})$ that only minimally altered lithotripter shock-wave pressure and waveform was constructed to house targets (aluminum foils or vials of kidney epithelial cells) under pressure (0-1400 psi). Cells exhibited lytic injury and foils sustained pitting at atmospheric pressure, but high pressure (1400 psi) prevented lysis and pitting. The pressure threshold for reduced damage was dramatically different for cells versus foils. Cell lysis was prevented at very low pressure $(>30 \mathrm{psi})$, while substantially greater pressure $(\sim 600 \mathrm{psi})$ was needed to prevent pitting. Modest pressure (25-300 psi) actually enhanced pitting. SWL in vitro cell lysis and foil pitting are both likely due to cavitation. However, interactions between cavitation bubbles and these two targets appear to be quite different. Our observations are consistent with those of Delius and support his suggestion that it may be possible to reduce cavitation-mediated cell damage by regulating ambient pressure. The finding that slight pressure increased the number of cavitation events on foils suggests that modest overpressure might enhance stone comminution. [Supported by NIH PO1DK43881.]

\section{2:00}

5aBVa9. Effect of overpressure on dissolution and cavitation of bubbles stabilized on a metal surface. Robin O. Cleveland (Dept. of Aerosp. and Mech. Eng., Boston Univ., Boston, MA 02215), Michael R. Bailey, Lawrence A. Crum (Appl. Phys. Lab., Univ. of Washington, Seattle, WA 98105), Mark A. Stonehill, James C. Williams, Jr., and James A. McAteer (Indiana Univ. School of Medicine, Indianapolis, IN 46202-5120)

Recent experimental evidence indicates that static overpressure dramatically reduces lithotripsy-induced cell damage or stone comminution [Delius, Ultrasound Med. Biol. 24, 611-617]. The hypothesis is that the damage and comminution are due to cavitation and that overpressure dissolves the small gas bubbles which act as cavitation nuclei. Delius observed, however, that the overpressure to protect cells (1 atm) was significantly lower than for stones (10 atm). In similar experiments cell lysis and pitting of aluminum foil as indicators of cavitation are used; the thresholds were 1 and $30 \mathrm{~atm}$, respectively. In addition, the authors observed that pitting of foils increased for overpressures up to $20 \mathrm{~atm}$. It is proposed that crevices in the foil stabilize cavitation nuclei whereas the bubbles dissolve in fluids. Foil damage increased because overpressure is small relative to the shock wave pressures that drive bubble expansion but is the dominant driving force at bubble collapse. Calculations using the Gilmore equation predict that overpressures up to $30 \mathrm{~atm}$ hasten and intensify cavitation collapses and over $60 \mathrm{~atm}$ eliminate cavitation, in qualitative agreement with our foil data. The calculated collapse time was confirmed experimentally by passive cavitation detection. If bubbles are stabilized in crevices in kidney stones, overpressure may provide a safer, more effective lithotripsy treatment. [Work supported by NIH-PO1-DK43881.] 


\title{
Session 5aBVb
}

\section{Bioresponse to Vibration/Biomedical Ultrasound: Medical Ultrasound II-Propagation, Media Characterization and Miscellaneous Topics}

\author{
Timothy G. Leighton, Chair \\ Institute for Sound and Vibration Research, University of Southampton, Highfield, Southampton SO17 1BJ, England
}

\section{Contributed Papers}

\section{9:15}

5aBVb1. Are blood clots Biot solids? Pierre D. Mourad and Steven G. Kargl (Appl. Phys. Lab., UW, 1013 NE 40th St., Seattle, WA 98105, pierre@apl.washington.edu)

Blood clots are saturated porous solids. Their unwanted presence in veins and arteries is the source of a variety of medical problems. Their destruction (thrombolysis) via ultrasound and various enzymes (ultrasound-enhanced thrombolysis, or UET) is still a poorly understood phenomenon. The current view is that cavitation is the mechanism behind UET. This presentation begins with a review of the structure of blood clots and natural thrombolysis. Following that is a review of the literature on UET pointing out that cavitation is at best part of the story, at least in vitro, and more suspect in vivo. Next is a motivation of the Biot theory (a theory for how sound propagates through and interacts with porous solids) and its application to blood clots. The results of the work thus far predict that blood clots are Biot solids, which has implications for understanding UET. [Work sponsored by DARPA.]

\section{9:30}

5aBVb2. A stratified model for ultrasonic propagation in cancellous bone. Elinor R. Hubbuck, Timothy G. Leighton, Paul R. White (Inst. of Sound and Vib. Res., Univ. of Southampton, Southampton SO17 3BJ, Great Britain), and Graham W. Petley (Southampton Univ. Hospitals NHS Trust, Southampton, Great Britain)

A new model for ultrasonic wave propagation in cancellous bone is presented here. The model treats cancellous bone as a stratified medium of periodically alternating solid-fluid layers, and considers propagation of two compressional modes, analogous to the fast and slow waves of Biot theory, in terms of slowness surfaces. The behavior of the two modes is examined in vitro using bovine bone samples over a range of incidence angles. Two modes are seen when the cancellous structure is parallel to the propagation direction, but only one mode propagates normal to the structure, and the velocity of the fast wave increases with angle of incidence away from the normal. This behavior can be explained in terms of an angular dependent inertial coupling effect, in keeping with the theory of propagation in stratified media of periodically alternating fluid-solid layers. Quantitative agreement with theory is good. Although the structure being analyzed is essentially an oversimplification of the structure of cancellous bone, the agreement with theory suggests the stratified model offers potential for future research.

\section{9:45}

$5 \mathrm{aBVb3}$. Effects of the structural anisotropy and the porosity on ultrasonic wave propagation in bovine cancellous bone. Takahiko Otani and Atsushi Hosokawa (Dept. of Elec. Eng., Doshisha Univ., 610-0321 Kyotanabe-shi, Japan)

Ultrasonic wave propagation in water-saturated bovine cancellous (spongy) bone has been experimentally studied in vitro by a pulse transmission technique. Fast and slow longitudinal waves have been clearly observed in the earlier investigation when the acoustic wave propagates to the direction of the trabeculae orientation [A. Hosokawa and T. Otani, J.
Acoust. Soc. Am. 101, 558-562 (1997)]. In the present study, propagation speeds of the fast and the slow waves were measured as a function of the propagation angle to the trabeculae orientation. Experimental results show that the propagation speed of the fast wave and the amplitude of the slow wave depend greatly on the angle to the trabeculae orientation, and the speed of the slow wave and the amplitude of the fast wave are little affected by the angle of the trabeculae orientation. The propagation speeds of both waves were also experimentally examined as a function of the porosity. Measured results are discussed in relation to the structural anisotropy and the porosity using Biot's theory.

10:00

5aBVb4. Wideband laser-acoustic spectroscopy of proteins. Alexander A. Karabutov and Natalia B. Podymova (Dept. of Phys., Moscow State Univ., Moscow, Russia 119899,

karabut@gpwp1.phys.msu.su)

The absorption of ultrasound in a frequency range of $2-50 \mathrm{MHz}$ in proteins was investigated with a wideband laser-ultrasonic spectrometer. It was found that for fresh chicken proteins ultrasonic absorption is proportional to the squared frequency. Denaturation of proteins transforms the spectrum of ultrasonic absorption to the first power of frequency dependence. In the hole frequency range the ultrasonic absorption in denaturated protein is higher than in the fresh one. The possibility of laser ultrasonic diagnostics of proteins is discussed.

\section{0:15}

5aBVb5. Wideband acoustics spectroscopy of liquid phantoms of biological tissues. Valery G. Andreev, Yury A. Pischalnikov (Dept. of Acoust., Phys. Faculty, Moscow State Univ., Moscow 119899, Russia, andreev@na.phys.msu.su), Alexander A. Karabutov, and Natal'ya B. Podymova (Moscow State Univ., Moscow 119899, Russia)

Biological tissues have a specific frequency dependence of the ultrasonic absorption and sound-speed dispersion. It makes fruitful the diagnostics of tissues by studying these parameters. It is necessary to carry out the spectroscopic investigation in a wide frequency band in a real time. Pulsed laser ultrasonic spectroscopy is the most useful technique to solve this problem. The method of wideband acoustic spectroscopy with laser thermo-optical source is proposed. A Q-switched YAG-Nd laser was explored to excite the short acoustic transients (pulse duration $12 \mathrm{ns,} \mathrm{ampli-}$ tude up to $8 \mathrm{MPa}$ ) by an especially produced thermo-optical generator. The acoustic transients transmitted through the medium under the testing were detected by a wideband transducer (frequency band $2-350 \mathrm{MHz}$ ). The absorption of ultrasound was calculated by FFT. The absorption in glycerol, acetic acid, and butanediol was investigated in a frequency band 2-50 MHz. The spectra of relaxation times were calculated. [Work supported by RFBR and CRDF.] 
5aBVb6. The necessity for acoustics in the biomedical engineering program. Daniel R. Raichel (Dept. of Mech. Eng., Steinman Hall, The City College of The City Univ. of New York, New York, NY 10031) and Latif Jiji (The City College of CUNY, New York, NY 10031)

Biomedical engineering constitutes the fastest growing segment of all engineering fields. In order to effectively deal with a wide variety of engineering problems, the biomedical engineer needs to be a "jack of all trades" insofar that he or she should be able to meet challenging problems in mechanics, electronics, thermal analyses, fluid mechanics, materials, manufacturing techniques, etc. In the medical field, acoustics (particularly ultrasound) is used for both diagnostic and therapeutic purposes, and new aging or more effective therapy. As a part of the biomedical engineering program, undergraduate and graduate students need to gain better understanding of acoustical physics, the effects of sound and ultrasound on living organisms, the principles of measurements, imaging processes, and data analysis concepts. Laboratory experience is essential, and practical experience in a teaching medical hospital would definitely be salutary. The time constraint imposed by a four-year undergraduate program, which also needs to meet ABET criteria, effectively limits the expository scope, and it follows that additional, more intensive training may be better achieved through post-baccalaureate (master's degree and doctoral levels) programs.

FRIDAY MORNING, 26 JUNE 1998

EAST BALLROOM A (S), 9:15 TO 11:30 A.M.

\title{
Session 5aEA
}

\section{Engineering Acoustics: Sonar Transducers}

\author{
James M. Powers, Chair \\ Naval Undersea Warfare Center, Code 2131, Newport, Rhode Island 02841-1708
}

\section{Contributed Papers}

\section{$9: 15$}

5aEA1. Finite-element simulation of piezoelectric transformers. Takao Tsuchiya, Yukio Kagawa, and Hiroki Okamura (Dept. of Elec. and Electron. Eng., Okayama Univ., Okayama, 700 Japan)

A three-dimensional finite-element approach to the characteristic prediction of piezoelectric transformers is presented. For a numerical example, a Rosen-type piezoelectric transformer is considered. The electrical input admittance and the transmission or transfer characteristics are demonstrated for the fundamental longitudinal mode operation. Based on these characteristics, the lumped parameters are extracted to form the electrical equivalent circuit. The effect of the loading or termination condition on the output voltage and the conversion efficiency are also examined. The numerical results are then compared with the experimental results. Examination is then extended to the optimum design in terms of efficiency and the output voltage.

\section{9:30}

5aEA2. A tubular piezoelectric actuator and its characteristic analysis by finite-element modeling. Naoto Wakatsuki, Takao Tsuchiya, Yukio Kagawa, and Kazumichi Hatta (Dept. of Elec. and Electron. Eng., Okayama Univ., Okayama, 700 Japan)

Three-dimensional motion is sometimes required in an actuator. A tubular configuration is proposed to realize the three-dimensional motion at the tip. A cylindrical shell piezoceramic, polarized in the radial direction, is provided with several pairs of electrodes on both surfaces of the shell. The combination of the voltages with proper sense makes it possible to control the motion. The displacements or the forces at the tip are expressed in terms of the linear combination of the electrical input voltages on each pair of electrodes. The coefficient of each term is to be determined by the experiment or numerical simulation. Here, the determination of the coefficients is made by finite-element modeling for which the code previously developed is utilized.
9:45

5aEA3. Substructuring in the finite-element analysis of sonar transducer arrays. J. R. Dunn (School of Electron. \& Elec. Eng., Univ. of Birmingham, Edgbaston, Birmingham B15 2TT, England, j.r.dunn@bham.ac.uk), C-L. Chen (6F-3, Taipei, Taiwan), and B. V. Smith (Univ. of Birmingham, Edgbaston, Birmingham B15 2TT, England)

Finite-element (FE) analysis of arrays of sonar transducers becomes costly in memory and computation time for arrays with many elements. This becomes even more severe a problem if the individual transducers have complex structures. However, because in many cases the individual transducer elements are identical, it is possible to treat them as repeated substructures; each substructure may then be viewed as a superelement which is defined by only those nodes which couple into the overall structure of the array. This technique is analogous to the use of Thevenin equivalent sources in the analysis of complex electrical circuits. By the use of this technique it is possible to analyze the electroacoustic performance of larger arrays than hitherto possible within the limitations set by the computer memory. This paper describes this superelement technique and results are presented for a simple two-element transducer array analyzed for its free (unloaded) vibration behavior both by full $\mathrm{FE}$ analysis of the complete structure and by the substructuring technique. The results of the two methods are shown to agree very closely, and measurements on an experimental model compare favorably with the theoretical predictions.

\section{0:00}

5aEA4. Sonar simulation and display techniques. Yuhong Guo, Hong Liu, and Junying Hui (Dept. of Underwater Acoust. Eng., Harbin Eng. Univ., 150001 Harbin, PROC, guoyh@public.hr.hl.cn)

This paper describes techniques used in the simulation and display of a passive sonar system. The sonar and its environment models, including target, channel, noise background, and array, and a complete sonar signal processing chain are developed in the simulation system. Based upon the simulation system, different display techniques are studied. Modeling for performance assessment of a colored brightness mode sonar display is difficult because of its relation to human visual psychology and physiology. A concept of "visual threshold" is proposed in the paper; based on it, as well as the statistical model of display data, detection performance of 
the sonar display system can be analyzed quantitatively and theoretically. The second-decision method, based on the statistical property of LOFAR display, and a four-dimensional display technique are presented in the paper.

\section{0:15-10:30 Break}

\section{0:30}

5aEA5. A new method of multibeamforming for a swath bathymetry sonar system and its MIMD parallel architecture. Haisen $\mathrm{Li}$, Lan Yao, and Xinsheng Xu (Dept. of Underwater Acoust. Eng., Harbin Eng. Univ., Harbin 150001, PROC, euipc@ public.hr.hl.cn)

In a swath bathymetry sonar system, real-time multibeamforming is required in order to increase the coverage and the efficiency. To determine the direction of seafloor echo accurately, traditional beamforming must work at a high sample rate, the interpolation is used to reduce computing amounts, but when there are tens of receiving channels and beams, the interpolation filter algorithm will rapidly increase computing amounts to result in the real-time multibeamforming, this is very difficult. Combining with a swath bathymetry sonar system, a new method of quadrature beamforming is described in this paper. It combines time domain beamforming with phase shifting beamforming to process the available bandpass signal. To reduce sample rate, a mixing frequency technique is used. A quadrature component is obtained from a single-channel A/D converter utilizing Hilbert transformation and its coefficient is weighted with Hamming window function, therefore, amplitude and phase is highly accurate. Comparing with the traditional beamforming method, the method presented in the paper is characterized by high accuracy, less sample rate, and less computation complexity, easily realized with a high speed DSP device. The parallel pipeline architecture is given based on TMS320C30. Simulating and lake trial results show that the method is right and efficient. The method is universal for active sonar.

\section{0:45}

5aEA6. Specific acoustic impedance of the ultrasonic field by the square flat transducers. Tohru Imamura (Natl. Res. Lab. Metrology, 1-1-4, Umezono, Tsukuba, Ibaraki, 305 Japan, imam@nrlm.go.jp)

Sound pressure, particle velocity, and specific acoustic impedance are investigated, using the case where square flat transducers are tilted from a parallel condition. Specific acoustic impedance is a quotient of sound pressure by particle velocity. Particle velocity is computed by numerical quadruple integration with Huygens's principle, and compared with that of the plane wave. The ratio of the half side length (a) of the square flat transducer to the wavelength $(\lambda)$ of the ultrasonic wave is set at 2.5 , and the distance between the transducers is set at $5 \mathrm{a}$ or 10a. The feature of the phase delay leap of the specific acoustic impedance on the central axis of the circular flat transducer does not appear for the square case.
11:00

5aEA7. Estimation of equivalent circuit parameters for piezoelectric transducer using least-squares method. Byung-Doo Jun (R \& D 3 Gr., LG Precision Co. Ltd., 148-1 Mabuk-ri, Gusung-Myun, Yongin-shi Kyunggi-do, 449-910, Korea) and Koeng-Mo Sung (Seoul Natl. Univ., Seoul 151-742, Korea)

In general, the resonance method has been used for estimating the equivalent circuit parameters for the underwater piezoelectric transducer. Sometimes, however, it is difficult to estimate the equivalent circuit parameters when the measured electrical impedances have an imaginary part at resonant and antiresonant frequency. In this paper, to estimate the equivalent circuit parameters of the piezoelectric transducers, equivalent circuit equation is modified and the least-squares method is applied. Equivalent circuit equation is composed of real and imaginary equations, respectively. With these equations, matrix form Yule-Walker equations are formulated. The least-squares method is applied with measured electrical impedance data over the effective frequency band to solve these equations. The procedure is as follows: First, four variables which are functions of five equivalent circuit parameters $(\mathrm{R}, \mathrm{L}, \mathrm{C})$ are obtained by the least-squares method. Then, initial equivalent circuit parameters can be extracted by solving four equations which contain five parameters, one of which is assumed. Finally, by iteration and update, all the equivalent circuit parameters can be estimated with considerably small error. The impedance characteristics of the piezoelectric transducer with estimated parameters are in good agreement with measured ones.

\section{1:15}

5aEA8. Study of compound structured ultrasonic transducer made of PZT/PVDF. Moo Joon Kim, Dong Hyun Kim, and Kang Lyeol Ha (Dept. of Phys., Pukyong Natl. Univ., 599-1 Daeyon-dong, Nam-gu, Pusan 608-737, Korea)

When the piezoelectric ceramic, PZT is used in the water it needs matching layers for efficient energy transmission. PZT is known as the proper piezoelectric material for the transmitter by reason of the large electromechanical coupling factor. So actually PZT has been used for the nondestructive testing or ultrasonic diagnostic instrument as good material. In the meantime, there is a kind of polymer by the name of PVDF. Its acoustic impedence is similar to water, so PVDF does not need matching layers but it is not a proper material for the transmitter because of the small electromechanical coupling factor. However, the receiving sensitivity of PVDF is very flat through the wide frequency range. So it is known as a proper material for the receiver like wideband hydrophone. Therefore in this paper it is suggested that the transducer which transmits and receives signals by PZT and PVDF, respectively, could be constructed compositively, and the transmitting and receiving feature of the transducer was investigated theoretically and experimentally. 


\title{
Session 5aMU
}

\author{
Musical Acoustics: Pastiche \\ Vladimir Chaloupka, Chair \\ Physics Department, University of Washington, Box 351560, Seattle, Washington 98125-1560
}

\section{Contributed Papers}

\section{9:00}

5aMU1. Physics of the Music Laboratory at the University of Washington. Vladimir Chaloupka and Keith Hughes (Phys. Dept. 351560, Univ. of Washington, Seattle, WA 98125-1560)

The Physics Department at the University of Washington recently completed the acquisition of new equipment for teaching the Physics of Music course, as well as for performing various independent study projects in musical acoustics at both undergraduate and graduate levels. The lab setup will be described, as well as the experience after the first year of operation. Many aspects of the Physics of Music provide excellent opportunities for involving undergraduate students in small, but meaningful and interesting, research projects. Specific examples will be discussed.

\section{9:15}

5aMU2. Dynamic optimal tuning of electronic keyboards as they are being played. James E. Steck (Dept. of Mech. Eng., Wichita State Univ., Wichita, KS 67260-0133, steck@me.twsu.edu) and Dean K. Roush (Wichita State Univ., Wichita, KS 67260)

The 12-tone equally tempered musical scale can produce noticeably imperfect matching of acoustic harmonics when some combinations of tones are played together. The lower partials (harmonics) which should match, do not. For a 12-tone octave, if a small number of tones in the octave are to be sounded simultaneously, a tuning can be found which produces harmonic errors that are much less than that of equal temperament. Historically, these tunings have been discovered, explored, and abandoned in favor of equal temperament because they generally have much worse harmonic error for tone combinations other than those for which the tuning was optimized. Here, a mathematical description of the total harmonic error of a musical instrument is presented along with a mathematical method which solves for a tuning (tone frequencies) that minimize this total error. The optimal tuning calculations have been implemented in " $\mathrm{C}$ " code on a 166-MHZ pentium desktop personal computer equipped with a Creative Labs Sound Blaster AWE 32 soundcard, with a MIDI keyboard attached. The " $\mathrm{C}$ " code reads the keys (tones) being played on the keyboard, calculates the optimal tuning, and sounds the tones on the PC soundcard at the optimally tuned fundamental frequencies.

\section{9:30}

5aMU3. On the use of elements of piano notes to improve the identification of polyphonic piano sounds. Lucile Rossi and Gerard Girolami (Univ. of Corsica, Quartier Grosseti, 20250 Corte, France, lrossi@univ-corse.fr)

An original algorithm for identifying polyphonic piano sounds signals, based only on the use of frequential positions of note partials, has been developed by the authors [Rossi and Girolami, J. Acoust. Soc. Am. 100, 2842(A) (1996)]. This paper presents a study of the evolution of the amplitude of piano notes' partials and their energy, carried out to see if these elements can be used to improve the identification of notes in cases like short notes, long notes, repeated notes, and polyphonic sounds. In the case of monophonic sounds, Doval [Ph.D. thesis, University of Paris VI (1994)] has shown that the evolution of the amplitude of piano notes' partials along with their frequency variations can be used to identify the fundamental frequency of signals. The case of polyphonic piano sounds (several notes played simultaneously on the same piano) is more difficult, but if a particular behavior of partials belonging to a given note could be detected, for example, synchronous variations or typical amplitude evolution, the sorting of partials would be more reliable, the identification of notes more efficient, and the detection of notes' onsets more precise.

\section{$9: 45$}

5aMU4. A new accurate model-based music synthesis technique by using recurrent neural networks. S. F. Liang (Dept. of Control Eng., Natl. Chiao-Tung Univ., Hsin-Chu, Taiwan) and Alvin W. Y. Su (Dept. of CSIE, Chung-Hwa Univ., Hsin-Chu, Taiwan)

Reproduction of the tones generated by playing a particular musical instrument electronically remains an open and difficult problem. An accurate model-based analysis/synthesis approach with respect to pluckedstring instruments by using recurrent neural networks is proposed. The procedure is described as follows. A recurrent neural network corresponding to the physical model of a musical string is first configured. The vibration of a plucked string is measured and used as the training data of the neural network. The backpropagation-through-time method is used to train the network such that output of this network can be matched to the measured data as close as possible. The well-trained neural network is then used as our synthesis model for this particular string. Since the characteristics of a string keep changing over the period of vibration, it is also necessary to update the parameters of the neural network during the synthesis process. In our experiments the synthesized tones and the real tones sounded almost identical. These results will also be demonstrated during the presentation in ICA/ASA 98 if this paper is accepted. [This Work is supported by both NSF, Taiwan and Computer/Communication Lab. ITRI, Taiwan.]

\section{0:00}

5aMU5. Musical instrument synthesis by nonregenerative nonlinear processing. James W. Beauchamp (School of Music and Dept. of Elec. and Comput. Eng., Univ. of Illinois at Urbana-Champaign, Urbana, IL 61801)

When a sinusoid is distorted by a nonlinear function, a complex tone is produced. An $n$th degree polynomial nonlinear function produces an output waveform containing $n$ harmonics. Moreover, when the amplitude of the input sine wave changes, the harmonic structure of the output changes in a way which can be predicted from the polynomial coefficients. Also, the coefficients can be set to provide any arbitrary magnitude spectrum when the sine wave has a particular amplitude, say unity. Generally, the spectral centroid, a measure of the spectral bandwidth, increases monotonically as the input amplitude increases. This possibility is enhanced by placing a high-pass filter at the output. An acoustic instrument sound can be imitated by (1) performing time-variant harmonic analysis on it, (2) selecting a representative spectrum from it to determine the polynomial coefficients, (3) measuring the sound's time-varying spectral centroid and rms amplitude, and (4) coercing the sine wave amplitude to change so that the spectral centroid of the output matches that of the original sound. The amplitude of the original sound is matched by a multiplier at the nonlinear output. This method has been successfully used to synthesize instruments such as the trumpet, saxophone, clarinet, oboe, and piano. 
5aMU6. Time-domain modeling and numerical simulation of timpani. Leila Rhaouti, Patrick Joly (Inria-Rocquencourt, BP 105, 78153 Le Chesnay Cedex, France), and Antoine Chaigne (Enst, Paris Cedex 13, France)

Timpani are made of a circular elastic membrane stretched over a enclosed air cavity and set into vibrations by the impact of a mallet. The motion of the membrane is coupled with both the external and internal sound pressure field. A time-domain model of this instrument has been developed in order to investigate the influence of the main geometrical and physical quantities on the resulting sound. This model consists of a set of partial differential equations which govern the displacement of the membrane and the acoustic pressure inside and outside the cavity, respectively. These equations are coupled with a nonlinear differential equation which governs the excitation by the mallet. A numerical scheme has been derived from this model using three-dimensional finite element methods. Absorbing conditions have been implemented to simulate the free space. The validity of the model is illustrated by successive snapshots showing both the pressure field and membrane displacement. In addition, time histories of energetic quantities are presented for a better understanding of energy balance between membrane, cavity, and external space in real instruments. Sound examples are obtained by simulating the sound pressure at specific positions corresponding to the player's ears.
5aMU7. Modeling Chinese musical instruments. Andrew B. Horner and Lydia Ayers (Dept. of Comput. Sci., Hong Kong Univ. of Sci. and Technol., Clear Water Bay, Kowloon, Hong Kong, horner@cs.ust.hk)

Chinese instruments include a range of colorful instruments with distinctive characters. For example, the dizi is the most common Chinese flute, and it has a bright buzzing quality produced by a rice paper membrane glued over a hole. This paper describes models for more than 20 Chinese traditional and folk instruments using group additive synthesis with genetic algorithm-optimized parameters. Other types of Chinese flutes modeled include the xiao (vertical flute), paixiao (panpipes), and xun (ocarina). The sheng is a mouth organ with a ring of bamboo pipes attached to a wind chamber, and dates back to $1500 \mathrm{BC}$. A folk version of the sheng, called the lusheng, has also been modeled, as well as the bawu and hulusi, folk instruments that sound similar to the clarinet. The player's mouth completely covers the bawu's blowhole, which has a vibrating reed cut into a copper strip covering the hole. The hulusi has a playing tube and multiple drone tubes. Several pitched percussion and plucked string instruments have also been modeled. The presentation will show the acoustic instruments, their sound, their spectra, and resynthesized excerpts of music written for these instruments.
FRIDAY MORNING, 26 JUNE 1998

CASCADE BALLROOM I, SECTION A (W), 9:00 TO 10:15 A.M.

\title{
Noise: Aircraft Noise: General
}

\author{
Colin G. Gordon, Cochair \\ Colin G. Gordon and Associates, 411 Borel Avenue, Suite 425, San Mateo, California 94402 \\ Mei Q. Wu, Cochair \\ Colin G. Gordon and Associates, 411 Borel Avenue, Suite 425, San Mateo, California 94402
}

\section{Contributed Papers}

9:00

5aNSa1. Low-frequency noise around airports. Michel Vallet and Jean Claude Bruyere (INRETS, 25 avenue Francois Mitterrand, Case 24 69675, Bron Cedex, France)

The sound frequency weighting PNdB has been proposed by Kryter (1959) to assess the noise from jet engine aircrafts. Due to the technical evolution of aircraft engines, and to choose a frequency weighting common to all transport systems, the $\mathrm{dB}$ (A-weighted) has begun to be used as the acoustical unit around airports; the PNdB is still used for noise certification purposes. A study of noise measurements that was carried out around French airports allows one to see some noticeable differences between acoustical levels, respectively, expressed in $\mathrm{dB}(\mathrm{A})$ and $\mathrm{dB}(\mathrm{C})$. The difference varies between $4 \mathrm{~dB}$ for the landing of recent aircrafts to $14 \mathrm{~dB}$ at take-off for 50/70-seat propeller aircrafts. If one considers the additional frequency decrease due to the walls and windows in housing, the pertinence of $\mathrm{dB}(\mathrm{A})$ weighting could be questioned for aircraft noise received by people living a long distance from the airport. This question is of interest in light of the new ANSI method for assessing combined noises (Schomer, 1997): this method suggests using the $\mathrm{C}$ frequency weighting for sounds with strong low-frequency content.
9:15

5aNSa2. Subjective response to aircraft flyover noise. Sherilyn A. Brown (Structural Acoust. Branch, NASA Langley Res. Ctr., M.S. 463, Hampton, VA 23681, s.a.brown@larc.nasa.gov) and R. David Hilliard (Wyle Labs., Hampton, VA 23666)

In order to obtain increased understanding of community response to aircraft noise, a comprehensive subjective study was designed and implemented to realistically simulate the aircraft noise exposure experienced by residents living both near and far from airports. The test was designed to present a wide range of daily aircraft noise exposures ranging broadly in both quantity and amplitude. The In-Home study was supported by a computer controlled sound playback system which exposed test subjects to aircraft sounds during a daily $14 \mathrm{~h}$ test period. This system provided a degree of control over the noise exposure not found in community situations and a degree of situational realism not available in the laboratory. Each day the system played from 0 to 448 flyovers as the test subject went about his or her normal activities. At the end of the day, the test subjects rated their annoyance to the flyovers they had heard. The noise exposures presented during 9 weeks simulated different combinations of runway activity, aircraft flyover altitude, rush hour conditions, and occasional loud aircraft among many low sound level flights. Subsequent data analysis will 
seek to provide a basis of understanding of annoyance response to enhance predictive algorithms for a wide range of aircraft noise exposure conditions.

\section{9:30}

5aNSa3. Low-flying military aircraft noise-Operational flying. Ian H. Flindell (Inst. of Sound and Vib. Res., Univ. of Southampton, UK) and Ralph J. Weston (Royal Air Force Inst. of Health, Aylesbury, Buckinghamshire, UK)

The distribution of military low-flying training across Great Britain is essentially random within the permitted low-flying areas, although there are certain areas where above-average concentrations of flights occur. However, there are very little data available on actual flight tracks as flown, and training operations are normally conducted so as to encourage as much flexibility as possible. This means that calculations of noise exposure in different areas have to be based to a considerable extent on assumptions about typical flight track distributions, using aircraft noise source reference levels extrapolated from data collected under specially arranged measurement trials. This paper reports the results of a number of recent field surveys carried out in representative overflown areas which show generally good agreement between the assumptions made about typical flight distributions, the special trials data, and actual operational flying.

\section{9:45}

5aNSa4. Converting noise level into number of noisy events: A new method to quantify $k$ factors reveals area-dependent over- and underenergetic responses around the same airport. Karl Th. Kalveram (Univ. of Duesseldorf, Dept. of Cybernetical Psych., 40225 Duesseldorf, Germany, kalveram@uni-duesseldorf.de)

For aircraft noise, caused by $N$ flight movements within observation time $T$, with average maximum noise level $L_{\max }$ and duration $d_{t}$, the energy equivalent noise level $L_{\text {eq3 }}$ is approximated by $Q(k, d)=L_{\max }+k^{*} \log N+d^{*} \log (d t / T)$, if $k=d=10$. This seems to enable a trade-off between $L_{\max }, N$, and $d t$. However, the problem is not to keep the $L_{\text {eq3 }}$ constant, but the subjective annoyance, and this in turn depends on whether people process alterations of $L_{\max }, N$, and $d t$ corresponding to energy equivalence $(k=d=10)$ or not. Therefore, valid measurements of public noise responses by $Q(k, d)$ require the estimation of $k$ and $d$ from empirical data. However, multiple correlation techniques mostly used for this purpose lead to contradictory and invalid estimates for $k$. This paper presents an alternative method, utilizing dose-response curves based on regression of annoyance with $L_{\max }$ at fixed numbers of overflights. Results are independent from the selected annoyance ratings and furthermore also valid for nonlinear dose-response curves. Based on curves characterizing small $(N<5000)$, medium $(50000<N<200000)$, and large $(200000$ $<N)$ airports [Rice (1980)], the proposed procedure predicts a crossover effect also for different areas of the same airport, i.e., $k>10$ for low $k$ and $k<10$ for high noise levels, if this airport changes from medium to large.

10:00

5aNSa5. Relational anomalies in $\mathbf{A}$ weighted and linear scale sound level measurements associated with jet aircraft departures. Errol Nelson P.E. QEP (Optimum Environment, P.O. Box 114, Issaquah, WA 98027) and Allan Furney (Regional Commission on Airport Affairs, Des Moines, WA 98198)

Noise levels from jet aircraft operations at major airports are often perceived by nearby residents as being louder and more intrusive than claimed by airport proprietors. This can cause heated opposition by citizens to airport development and expansion plans. To further clarify the issue, a noise study was conducted using four noise meters; two Type 1 and two Type 2. Simultaneous sound level measurements were taken on the A-weighted and linear scales in airport and nonairport noise environments; and included octave band analyses. In both noise environments the average differential between full spectrum A-weighted and linear scale measurements is approximately $15 \mathrm{~dB}$, except when influenced by jet aircraft departures. During jet aircraft departures, this A/linear differential was as small as $4 \mathrm{~dB}$; and the smaller the difference between simultaneous measurements the louder the perceived noise from the departing plane. This measurement anomaly was unique to departing jet aircraft. Propeller driven aircraft operations, or jet aircraft landings, showed little or no change in the A/linear noise differential. The measurements suggest that a significant amount of noise is not being measured by the sound level meters during jet aircraft departures: either deducted by weighting filters or due to instrument limitations. However, by conducting simultaneous measurements of A weighted and linear scale sound levels, this relational anomaly can provide a simple, realistic indicator of jet aircraft loudness in the airport noise environment.
FRIDAY MORNING, 26 JUNE 1998

CASCADE BALLROOM I, SECTION C (W), 8:30 TO 9:30 A.M.

\title{
Noise: Source Noise Analysis and Control
}

\author{
Daniel A. Quinlan, Chair \\ Lucent Technologies Acoustics Research, 600 Mountain Avenue, Murray Hill, New Jersey 07974
}

\section{Contributed Papers}

8:30

5aNSb1. The use of frequency-dependent velocity scaling as a diagnostic tool. Daniel Quinlan (Bell Labs., Lucent Technologies, 600 Mountain Ave., Murray Hill, NJ 07974, dbq@lucent.com)

This paper will discuss a method for investigating broadband aeroacoustic noise sources. The method is based upon the use of frequencydependent velocity scaling. The dependence of sound power level upon a characteristic velocity is determined experimentally, and that dependence is used as an indicator of primary aeroacoustic processes. The procedure yields an identification of distinct frequency bands within which source trends are observable. Each band is presumed to be controlled by different processes, and the set of likely processes is fixed according to the average velocity exponent value obtained. In principal, the method is applicable to low-speed aeroacoustic source identification problems where a characteristic flow speed can be measured and systematically varied. Measurements of sound radiated by flow over a flat plate were used to evaluate the performance of the method. The procedure was then applied to a small axial fan typical of those used to cool electronic systems. For the fan, the frequency-dependent velocity scaling demonstrated that shifts in dominant aeroacoustic processes occur near $2 \mathrm{kHz}$ and above $4 \mathrm{kHz}$. The results added insight to a related study which demonstrated that tip gap flows were the primary source of broadband noise above $2 \mathrm{kHz}$. 
sively been performed on the generation of noise in premixed methane or propane flames. Syngas fuel is a mixture of hydrogen and carbon monoxide, and the burners used are of the non-premixed type. In this research, the effect of turbulence and syngas composition on noise generation is investigated. A laboratory is set up to test syngas flames of a thermal power of $50 \mathrm{~kW}$ in a cylindrical air-cooled combustion chamber. Experiments are performed at several fuel compositions and burner inlet conditions. The flame sound intensity is measured in the combustion chamber equipped with acoustic dampers. The paper discusses the measured sound spectra. A model is derived for the generation of sound in a turbulent non-premixed flame. In this model it is shown that the sound generation is related to the dependence of density on mixture fraction in a flame with fast chemistry. A fluctuation in mixture fraction will lead to sound generation.

\section{9:15}

5aNSb4. Prediction and reduction of centrifugal blower noises. Christopher L. Banks and Sean F. Wu (Dept. of Mech. Eng., Wayne State Univ., 5050 Anthony Wayne Dr., Detroit, MA 48202)

This paper presents the results of an investigation of centrifugal blower noises. Experiments on three different types of blowers were conducted to determine the major noise generation mechanisms. The information thus gained has led to an innovative noise reduction method utilizing a stationary, noncontacting transition mesh. Experiments showed that an overall noise reduction of 3 to $5 \mathrm{~dB}$ was achieved for all the blowers running at impeller speeds in the range of 10 to $30 \mathrm{~m} / \mathrm{s}$. In addition, a semianalytical model was developed to predict noise spectra from dimensionally similar centrifugal blowers. This model yielded an acoustic power proportional to the fourth power of the blower speeds. Comparisons of the calculated and measured noise spectra from different blowers running under various speeds were demonstrated. Good agreements were obtained in all cases. [Work supported by Ford Motor Company.]

FRIDAY MORNING, 26 JUNE 1998

CASCADE BALLROOM I, SECTION C (W), 9:45 TO 10:45 A.M.

\title{
Session 5aNSc
}

\section{Noise and Architectural Acoustics: Soundscapes-“"Acoustical Landscapes" in Natural and Built Environments}

\author{
Joseph Pope, Chair \\ Pope Engineering Company, P.O. Box 236, Newton Center, Massachusetts 02159
}

\section{Contributed Papers}

\section{9:45}

5aNSc1. Supportive sound environments in building research. Birgitta Berglund (Inst. of Environ. Medicine and Dept. of Psych., Stockholm Univ., Stockholm, Sweden), Edward Hojan, and Anna Furmann (Inst. of Acoust., Adam Mickiewicz Univ., Poznan, Poland)

The aim of this research is to create good sound environments in rooms of ordinary buildings (schools, dwellings, offices, hospitals) for people with hearing deficits. If the deterioration of hearing with age is considered, this group of people may represent a majority rather than a minority of the population. From the many evaluation criteria of natural sounds, both stationary and nonstationary and in room environments, used by subjects, the authors have identified independent ones. These criteria may be named attributes and primarily define the "quality" of the soundperception space in rooms. New experiments involving perceptual evaluation of sound and environments by 40 randomly chosen subjects and the use of multidimensional data analysis confirm in part our earlier results regarding the particular evaluation criteria applied [Furmann et al., J.
Acoust. 4, 535-551 (1991)]. According to Stevens' argument, it seems that the relationship between the values of the evaluation scale and the perceptions are identical for people of normal and impaired hearing although their perceptions may differ.

\section{0:00}

5aNSc2. Features of Japanese soundscapes recognized by foreigners: A questionnaire survey on sounds for the foreigners living in Fukuoka. Shin-ichiro Iwamiya (Dept. of Acoust. Design, Kyushu Inst. of Design, 4-9-1, Shiobaru, Minami-ku, Fukuoka, 815-8540 Japan, iwamiya@kyushu-id.ac.jp)

A questionnaire survey on odd sounds for foreigners living in Fukuoka was done to clarify the feature of Japanese soundscapes recognized by those whose cultural background is different from the Japanese. The foreigners were asked to point out the sound which they usually heard in Japan but heard less in their home countries and their impressions, and the 
sound which they usually heard in their home countries but heard less in Japan and their impressions. The traffic sound signal for the blind, the exhaust noise by wild bike riders, the peddlers' cries, and the election campaign broadcast are the typical sounds only heard in Japan. The foreigners usually have a good image for the traffic sound signal for the blind and the peddlers' cries. In contrast, they have a negative impression on the muffler noise by wild riders and speeches concerning election. The Japanese soundscape is characterized by these sounds which we hear in daily life. Comparison between the sounds usually heard in Japan and those heard less in Japan shows that there are too many public sounds, such as announcements using public-address systems and music at the shopping centers, and few natural sounds and human voices in Japan.

\section{0:15}

5aNSc3. The acoustic and sound design for a museum exhibition on indigenous music and dance. Peter G. Coulter (Museum of Appl. Arts and Sci., P.O. Box K346, Haymarket, Sydney, Australia 2000)

Museum exhibitions commonly use visual design to orientate and inform the visitor. At the Powerhouse museum in Sydney, Australia, an exhibition (entitiled Ngaramang Bayumi) was recently developed on indigenous music and dance. This exhibition was designed to orient the visitor using aural cues, in addition to the visual. This has been achieved by the use of acoustic design and through the use of a specialized sound systems that presents a 3-D sound (ambisonics), that calls and guides the visitor through the space. The experience, along with audiovisuals, objects, text, and graphics uses unusual geometries, layouts, and building materials. Using this design as a case study, this presentation will explain the research and development of the acoustic elements within the exhibition. These are the elements of cross talk, sound quality, sound focusing, sound isolation, sound ambience, localization, dynamic range, and reverberation times. Visitor reaction to the exhibition will also be discused.

\section{0:30}

5aNSc4. Survey on the actual condition of the sound environment in commercial spaces. Michiko So and Sho Kimura (Dept. of Architecture, College of Sci. Technol., Nihon Univ., 1-8 Kanda-Surugadai, Chiyoda-ku, Tokyo, 101 Japan, czf01070@ niftyserve.or.jp)

Many people use a great variety of commercial spaces in their daily life, and are exposed to such a peculiar sound environment both consciously and unconsciously. In order to gain a comprehension of the user's awareness of the sound environment in commercial spaces, a survey was conducted on residents of the Tokyo Metropolitan Area (1198 effective responses were received). The result shows a great majority of those questioned regard the sound environment as one of the features that enhance the atmosphere or the character of commercial spaces. Accordingly, the design of the sound environment plays a role as one of the important factors in creating the atmosphere of individual commercial spaces. On the other hand, it was also revealed that under current conditions, the loudness, articulation, quality, the manner of presentation of background music and announcements, etc. (especially how to use PA systems in noisy surroundings), in individual commercial spaces were not adequately adjusted or designed in many cases. This is associated with the limits of preference and the potential question of the awareness of the users.

FRIDAY MORNING, 26 JUNE 1998

GRAND BALLROOM A (S), 9:15 A.M. TO 12:15 P.M.

\title{
Session 5aPAa
}

\section{Physical Acoustics: Sonochemistry and Sonoluminescence: SL I}

\author{
Thomas J. Matula, Cochair \\ Applied Physics Laboratory, University of Washington, 1013 NE 40th Street, Seattle, Washington 98105 \\ R. Glynn Holt, Cochair \\ Aerospace and Mechanical Engineering, Boston University, 110 Cummington Street, Boston, Massachusetts 02215
}

Chair's Introduction-9:15

Invited Papers

9:20

5aPAa1. Nonlinear bubble dynamics and light emission in single-bubble sonoluminescence. Felipe Gaitan (Natl. Ctr. for Physical Acoust., University, MS 38677) and Glynn Holt (Boston Univ., Boston, MA 02215)

The paradigm system for studying light emission from bubbles has been a single, acoustically levitated air bubble in water. Such a bubble, whose volume pulsations are forced by the time-varying portion of the levitating acoustic field, has a rich dynamic life over a large range of the driving pressure-bubble size parameter space. Measurements have been made of bubble dynamics over a large part of a bubble's parameter space, including the light emission regime. In the context of these measurements, the relationship between observed light emission and the activity of the oscillating bubble will be discussed. In particular, it will be explained how the constraints of mechanical stability and mass flux stability dictate what all experiments in SBSL have seen, namely a very small window of parameter space where light is observed to be emitted. Experimental evidence will be presented to make a clear distinction between features of SL which are due to the mechanism for light emission and those that are due to the dynamics of gas bubbles. [Work supported by NASA and the Office of Naval Research.] 
5aPAa2. Pulse width and shape in single bubble sonoluminescence. Bruno Gompf, Gerald Nick, Rainer Pecha, and Wolfgang Eisenmenger (1.Physikalisches Institut, Universitaet Stuttgart, Pfaffenwaldring 57, D-70550 Stuttgart, Germany)

To test the different theoretical models describing the light emitting process in SBSL measurements of the width and shape of the emitted light pulses are essential. The light pulses have been characterized by two independent methods: with time-correlated single photon counting and with a streak camera. Both methods lead to the same results. The pulse width strongly depends on the parameter's driving pressure, gas and gas concentration and temperature and varies between $60 \mathrm{ps}$ and more than $300 \mathrm{ps}$, and there is no difference between the red and uv part of the spectrum. The streak camera results additionally show that the pulse shape is slightly asymmetric with a steeper ascent and a slower descent. This asymmetry increases with decreasing temperature.

\section{0:00}

5aPAa3. Predictions for upscaling sonoluminescence. Sascha Hilgenfeldt and Detlef Lohse (Dept. of Phys., Univ. of Marburg, Renthof 6, 35032 Marburg, Germany, lohse@stat.physik.uni-marburg.de)

Detailed comparison between the hydrodynamical/chemical approach towards single bubble sonoluminescence (SBSL) and recent experimental data is offered. In particular, the water temperature dependence is focused on. Moreover, detailed predictions of how to upscale SBSL are presented.

10:20

5aPAa4. Single-bubble sonoluminescence and liquid fracture. A. Prosperetti (Dept. of Mech. Eng., Johns Hopkins Univ., Baltimore, MD 21218)

Attention is drawn to the fact that the very mechanism that traps a bubble in a standing acoustic wave causes it to execute an oscillatory translational motion in the direction of gravity. A bubble driven below resonance will move up during the collapse phase and down during the expansion phase. A bubble translating and collapsing develops a jet in the direction of motion. It is hypothesized that sonoluminescence is due to the collision of this jet with the other side of the bubble surface. The mechanism of light emission is a "fracture" process of the liquid that initially cannot respond by flowing due to the very short rise time of the applied pressure. The picosecond duration of the light flash is the time it takes for the microjet overpressure to be relieved by reflection from the microjet free surface. Several other observed features of sonoluminescence (such as noble gas and temperature sensitivity, anomalous mass loss process, effect of surfactants) can also be explained, at least qualitatively. [Work supported by the Office of Naval Research.]

\section{0:40}

5aPAa5. Conditions during multibubble sonoluminescence. Kenneth S. Suslick, William B. McNamara III, and Yuri Didenko (Dept. of Chemistry, Univ. of Illinois, 601 S. Goodwin Ave., Urbana, IL 61801, ksuslick@uiuc.edu)

Acoustic cavitation results in extraordinary transient conditions inside the collapsing bubble. In addition to interesting chemical effects (sonochemistry), cavitation also produces light emission. Such sonoluminescence from cavitating clouds of bubbles ["multibubble sonoluminescence"' (MBSL)] in room-temperature liquids closely resembles flame emission. Effective emission temperatures have been obtained for MBSL from excited state metal atom emission (from sonolysis of several volatile metal carbonyls). From the relative intensities of multiple line emissions from $\mathrm{Cr}$, $\mathrm{Mo}$, and $\mathrm{Fe}$, emission temperatures have been calculated and are all in close agreement with each other. The effects of bubble contents can alter the observed temperatures and this has been now directly observed for the first time. In addition, effective pressures can be estimated from line broadening and line shifts of the metal atom emission. The effective transient conditions formed during cavitation of bubble clouds are roughly $5000 \mathrm{~K}, 500 \mathrm{~atm}$, which implies heating and cooling rates in excess of $1 \mathrm{e} 10 \mathrm{~K} / \mathrm{s}$. Temperatures reached during single bubble sonoluminescence (SBSL) are likely to be very much higher.

\section{1:00-11:10 Break}

\section{1:10}

5aPAa6. Sonoluminescence and its neighbor cavitation bubble luminescence. Werner H. Lauterborn and Thomas Kurz (Drittes Physikalisches Institut, Universität Göttingen, D-37073 Göttingen, Germany)

The field of sonoluminescence, the making of light from sound, and the related field of cavitation bubble luminescence are reviewed. The single-bubble acoustic trap invented by Crum has started great activities through the enhanced possibilities of observing the dynamics of a single bubble in great detail. The question of spherical versus aspherical collapse in light emission and problems of enhancing the light output are addressed. For sonochemistry the case of many bubbles versus a single bubble is important. Some results on the dynamics of multi-bubble systems and their light emission are given. One neighbor of sonoluminescence, i.e., cavitation bubble luminescence from laser-induced bubbles, may be of great help for clarifying some aspects of light emission from collapsing bubbles. 


\title{
Contributed Papers
}

\section{1:30}

5aPAa7. Predictions for stable sonoluminescence in nonaqueous liquids. Michael P. Brenner (Dept. of Math., MIT, Cambridge, MA 02139), Sascha Hilgenfeldt, and Detlef Lohse (Univ. of Marburg, Marburg, Germany)

The oscillation behavior of single sonoluminescing bubbles, and therefore their stability and the intensity of light emission, depends on a multitude of material parameters, including viscosity, surface tension, and liquid vapor. It is shown in analytical and numerical computations how substitution of water by nonaqueous fluids affects the domain for stable and unstable sonoluminescing bubbles in parameter space.

\section{1:45}

5aPAa8. Novel techniques for manipulating single-bubble sonoluminescence. Kirk Hargreaves, Thomas J. Matula, Lawrence A. Crum (Appl. Phys. Lab., Univ. of Washington, Seattle, WA 98105), and William C. Moss (Lawrence Livermore Natl. Lab., Livermore, CA 94550)

Various acoustical methods are currently being utilized to manipulate the radial oscillations of single bubbles undergoing sonoluminescence. Previous measurements have shown that the addition of higher harmonics to the drive frequency [J. Holzfuss, R. Mettin, and W. Lauterborn (private communication)] can result in an increase in the light intensity by as much as $50 \%$. The radial response is also dramatically affected by the addition of higher harmonics. Nonlinear bubble dynamics equations agree well with the measured radial response. This and related work will be described. [Work supported by NSF and under the auspices of the U. S. Department of Energy at Lawrence Livermore National Laboratory under Contract No. W-7405-Eng-48.]

\section{2:00}

5aPAa9. Abrupt drive pressure variations for probing the extinction threshold mechanism for single-bubble sonoluminescence. Thomas $\mathrm{J}$. Matula and Lawrence A. Crum (Appl. Phys. Lab., Univ. of Washington, Seattle, WA 98105)

A novel method utilizing abrupt drive pressure amplitude changes has been used to probe transient conditions in single-bubble sonoluminescence (SBSL). This technique has been used to probe the incipient luminescence threshold [Matula and Crum, Phys. Rev. Lett. (submitted)], where it was found that sonoluminescence from air bubbles depend on the time the bubbles spend in the sonoluminescing state. The abrupt pressure change technique has also recently been used to probe SBSL behavior near the extinction threshold. Simultaneous measurements of the radial response and light emission may be used to better understand the mechanism for SBSL extinction.

FRIDAY MORNING, 26 JUNE 1998

ASPEN ROOM (S), 9:15 TO 10:45 A.M.

\section{Session 5aPAb}

\section{Physical Acoustics: Acousto-Optics and Opto-Acoustics}

\author{
Harry Simpson, Chair \\ Naval Research Laboratory, Code 7136, 4555 Overlook Avenue, SW, Washington, DC 20375-5000
}

\section{Contributed Papers}

9:15

5aPAb1. Planar backward projection of transient fields obtained from optical imaging methods. Gregory Clement, Ruiming Liu, Stephen Letcher (Dept. of Phys., Univ. of Rhode Island, Kingston, RI 02881,gcle0008@uriacc.uri.edu), and Peter Stepanishen (Univ. of Rhode Island, Kingston, RI 02881)

A polarimetric fiber-optic detection system is used to reconstruct the field in front of a planar source. The field is then back-projected toward the source using a filtered wave vector/frequency domain method. This method is compared with a second temporal projection technique that records data over space at a specific time and projects the field to an earlier time. Numerical results will be presented to illustrate the two methods. Experimental results from a pulsed ultrasonic transducer will be presented and discussed.

\section{9:30}

5aPAb2. Measurement of ultrasonic displacement vector with an $F-P$ interferometer. Menglu Qian, Yongdong Pan (Inst. of Acoust., Tongji Univ., Shanghai 200092, PROC), and Zhongxian Zhang (Zhejiang Univ., Hangzhou 310027, PROC)

The laser interferometer technique for receiving ultrasound has potential for a wide range of noncontact ultrasonic measurements and has therefore received considerable interest in recent years. Several interferometric methods for detecting the normal displacement components of ultrasound have been developed and applied to nondestructive evaluation for material characterization. In order to study the propagation of ultrasound in a medium and to characterize materials more accurately, detecting both normal and tangent components of ultrasonic displacement simultaneously is necessary. Therefore, an $F-P$ interferometer, which receives scattering lights at scattering angles $+\theta$ and $-\theta$ from sample surfaces and measures the ultrasonic displacement vector simultaneously, is introduced in this paper. In order to increase the scattering light intensity and determine the scattering angle $\theta$ precisely, a new method for modification of a sample surface is introduced and some experiments are presented in this paper.

\section{$9: 45$}

5aPAb3. Two-frequency degeneracy of an acousto-optic interaction in paratellurite. Alexander Yurchenko and Vladimir Moskalev (Dept. of Quantum Radiophys., Kiev Taras Shevchenko Univ., 64, Vladimirskaya str., 252033 Kiev, Ukraine, alyur@boy.rpd.univ.kiev.ua)

Earlier unknown effects of an acousto-optic interaction degeneracy in paratellurite have been studied. Its essence compared to described phenomena lies in the fact that a light beam can be diffracted twice by acoustic waves with different frequencies instead of being typically diffracted twice by the same acoustic wave. It happens due to strong acoustic anisot- 
ropy of paratellurite. Bragg conditions resulting in the degeneracy can be satisfied under a certain frequency correlation of two acoustic slow shear waves. A light beam diffracted by one spectral component of a complex acoustic signal can be effectively diffracted again by another spectral component. Thus, a physical mechanism of this effect is the same as it is in the ordinary case but it is observed under different conditions (different frequencies). Necessary calculations of such interactions have been carried out. The frequency of the "unfavorable" spectral component was calculated depending on the frequency of the "useful" one for the case of nonaxial acousto-optic interaction in paratellurite. The found effect was shown to take place and to be important for real acousto-optic devices. In the studied case, the frequencies of acoustic waves interacting with a light beam were equal to 44.7 and $43.2 \mathrm{MHz}$, respectively.

\section{0:00}

5aPAb4. Visualization of a phase structure of sound field in a Bragg cell. Vadim Goncharov, Leonid Ilchenko, Vladimir Moskalev, Eugene Smirnov, and Alexander Yurchenko (Dept. of Quantum Radiophys., Kiev Taras Shevchenko Univ., 64, Vladimirskaya str., 252033 Kiev, Ukraine, gva@boy.rpd.univ.kiev.ua)

A phase structure of a sound field in a Bragg cell was investigated experimentally using a dual-beam, dual-frequency laser system. The technique used allows the recording of a spatial phase structure of diffracted light field to visualize phase nonuniformities of a sound field. The underlying physical principle of this technique is to probe an analyzed phase object using two slightly separated in space light beams with different optical frequencies. A difference frequency signal, which is obtained as a photodetector output in an optical heterodyne system, contains information about a phase difference of the adjacent areas of the sound field. When the probing beams are diffracted by a Bragg cell, their optical frequencies are shifted by the same acoustic frequency, so that the difference is the same at any Bragg cell operating frequency. A Bragg cell on paratellurite operating around $100 \mathrm{MHz}$ was investigated. Focused laser beams with a difference of optical frequencies equal to $1 \mathrm{MHz}$ were used as probing beams. Pictures were obtained which demonstrated dependencies of a phase structure of diffracted light on frequency and sound power. Phase nonuniformities across the sound field up to three wavelengths have been estimated. [Work supported by EOARD.]

\section{0:15}

5aPAb5. Radiation acoustics-photoacoustics and acousto-optics of penetrating radiation. Leonid M. Lyamshev (N. N. Andreev Acoust. Inst., Shvernik st. 4, 117036 Moscow, Russia, bvp@asu.acoins.msk.su)

Photoacoustics is a science about optical generation of sound in a substance. The study of light diffraction by acoustic waves is the subject of acousto-optics. Light is a beam of particles, i.e., photons. In this connection the study of sound generation by beams of electrons, protons, neutrons, and other particles in a substance, as well as the study of matter waves' (particle beams) diffraction by ultrasound may be considered correspondingly as photoacoustics and acousto-optics of penetrating radiation. Combined together, this is the field which got the name of radiation acoustics. It develops at the interface of acoustics, nuclear physics, solid state physics, and physics of high energy and elementary particles [see L. M. Lyamshev, Radiation Acoustics (Nauka-Fizmatgiz, Moscow, 1996), in Russian]. Some universal laws characterizing generation of sound by beams of penetrating radiation are discussed. The problem of acousticradiational interaction is considered using the examples of diffraction of $\mathrm{x}$ rays and thermal neutrons by ultrasound in solids. [Work supported by RFBR.]

\section{$10: 30$}

5aPAb6. Photoacoustic signal in the system of a thin oil layer on the water surface. Antoni Sliwinski, Stanislaw Pogorzelski, and Janusz Szurkowski (Inst. of Experimental Phys., Univ. of Gdansk, 80-952 Gdansk, Poland, fizas@univ.gda.pl)

Thin layers of different kinds of oils spread on the water surface (using standard techniques) were examined by photoacoustic spectroscopy (PAS) methods. Both techniques of excitation were applied: the classic one with a modulated light beam as well as with a laser pulse beam. It was demonstrated that the mechanism of molecular interaction at the oil-water interface represents a good model for determination of the correlation between PAS signal characteristics (amplitude and phase) and physicochemical properties of the thin layer system. The results obtained have confirmed the existence of a very sharp separation of the oil and water phases. Variations in physical properties of layers of different thickness like surface and interface tension thermal conductivity and heat capacity and optical absorption coefficients influenced by different agents (e.g., surfactants) could be detected with the PAS methods applied. [Work supported by $\mathrm{KBN}$.] 


\title{
Session 5aPP
}

\section{Psychological and Physiological Acoustics: A Microbrew of Speech Perception and Hearing Impairment (Poster Session)}

\author{
Kathryn H. Arehart, Cochair \\ Department of Speech Language and Hearing Science, University of Boulder, Box 409, Boulder, Colorado 80309 \\ Shari L. Campbell, Cochair \\ University of Georgia, 514 Aderhold Hall, Athens, Georgia 30602
}

\section{Contributed Papers}

\begin{abstract}
All posters will be on display from 7:45 a.m. to 10:45 a.m. To allow contributors an opportunity to see other posters, contributors of odd-numbered papers will be at their posters from 7:45 a.m. to 9:15 a.m. and contributors of even-numbered papers will be at their posters from 9:15 a.m. to 10:45 a.m. To allow for extended viewing time, authors have been requested to place their posters on display on Thursday afternoon beginning at 1:00 p.m. in Grand Ballroom II.
\end{abstract}

5aPP1. Perceptual consequences of amplitude perturbation in the wavelet coding of speech. Nicolle H. van Schijndel, Tammo Houtgast, and Joost M. Festen (Dept. of Otolaryngol., Univ. Hospital VU, P.O. Box 7057, 1007 MB Amsterdam, The Netherlands, NH.vanSchijndel@azvu.nl)

Wavelet coding of sound is a frequency-time representation that may be able to mirror the properties of auditory coding at a peripheral level, provided that we use the proper so-called mother wavelet. Previous experiments based on intensity discrimination [van Schijndel et al., J. Acoust. Soc. Am. (submitted)] suggested a Gaussian-shaped wavelet with a bandwidth of $1 / 3$ oct. Based on this function, a wavelet coding and reconstruction algorithm has been developed. The frequency-time plane is split up: one wavelet per three periods along the time axis and nine wavelets per octave along the frequency axis. In this study the amplitude of the wavelet coefficients is manipulated before the stage of reconstruction. The perceptual effect of amplitude perturbation on speech perception is studied. A (partial) answer to the following questions will be presented: (1) what degree of amplitude perturbation is just noticeable to the subject, and (2) how is speech intelligibility affected by suprathreshold amplitude perturbation. The results may provide insight into the acuity of the auditory amplitude coding and its effect on speech intelligibility. [Work supported by the Netherlands Organization for the Advancement of Pure Research (N.W.O.).]

5aPP2. The effect of spectral resolution on speech perception in a multitalker babble background. Bom Jun Kwon and Christopher W. Turner (Dept. of Speech Pathol. and Audiol., Univ. of Iowa, Iowa City, IA 52242)

Does finer spectral resolution always have an advantage for speech recognition? In this experiment, speech was divided into separate bands within which spectral information was removed by replacing the fine structure with noise; the temporal envelope cues of each band remained intact. Normal-hearing subjects identified consonants in $\mathrm{a} / \mathrm{C} / \mathrm{a}$ for speech and babble processed with 1, 2, 4, and 8 bands, in addition to the unprocessed speech and babble, for a range of signal-to-babble ratios. For high S/B ratios our results agree with earlier results, in that performance improved with increases in the number of bands, and higher transmission of information was observed for voicing and manner than place of articulation [Shannon et al., Science 270, 303-304 (1995)]. However, as S/B decreased, the advantage of better spectral resolution was reduced. This suggests that for speech in a competing babble background, increased spectral resolution not only increases the saliency of speech, but at the same time makes background babble a more effective distracter. Thus the benefit provided by fine spectral resolution depends upon the task and S/B ratio. [Work supported by NIDCD.]

5aPP3. Application of auditory models to discrimination thresholds of voicing parameters. Shari L. Campbell (Dept. of Commun. Sci. and Disord., Univ. of Georgia, 514 Aderhold Hall, Athens, GA 30602), Kathryn H. Arehart (Univ. of Colorado, CB 409, Boulder, CO 80309), Ronald C. Scherer (Bowling Green State Univ., Bowling Green, $\mathrm{OH}$ 43403), and Amy N. Butler (Univ. of Georgia, Athens, GA 30602)

A recent study [Scherer et al., J. Voice (to be published)] reported discrimination thresholds for synthesized complex tones emulating glottal flow waveforms and output pressure waveforms for the vowel /a/. Each signal type was varied along both an open quotient and a speed quotient continuum using standard values associated with normal voice production. jnds for the output pressure signals were significantly larger than for the glottal flow signals. The current experiment compared quantities derived from "auditory-perceptual" representations of the acoustic signals. These quantities (centers of gravity and Euclidean distances) were computed for both the raw amplitude spectra and for modeled excitation patterns and specific loudness patterns. Overall loudness and overall sharpness were also estimated. The comparisons indicated ways in which auditory modeling can enhance our understanding of basic issues in voice and speech perception. [Work supported by UGA College of Education.]

5aPP4. Audio signals in domestic appliances evaluated in terms of the hearing ability of older adults. Kenji Kurakata, Yasuyoshi Kuba, Yasuo Kuchinomachi (Natl. Inst. of Bioscience and Human-Technol., 1-1 Higashi, Tsukuba, Ibaraki, 305 Japan, kurakata@nibh.go.jp), and Kazuma Matsushita (Natl. Inst. of Technol. and Evaluation, Tokyo, 151 Japan)

The audio signals used in domestic appliances currently available on the market in Japan were recorded to identify suitable signals for the hearing ability of older adults. The results of the analysis indicated the following three problems: (1) Some appliances use high-frequency tones around $4000 \mathrm{~Hz}$. Since these sounds are hard for older adults with presbycusis to hear, it would be better to use signals with lower frequencies. However, the problem here is that if the frequency is lowered, then the signal might be masked by domestic sounds whose power would be greater relative to the low-frequency signals. (2) The signals used by some 
appliances are too soft. The intensity of some signals should be adjusted to compensate for the hearing loss among older adults. (3) The sounds used in these appliances are often very similar in terms of both timbre and temporal ringing patterns. That may cause confusion because it is difficult to identify which appliance is signaling.

5aPP5. The effects of age and hearing impairment on the time course of backward masking. Sara Elizabeth Gehr and Mitchell Sommers (Washington Univ., Dept. of Psych., 1 Brookings Dr., Campus Box 1125, St. Louis, MO 63130)

The present study was designed to assess the effects of age and hearing loss on the time course of backward masking. Thresholds for detecting a $10-\mathrm{ms}, 500-\mathrm{Hz}$ tone were measured as a function of the temporal separation between the signal and a 50-ms broadband masker in normal-hearing young listeners (18-25), normal-hearing older adults (65-82), and hearing-impaired older adults. Younger subjects exhibited less initial masking, had steeper recovery functions, and asymptoted closer to their unmasked thresholds than either group of older adults. Comparisons between the normal-hearing and hearing-impaired older individuals indicated a slower time course of recovery from backward masking for listeners with hearing loss. Differences between the two groups of normalhearing listeners (young and old) suggest that age, independent of hearing loss, affects the temporal course of backward masking. Differences between the normal-hearing and hearing-impaired elderly listeners, however, indicate that age-related hearing impairment may also alter the rate of recovery from backward masking. [Work supported by the Brookdale Foundation and NIA.]

5aPP6. Speech intelligibility and localization in a complex environment by listeners with hearing impairments. Monica L. Hawley, Ruth Y. Litovsky, and H. Steven Colburn (Hearing Res. Ctr. and Dept. of Biomed. Eng., Boston Univ., Boston, MA 02215)

Listeners with hearing impairments often complain of difficulties when trying to communicate in a complex environment such as a cocktail party. Explorations of the dependence of the intelligibility and localizability of speech on the number and spatial positions of simultaneous interfering sources are reported on. Target sources are sentences spoken by a female and interfering sources include other sentences or speech-shaped noise. The total number of sources played at any one time varied between one and four. Listeners with normal hearing, profound monaural deafness, and with symmetric sensorineural hearing impairments were tested. Listeners were tested without hearing aids. Previous work in free field and with virtual stimuli has shown that bilaterally hearing-impaired listeners show large intersubject differences in their performance in both speech intelligibility and localization tasks. In the present study an investigation was made to see whether these intersubject differences are related to each listener's sensitivity to specific binaural cues. Therefore, in addition to localization and speech intelligibility, performance in discrimination of interaural time and level, as well as binaural masking level differences in each listener was measured. The goal of this study is to determine the extent to which performance on speech-based tasks can be accounted for by basic binaural abilities. [Work supported by NIH-NIDCD DC00100.]

5aPP7. Monotic and dichotic musical-interval identification in listeners with cochlear-based hearing loss. Kathryn H. Arehart (Univ. of Colorado, Speech, Lang., Hearing Sci., CB 409, Boulder, CO 80309) and Edward M. Burns (Univ. of Washington, Seattle, WA 98195)

This study examined musical-interval identification in musically experienced listeners with cochlear-based hearing loss. Sounds had missing fundamentals ranging from $100-500 \mathrm{~Hz}$. These missing fundamentals were conveyed by two successive harmonics presented either to the same ear or to separate ears. Performance was measured as a function of the average rank of the lowest harmonic for both monotic and dichotic con- ditions at both $20 \mathrm{~dB}$ sensation level (SL) and $30 \mathrm{~dB}$ SL. In both monotic and dichotic conditions, listeners with cochlear-based hearing loss demonstrated excellent musical-interval identification at low fundamental frequencies and low average ranks, but abnormally poor recognition at higher fundamental frequencies and higher average ranks. The results will be related to auditory deficits which might affect complex-tone pitch perception in listeners with cochlear-based hearing loss, and will be discussed in the context of modern pitch theories. [Work supported by Deafness Research Foundation.]

5aPP8. Minimum bandwidth required for speech-reception by normal-hearing and hearing-impaired listeners. Ingrid $M$. Noordhoek, Tammo Houtgast, and Joost M. Festen (Dept. of Otolaryngol., Univ. Hospital VU, P.O. Box 7057, 1007 MB Amsterdam, The Netherlands, im.noordhoek@azvu.nl)

A new adaptive test has been developed to determine the bandwidth of speech (with a center frequency of $1 \mathrm{kHz}$ ) required for $50 \%$ intelligibility. Measuring this speech-reception bandwidth threshold (SRBT), in addition to the more common speech-reception threshold (SRT) in noise, may be useful in investigating the factors underlying impaired suprathreshold speech perception. A wider-than-normal SRBT points to a deterioration in suprathreshold sound processing in the $1-\mathrm{kHz}$ frequency region. Both the SRT in noise and the SRBT were measured for 10 normal-hearing and 34 hearing-impaired listeners. To keep the speech signal above hearing threshold for all frequencies, speech and noise spectra were shaped to fit in the dynamic range of individual listeners. The average SRBT of the normal-hearing listeners was 1.4 oct. Most hearing-impaired listeners needed a wider bandwidth to reach 50\% intelligibility. Some wider SRBTs were predicted by the SII model (speech intelligibility index) from a high presentation level or narrow dynamic range. The remaining differences between the performance of normal-hearing and hearing-impaired listeners are probably caused by an impaired suprathreshold sound processing in the $1-\mathrm{kHz}$ frequency region. [Work supported by the Foundation "Heinsius-Houbolt Fonds.']

5aPP9. Loudness of dynamic stimuli in cochlear-impaired listeners. Chaoying Zhang and Fan-Gang Zeng (House Ear Inst., 2100 West Third St., Los Angeles, CA 90057)

In normal hearing listeners, a temporally fluctuating sound with modulating frequencies from 4 to $400 \mathrm{~Hz}$ was found to be louder than predicted by its averaged power. The louder sensation due to temporal fluctuation was also found to be overall level-dependent: approaching peak amplitude loudness at a comfortably loud level, becoming less at lower sensation levels, and disappearing at higher levels. If this level-dependent loudness effect were related to the nonlinear cochlear compression, then it would disappear in cochlear-impaired listeners because their nonlinear compression is presumably damaged. Three listeners with clinically diagnosed cochlear impairment balanced loudness between white noise and sinusoidally amplitude-modulated noise. The results showed that the modulated noise was louder for modulating frequencies between 4 and $400 \mathrm{~Hz}$ at all three sensation levels, consistent with the hypothesis that the overall level effect on loudness of dynamic stimuli is related to the cochlear compression. These results indicate that loudness of dynamic stimuli is governed by two different mechanisms: a retrocochlear temporal processing mechanism which is presumably preserved in both normal and cochlear-impaired listeners, and a cochlear mechanism which provides amplitude compression at high levels in normal listeners and is damaged in the cochlearimpaired listeners. [Work supported by NIDCD.] 
5aPP10. Further effects of cochlear loss on ICP loudness adaptation, and loudness constancy. Rebecca Schnieder Ludwig, Hongwei Dou, Ernest M. Weiler, Laura W. Kretschmer, David E. Sandman (ML \#379, CSD, Univ. of Cincinnati, Cincinnati, OH 45221), and Eleanor Stromberg (Cincinnati Speech \& Hearing Ctr.)

ICP loudness adaptation depends on an ipsilateral comparison paradigm with a short increasing or decreasing referent value to reveal loudness adaptation. Janson et al. [Br. J. Audiol. 29, 288-297 (1996)] found the intensity-based function for ICP loudness adaptation for those with a high-frequency loss differed significantly from normal listeners. Loss of loudness constancy in normal listeners was consistent, loss in those with a cochlear loss showed a negative progression as intensity increased, as if it were the inverse of recruitment. The purpose of the present study was to compare ICP adaptation to recruitment. ICP adaptation and recruitment were tested in ten high-frequency loss (probably noise induced) and ten age-matched normal hearing individuals. Although the evidence for recruitment was generally found in those with hearing loss, there was no simple relationship to ICP adaptation. Variation among measures of recruitment, implications of abnormal loudness constancy for speech perception, and the possible use of measures of otoacoustic emissions for future studies will be discussed.

5aPP11. Evoked otoacoustic emissions, suppression and comparison to loudness constancy, at speech frequencies. Ernest $M$. Weiler, Hongwei Dou, Robert S. Tannen, David E. Sandman, William N. Dember, and Joel S. Warm (ML \#379, Hearing Lab., CSD, Univ. of Cincinnati, Cincinnati, OH 45221)

Spontaneous otoacoustic emissions (SOAE) reflect spontaneous activity of the outer hair cells of the cochlea. Suppression of transient evoked otoacoustic emissions (TEOAE) bears operational similarity to loudness adaptation determined by the ipsilateral comparison paradigm (ICP). The present study gathered these measures from 41 normal hearing adults (5 male, 36 female, 20-45 years old). There was no significant relationship between spontaneous SOAE, but there was a significant relationship $(r$ $=-0.36, p<0.05)$ between ICP loudness adaptation and suppression of TEOAEs. The relationship between these two active measures has implications for failure of adaptation or the apparent loudness constancy in the speech range revealed by simple adaptation measures. It has been previously noted that the ICP technique yields adaptation in the speech frequencies between 40 and $80 \mathrm{~dB}$, where the simple adaptation apparently does not [Weiler, Sandman, and Dou, program abstract 4pPPa10, J. Acoust. Soc. Am. 101, 3177 (1997)]. Implications will be discussed in terms of the results uncovered in this study.

5aPP12. Virtual midplane localization of subjects with sensorineural hearing loss. Helen J. Simon (Smith-Kettlewell Eye Res. Inst., 2232 Webster St., San Francisco, CA 94115, helen@skivs.ski.org) and Inna Aleksandrovsky (Univ. of California, Berkeley, CA)

This paper reports the results of a study that investigated localization performance of simulated externalized signals in sensorineural hearing loss (SNHL) listeners when signals were presented with either unbalanced [equal sensation level (EqSL)] or balanced [equal sound-pressure level (EqSPL)] hearing aid gain at the two ears. In previous reports from this laboratory, it has been found that when equalizing by SL, the perceived lateral position was essentially linearly dependent on the degree and direction of asymmetry in normal-hearing $(\mathrm{NH})$ and SNHL listeners. Equalizing by SPL showed no such dependency. It was hypothesized that the current practice of fitting "binaural" hearing aids in asymmetric SNHL listeners using prescription formulas might result in SPL imbalance which would disrupt the previously adapted system and impair localization. The present study was designed to address this issue by measuring localization using a simulated externalized auditory image combined with head position control of IID and ITD to spatially fix the image. Absolute and signed error deviations from center were analyzed. Significant interactions of group $(\mathrm{NH}$ versus SNHL)* frequency and balance (EqSL or Eq SPL)* frequency were found. These data will be discussed in terms of auditory adaptation and strategies of fitting binaural hearing aids. [Work supported by NIDRR.]

5aPP13. Use of temporal information in recognition of amplitudecompressed speech by older adults. Pamela E. Souza and Johanna J. Larsen (Dept. of Speech and Hearing Sci., Univ. of Washington, 1417 NE 42nd St., Seattle, WA 98105, psouza@u.washington.edu)

Amplitude compression alters the speech envelope and under some conditions can reduce the availability of temporal cues for speech recognition. This may be a particular problem for older listeners, who demonstrated temporal resolution deficits in some studies. Older and younger hearing-impaired listeners were tested on their recognition of VCV syllables processed using the signal-correlated noise (SCN) method [Schroeder, J. Acoust. Soc. Am. 44, 1735-1736 (1968)]. This method results in a time-varying speech envelope modulating a noise carrier, and preserves temporal cues while minimizing spectral information. Recognition scores were obtained for uncompressed SCN syllables and for the same speech tokens after digital processing with a syllabic compression algorithm. In control conditions, recognition was tested for VCVs that included both temporal and spectral information. Younger and older listener groups were matched for mean hearing thresholds up to $4 \mathrm{kHz}$. A high-pass masker was included to eliminate contributions from the basal end of the cochlea. When compared to younger listeners with similar hearing losses, the older listeners demonstrated poorer performance on the compressed SCN signals. These results suggest that the poorer temporal resolution abilities of older listeners may play a role in their understanding of amplitude-compressed speech.

5aPP14. Apparent effects of the use of digital hearing aid "CLAIDHA" on the several hearing functions of the impaired listeners. Hiroshi Hidaka, Naoko Sasaki, Tetsuaki Kawase, Tomonori Takasaka (Dept. of Otolaryngol., Tohoku Univ. School of Medicine), Kenji Ozawa, Yôiti Suzuki, and Toshio Sone (Res. Inst. of Elec. Commun., Tohoku Univ.)

The portable digital hearing aid system CLAIDHA (compensate loudness by analyzing input signal digital hearing aid), which employs frequency-dependent amplitude compression based on narrow-band compensation, has been developed [Asano et al. (1991)]. The patients with hearing loss using hearing aids experience a new sound world as amplified by the device. It seems to be important to know the apparent change of hearing function by the use of a hearing aid. In the present study, the apparent effects of CLAIDHA amplification especially on the intensity discriminability and the masking function were examined in the patients with hearing loss. The results obtained indicate that the CLAIDHA amplification often improves not only the apparent thresholds but also the apparent masking relation between two tones as compared to simple linear amplification; i.e., in the CLAIDHA amplification, more signals could be heard under the same masking condition. On the other hand, from the viewpoint of intensity discriminability, when the compression ratios of the amplification were larger than two, the IDL (intensity difference limen) rapidly increased more than expected. The advantage of the compressive amplification of the CLAIDHA system will be discussed.

5aPP15. The effect of frequency-modulation systems on the communication skills of deaf-blind children. Barbara Franklin (Dept. of Special Education, San Francisco State Univ., 1600 Holloway Ave., San Francisco, CA 94132, dick3580@aol.com)

Classrooms and other public places provide a poor acoustical environment for individuals who have a hearing loss, and it is often difficult for them to discriminate between the speaker and background noise with a hearing aid. The use of a frequency-modulation (FM) system can often reduce this problem by providing a constant sound pressure level of the 
speaker's voice. The effect of FM systems on communication skills of deaf-blind children was investigated using a single-subject alternating treatment design. There was a marked improvement for a number of the subjects in the FM condition. The results will be discussed along with individual case studies. FM technology has exploded. A relatively new type of FM receiver is now available which combines the hearing aid and FM in a single behind-the-ear unit (BTE/FM). The most recent development is a "boot" attachment which changes a standard over-the-ear hearing aid into a FM system. An overview of these FM systems will be presented. [Work supported by OSERS.]

5aPP16. A metric for determining the degree of compression of a processed signal. Jon C. Schmidt ${ }^{\text {a) }}$ and Janet C. Rutledge $^{b}$ (Dept. of Elec. Eng. and Comput. Sci., Northwestern Univ., Evanston, IL 60208-3118, jschmidt@ resound.com)

When attempting to compare different compression algorithms or different parameter settings of a given compression algorithm, there is a need to compare equivalent amounts of compression. Listening tests that have varied only one parameter while fixing the others have obtained the expected results that increasing the amount of compression that occurs creates a more audible difference between the compressed version and the original. Since there are several parameters that influence the actual amount of compression that occurs, it is desirable to know which set of parameters will create to the least perceptual difference from the original signal for a given amount of compression. Unfortunately, there is not a well-defined approach for determining the amount of compression that has been imparted to a signal. The peak/RMS ratio is woefully inadequate for many compression contexts. Two degree of compression metrics are proposed that can be used to determine the amount of compression by direct analysis of the audio signal before and after compression. These metrics give excellent results in predicting the ratings of subject-based testing on the audio quality of several different audio segments across several parameter variations. ${ }^{\text {a) }}$ Currently with ReSound. ${ }^{\text {b) }}$ Currently with the University of Maryland Medical Center.

5aPP17. Modeling tactile speech perception with auditory simulations. Albert T. Lash, Janet M. Weisenberger, and Ying Xu (Speech and Hearing Sci., Ohio State Univ., Columbus, OH 43210)

A useful technique for gaining insights into the perception of speech stimuli presented via vibrotactile aids is to model this perception by presenting degraded auditory stimuli to normal-hearing observers. It is likely that spectral, temporal, and intensive properties of the tactile system could all affect tactile speech perception. In the present experiments, tactile perceptual data were collected for a set of nonsense syllables presented via a vibrotactile vocoder to normal-hearing subjects. The same subjects were tested with sets of degraded auditory stimuli that were modified by reducing spectral, temporal, or intensive parameters. Spectral reductions alone, achieved by reducing the number of filter channels and broadening filter bandwidths, did not appreciably affect auditory performance, nor did intensity reductions, achieved by reducing dynamic range and intensity quantization. Although temporal reductions, achieved by low-pass filtering the stimuli, did result in lower perceptual scores, the pattern of stimulus confusions did not correlate highly with the tactile data. Pairwise and three-way combinations of spectral, temporal, and intensive parameters yielded more promising correlations. Implications for constructing a comprehensive model of vibrotactile speech perception are discussed. [Work supported by NIH.]
5aPP18. A design of a tactile voice coder with different tactile sensations for the hearing impaired. Chikamune Wada, Shuichi Ino, Hisakazu Shoji, and Tohru Ifukube (Res. Inst. for Electron. Sci., Hokkaido Univ., N12 W6, Kita-ku, Sapporo, 060 Japan, chik@sense.hokudai.ac.jp)

A tactile voice coder is a device which changes a speech sound into a vibratory pattern and presents it to the tactile sense of the hearing impaired. The authors have been improving the tactile voice coder in order that the hearing impaired can identify consonants easier. The use of a convex stimuli pattern, similar to Braille, in addition to the vibratory stimuli is proposed, as one of the ways of improving the tactile voice coder. The authors have made this proposal based on the hypotheses that the combination of the vibratory stimuli and the convex stimuli could transmit more speech information. From the combination of basic experiments, the authors have found that presenting a vowel using the convex stimuli and a consonant using the vibratory stimuli was better than only using the vibratory stimuli. Therefore, based on our findings, the authors have designed a new tactile voice coder which uses a different tactile sensation.

5aPP19. Sensitivity of transiently evoked otoacoustic emission to a long-term exposure of impulsive noise. Amnon Duvdevany and Miriam Furst (Dept. of Eng., Tel-Aviv Univ., Tel-Aviv, Israel, mira@eng.tau.ac.il)

In spite of the regular use of ear protectors, $30 \%-40 \%$ of the soldiers that are often exposed to impulsive blast noise are suffering from hearing loss. The purpose of the present study is to find in what ears click-evoked otoacoustic emissions (COAEs) were influenced by the noise exposure. The preliminary study included testing of 15 healthy soldiers. They were exposed to blast noise (M-16 riffles) accompanied by replicas from a semi-enclosed firing range in two consecutive sessions, followed by half a year of exposure to continuous blast noise (service period). In each stage COAEs were measured. The blast noise was precisely measured with microphones located next to the shooters' ears. None of the soldiers acquired a hearing loss. In most subjects COAE levels have significantly changed in the frequency range $2.5-4 \mathrm{kHz}$. It decreased in the postexposure condition relative to the preexposure by $\sim 3 \mathrm{~dB}$. It increased before the second training session by $\sim 8 \mathrm{~dB}$, and it decreased in the last measurement by $\sim 5 \mathrm{~dB}$. A protection and a destruction mechanism are possibly operating. The protection mechanism reduces the cochlear amplification and causes a reduction in COAE level. The destruction mechanism increases the cochlear nonuniformity which causes an increase in COAE level.

5aPP20. The effects of dexamethasone following an acute acoustic trauma. William A. Ahroon, Ann R. Johnson, B. Sheldon Hagar, and Roger P. Hamernik (Plattsburgh State Univ., Plattsburgh, NY 12901)

An acute acoustic trauma (AAT) results when the unprotected ear is exposed to very high sound pressure levels, usually as the result of an industrial or military accident. There is no commonly accepted treatment in the U.S. for an AAT. A study was performed to evaluate the effects of a corticoid steroid (dexamethasone) on the hearing loss from a series of blast waves that would simulate an AAT. Dexamethasone is commonly used as an anti-inflammatory agent in veterinary medicine. Groups of chinchillas were exposed individually to ten $160-\mathrm{dB}$ peak SPL reverberant blast waves from a conventional shock tube at a rate of one blast per minute. Immediately following the exposure, the animals were injected with dexamethasone alone $(1.0-2.0 \mathrm{mg} / \mathrm{kg}$ IV) or in combination with dimethyl sulfoxide (DMSO), a free-radical scavenger. Individual groups of animals showed the well-known extreme variability in hearing losses as noted in earlier studies [e.g., R. P. Hamernik et al., J. Acoust. Soc. Am. 90, 197-204 (1991)]. Examining the median permanent threshold shifts (PTS) of the groups of animals showed a distinct dose-response effect with increasing dosages of dexamethasone associated with lower levels of PTS in the frequency region most affected by the noise exposure. 
5aPP21. Protective effects of magnesium on noise-induced hearing loss: Animal studies. Fred Scheibe and Heidemarie Haupt (Dept. of Otorhinolaryngology, Charité-Hospital, Humboldt Univ., Schumannstr. 20-21, D-10117 Berlin, Germany, fscheibe@rz.charite.hu-berlin.de)

Magnesium (Mg) deficiency was found to increase noise-induced hearing loss in laboratory animals. This paper reports both prophylactic and therapeutic effects of $\mathrm{Mg}$ on the acoustic trauma produced by a high-level impulse noise series ( $\left.L_{\text {peak }} 167 \mathrm{~dB}, 38 \mathrm{~min}, 1 / \mathrm{s}\right)$. Hearing loss was tested by auditory brainstem response audiometry at frequencies between 0.5 and $32 \mathrm{kHz}$. Permanent hearing threshold shifts (PTS) were measured 1 week after the exposure. For the prophylaxis experiments, anesthetized guinea pigs with either a physiologically high or low Mg status, produced by different diets, were used. For the therapy experiments, animals with the low $\mathrm{Mg}$ status received (immediately after exposure) either Mg injections combined with a Mg-rich diet or saline as a placebo. Total Mg concentrations of perilymph, cerebrospinal fluid, and plasma were analyzed to test the Mg status of the animals. The PTS was found to be significantly lower in the high-Mg group than in the low-Mg group. This also applies to the PTS found between the therapy and the placebo groups. There was a good correlation between the PTS and the perilymphatic Mg. The intracochlear $\mathrm{Mg}$ level seems to play an important role in the acoustic trauma. [Supported by German Defence Ministry.]
5aPP22. Effects of impulse noise on the hearing of fetal sheep in utero. Kenneth J. Gerhardt (Dept. of Commun. Processes and Disord., Univ. of Florida, Box 117420 Gainesville, FL 32611-7420, gerhardt@cpd.ufl.edu), Xinyan Huang, and Robert M. Abrams (Univ. of Florida, Gainesville, FL 32610)

Knowledge of the transmission of exogenous sounds to the fetal head and the effects that these sounds have on fetal hearing is incomplete. The purposes of this study were to measure the transmission of impulse noise into the uterus and to evaluate the effects of impulse noise delivered to pregnant sheep on the hearing of the fetus in utero. A shock tube produced impulses that averaged $169.7 \mathrm{~dB}$ peak sound pressure level (pSPL) in air. In the uterus, the pSPL varied as a function of fetal head location. When the fetal head was against the abdominal wall, peak levels were within 2 $\mathrm{dB}$ of airborne levels and the stimulus resembled a Friedlander wave. When the fetal head was deep within the uterus, the duration of the impulse increased and the peak amplitude decreased. In some instances the decrease in pSPL exceeded $10 \mathrm{~dB}$. Slight elevations of evoked potential thresholds were noted but only for low-frequency stimuli. The integrity of hair cells from these animals was assessed using scanning electron microscopy.

FRIDAY MORNING, 26 JUNE 1998

WEST BALLROOM B (S), 9:15 A.M. TO 12:15 P.M.

\title{
Session 5aSA
}

\section{Structural Acoustics and Vibration: Vibrations of Structural Elements}

\author{
Linda P. Franzoni, Chair \\ Department of Mechanical and Aerospace Engineering, North Carolina State University, Box 7910, \\ Raleigh, North Carolina 27695-7910
}

\section{Contributed Papers}

9:15

5aSA1. Moment and force mobilities of semi-infinite tapered beams. Eugene J. M. Nijman (CRF, Strada Torino 50, 10043 Orbassano, Italy, e.nijman@crf.it) and Bjorn A. T. Petersson (Loughborough Univ., Leicestershire LE11 3TU, UK)

The implications of spatially varying structural properties for flexural vibrations will be addressed for beamlike systems. Under the assumption of pure bending, closed-form expressions for the mobilities of a semiinfinite wedge have been derived. The influence of tapering on the energy flow is analyzed for the structural system constituted by a finite length taper attached to a semi-infinite, uniform beam. It is found that the main distinction from the uniform case is a comparatively broadbanded transition from flexural vibrations governed by the properties of the deep part of the system to vibrations governed by those of the slender part. This transition region is featured by a stiffness-controlled global behavior in the case of a moment-to-rotational response, whereas a global resonant behavior is found in the case of force-to-translational response. It is also demonstrated that constructive and destructive interferences due to reflections from the discontinuity between the two structural members are superimposed on the global behavior. Based on the complete expressions for the configurations studied, estimation procedures for the mobilities will be proposed. In addition, experimental results will be presented which corroborate the description developed and the parameter influence established.
$9: 35$

5aSA2. Vibrational power transmission in asymmetric framework structures. Jane L. Horner (Dept. AAETS, Loughborough Univ., Loughborough, Leicestershire LE11 3TU, England)

When attempting to control the vibration transmitted from, say, a machine into and through the structure upon which it is mounted, it is desirable to be able to identify and quantify the vibration paths in the structure. Knowledge of transmission path characteristics enables procedures to be carried out, for example, to reduce vibration levels at points remote from the source, perhaps with the objective of reducing unwanted radiation of sound. One method for obtaining transmission path information is to use the concept of vibrational power transmission. Many machines are installed on frameworks constructed from beamlike members. By using the concept of vibrational power it is possible to compare the contributions from each wave type. Wave motion techniques are used to determine the expressions for vibrational power for each of the various wave types present. The results from the analysis show the amount of vibrational power carried by each wave type and the direction of propagation. Consideration is given to the effect on the vibrational power transmission of introducing misalignment of junctions in previously symmetric framework structures. Unlike other techniques using vibrational power to analyze frameworks, the model keeps the contributions from each of the various wave types separate 
5aSA3. Vibration transmission via a nonideal beam junction: FEM and analytical combined methods. Igor Grouchetski (Krylov Shipbuilding Res. Inst., 196158 St. Petersburg, Russia) and Eric Rébillard (Chalmers Univ. of Technol., 41296 Gotheburg, Sweden)

The study is devoted to the calculation of the vibration transmission via a nonideal junction; the example considered is an L-beam system including two steel beams and an elastic block between them. A combination of two calculation methods is used. A finite element method (package ABAQUS) is used to determine the stiffness characteristics of the elastic block having a rectangular parallelpiped shape. These stiffness characteristics are substituted into the calculation for the vibrational behavior of the L-beam system. For such a calculation, the transfer matrix method (TMM) is applied. The experimental approach of the study is divided into two different parts. First the tension-compression and the shear stiffnesses of the elastic block are measured, and, second, the transfer mobility through the complete L-beam system with the rubber elastic block between the two beams is measured. For the two cases, calculated and measured results are compared.

\section{0:15}

5aSA4. Measurement of flexural intensity using a dual-mode fiberoptic sensor. Bernard J. Sklanka (Aero/Noise/Propulsion Lab., Boeing Commercial Airplane Group, P.O. Box 3707, Seattle, WA 98124-2207, bernard.j.sklanka@boeing.com), Karl M. Reichard, and Timothy E. McDevitt (Penn State Univ., State College, PA 16804)

An experiment was performed which demonstrated the use of distributed optical fiber sensors to measure power flow in a damped cantilevered beam. Dual-mode optical fiber sensors are an implementation of an optical fiber interferometer in which one fiber acts as both the sensing and reference arms. When bonded to the surface of a structure the sensor provides a distributed measurement of the strain along the length of the sensor. The sensors output is proportional to the integral of the axial component of the strain along the length of the fiber. Two optical fiber sensors were used to measure power flow in a damped cantilevered beam. Finite difference and finite sum approximations were used to determine bending moment and rotational velocity from the output of the fiber sensors. These quantities were then used to calculate the far-field structural intensity. The structural intensity measured using the optical fiber sensors was compared to the structural intensity measured using a two-accelerometer method.

\section{0:35}

5aSA5. The condition for beam-bending modes to dominate in the vibro-acoustic behavior of a circular cylindrical shell. Chong Wang and Joseph C. S. Lai (Acoust. and Vib. Unit, Univ. College, Univ. of New South Wales, Australian Defence Force Acad., Canberra, ACT 2600, Australia, c-wang@adfa.oz.au)

The acoustic radiation from circular cylindrical shells is of fundamental and applied interest primarily because cylindrical shells are widely used in industries. However, according to previous studies, only a few special cases, for example, a cylindrical shell under the assumption of beam bending, can be solved analytically. Obviously, in practice, the vibration behavior of a cylindrical shell may not be assumed to be beam bending in the whole frequency range of interest. This is because the vibration behavior of a cylindrical shell changes with frequency. Therefore, it is important to determine the condition under which a circular cylindrical shell would behave like beam bending so that the analytical solution could be used correctly. In this paper, an analysis of the basic vibration behavior of circular cylindrical shells using the Love's equations shows that for an infinite length cylindrical shell, the beam-bending wave would always be propagating with other flexural waves of different circumferential mode numbers. However, for a finite-length circular cylindrical shell, below the cutoff frequency of $n=2$ mode, beam-bending modes would dominate the vibration response so that it can be treated as a beam with reasonable accuracy. This analytical result is supported by calculations obtained using the boundary element method.
5aSA6. The effect of rib resonances on the vibration and wave scattering of a ribbed cylindrical shell. Martin H. Marcus (Naval Res. Lab., 4555 Overlook Ave., S.W., Washington, DC 20375, marcus@astro.nrl.navy.mil)

Wave phenomena have previously been observed for cylindrical shells with periodically spaced ribs. The understanding of these waves, such as Bragg and Bloch waves, requires knowledge of the periodicity of the ribs and how they interact with each other. In this paper, the understanding of the individual rib resonances gives explanation to the resonance bands that are seen in both experiment and numerical simulations. For these resonances, periodicity becomes less important in computing the frequencies of resonances on the shell and in the far field. However, lack of periodicity in rib spacing and size affects the width and strength of these resonance bands. [Work supported by ONR.]

\section{1:15}

5aSA7. A simple model for structure-borne sound transmission based on direct wave and first reflections. Jian Liang and Bjorn A. T. Petersson (Dept. AAETS, Loughborough Univ., Loughborough, Leicestershire LE11 3TU, England)

In finite structures, the total wave field is composed of the direct wave, the first reflections as well as multiple, higher-order reflections. When the structural damping is significant and the divergence of the wave is considered, it is reasonable to assume that those waves reflected more than once only contribute marginally to the total field, and thus can be ignored to a first approximation. This suggests a simple description of the field which takes the direct wave and the first reflections into account. The semi-infinite system realizes an example of such models. The question addressed herein is the performance of those simple descriptions. This contribution first presents an investigation of the performance for onedimensional systems. It is shown that the corresponding semi-infinite system can be used to: (1) predict the nodal lines in the spatial domain and the locations of deep troughs in the frequency domain; (2) obtain accurate motion transmissibilities. Second, the extrapolation to two-dimensional systems is presented, accompanied by results for platelike structures.

\section{$11: 35$}

5aSA8. Estimating the vibration energy of an elastic structure via the input impedance. Yuri I. Bobrovnitskii (Lab. of Structural Acoust., Mech. Eng. Res. Inst. of the Russian Acad. of Sci., M. Kharitonievsky Str., 4, 101830 Moscow, Russia,imash@space.ru)

The following problem is posed and solved: given an elastic structure vibrating under the action of a harmonic point force, find the time-average potential and kinetic energy, loss factor, and other energy characteristics of the structure if known are only the complex amplitudes of the force and velocity response at the driving point, $f$ and $v$. The structure is assumed to be linear, with viscous and hysteric damping not necessarily proportional. It is shown that the problem has a mathematically exact solution only for lossless systems. For structures with losses, there exist rather accurate estimates valid at low and middle frequencies. The main result is the equation relating the total vibration energy of the structure to the derivative of its input impedance, $f / v$, or mobility, $v / f$, with respect to frequency. Computer simulation examples with typical structures (rod, plate) illustrate the accuracy and the range of validity of the estimates. The results presented allow one to obtain the energy characteristics of a forced vibrating structure by the most economic way: without measuring or computing the response all over the structure and using only the data measured at one singular point. 
the original discrete force. This replacement is approximately integrated following the Green's function formalism modified by a Taylor expansion

5aSA9. Improving convergence in scattering problems by smoothing discrete constraints using an approximate convolution with a smoothed composite Green's function. Rickard C. Loftman and Donald B. Bliss (Mech. Eng. and Mater. Sci., Duke Univ., Box 90300, Durham, NC 27708)

A method for efficiently treating discrete structural constraints using an approximate Green's function approach to smooth the constraint influence is presented. A method called analytical/numerical matching is used to formulate a composite Green's function that captures the local highresolution content associated with the discrete force in a separate analytic local solution. This local solution in turn implies a smooth replacement for of the forcing strength function. A smooth replacement for the discrete constraint results. This new smoothed problem converges more rapidly than the original discrete one. However, the difference between the smooth solution and the original is retained in the analytic local solution. The result is a composite solution of the original system that is more accurate for a given computational resolution compared with treating the discrete constraint influence directly. In the present example, a modal method is used. Therefore, a given computational resolution is measured by number of modes calculated. The method is demonstrated in the case of beam incident scattering from an infinite thin elastic shell periodically constrained by rings. [Work supported by ONR.]

FRIDAY MORNING, 26 JUNE 1998

FIFTH AVENUE ROOM (W), 8:15 TO 10:45 A.M.

\title{
Session 5aSC
}

\section{Speech Communication: Production and Other Topics}

\author{
Ingo R. Titze, Chair \\ Speech Pathology and Audiology, University of Iowa, 330 WJSHC, Iowa City, Iowa 52242-1012
}

\section{Contributed Papers}

\section{8:15}

5aSC1. Vocal tract shape estimation using three noninvasive transducers. Russel Long, Brad Story, and Ingo Titze (WJ Gould Voice Res. Ctr., Denver Ctr. for the Performing Arts, 1245 Champa St., Denver, CO 80204)

Sentence-level speech simulation requires a description of vocal tract shape parameters as a function of time. Vowel-like configurations can often be derived from a microphone signal; however, the influence of consonants may be more easily detected with the use of additional signals. The electroglottograph (EGG) and an articulatory transducer [McGarr and Lofqvist, J. Acoust. Soc. Am. 72, 34-42 (1982)] are employed to increase the accuracy of vocal tract shape estimation. The microphone signal is analyzed on a frame-by-frame basis for spectral features, energy, and zerocrossing rate. The EGG provides information regarding voicing onset and offset, and the articulatory signal is used to indicate closure and lip rounding. These data are combined to select an appropriate area function for each frame of the sentence. The resulting temporal map of area functions is interpolated and sent to a wave reflection simulator to reconstruct the speech. [Work supported by NIH Grant No. DCO2532.]

\section{8:30}

5aSC2. Simulation of sentence-level speech based on measured vocal tract area functions. Brad Story, Ingo Titze, and Russel Long (WJ Gould Voice Res. Ctr., Denver Ctr. for the Performing Arts, 1245 Champa St., Denver, CO 80204)

An inventory of vocal tract area functions, acquired from magnetic resonance imaging experiments, is used as the basis for creating simulations of sentence-level speech. A first approach is a "brute-force" method in which a phonetic transcription of a sentence is time aligned with the recorded acoustic signal. Each element of the transcription is assigned one of the stored vocal tract area functions in the acquired inventory, thus serving as a "target" through which the time-varying area function should pass. This method has produced intelligible speech but maintains a machinelike quality. A second approach uses a parametric representation of the MRI-acquired area function inventory based on a principal components analysis to generate area functions not in the original inventory. The first three formants of each generated area function were computed and stored in a database, thus creating a mapping between formant frequencies and vocal tract parameters (area functions). Except for the edge of the formant space, the mapping between formants and area functions is oneto-one. This method produces connected speech with natural sounding vowels when only a microphone signal is used but requires additional signals to derive the consonantal contribution to the vocal tract shape. [Supported by NIH grant RO1 DC02532.]

\section{$8: 45$}

5aSC3. Effects of naturally occurring external loads on jaw movements during speech. Douglas M. Shiller, Paul L. Gribble, and David J. Ostry (McGill Univ., Montreal, QC, Canada, ostry@motion.psych.mcgill.ca)

The achieved positions of speech articulators are determined by a combination of intrinsic forces due to muscles and extrinsic forces due to gravity and to head and body motion. If not compensated for, changes in these extrinsic forces will affect articulator positions in behaviors such as speech. In this paper, the extent to which speakers adjust control signals to jaw muscles to compensate for the effects on the jaw due to changes in speaker orientation relative to gravity is assessed. Whether control signals are adjusted to compensate for loads arising during locomotion is also assessed. In one study, subjects produce speech utterances where head and body orientation relative to gravity are varied. In another study, subjects produce speech sequences during locomotion such that the timing of the step cycle applies the maximum load force during either the consonant related (jaw elevated) or vowel related (jaw lowered) phase of movement. The empirically observed patterns of jaw motion are compared with predictions of a model of jaw and hyoid motion which includes muscle properties, dynamics, and modeled neural signals. The hypothesis that during speech, control signals to jaw muscles remain unchanged with variation in these extrinsic loads is specifically tested.

\section{9:00}

5aSC4. Contrasting chest and falsettolike vibration patterns of the vocal folds. David A. Berry and Douglas W. Montequin (Univ. of Iowa, Dept. of Speech Pathol. and Audiol., 334 WJSHC, Iowa City, IA 52242)

Using excised larynges, a hemilarynx methodology was used in order to view vocal fold vibrations from a medial aspect. This was accomplished by removing one fold, inserting a glass plate at the glottal midline, and 
mounting the remaining fold against the glass plate. A stereoscopic imaging system was used to perform 3D tracking of small markers (microsutures) placed on the folds. Chest and falsettolike vibrations patterns were imaged and quantified. These two vibrations patterns will be contrasted with each other, and discussed in terms of previous qualitative comparisons of these phonation types in the literature. [Work supported by Grant No. R29 DC03072 from the National Institute on Deafness and Other Communication Disorders.]

\section{9:15-9:30 Break}

\section{9:30}

5aSC5. The effect of shear thinning in vocal fold tissues-or why we can phonate over two octaves in pitch. Ingo Titze and Roger Chan (Dept. of Speech Pathol. and Audiol. and The Natl. Ctr. for Voice and Speech, Univ. of Iowa, Iowa City, IA 52242)

The shear modulus and viscosity of vocal fold tissues was measured with a rheometer in the frequency range of 1-30 Hz. Extrapolation to phonation frequencies was possible. As in most biological tissues (and for polymers in general), the shear modulus was found to be roughly constant and the viscosity varied inversely with frequency. This makes the damping ratio only a weak function of frequency in phonation. Since selfoscillation of the vocal folds is critically dependent on this damping ratio, it is reasoned that large $F 0$ ranges (on the order of two octaves) are possible only because of this decrease in viscosity, an effect known as shear thinning. A molecular interpretation is given for this effect. [Work supported by NIDCD, Grant No. P60 DC00976.]

\section{$9: 45$}

5aSC6. Pressure-flow relationship in a biophysical model of phonation. Fariborz Alipour and Ingo Titze (Dept. of Speech Pathol. and Audiol., The Univ. of Iowa, Iowa City, IA 52242)

Simulation data are presented from a computer model that combines vocal fold tissue mechanics, laryngeal aerodynamics, and vocal tract acoustics. These simulations are based upon the laws of physics that govern airflow, vibration of the vocal folds, and wave propagation in the vocal tract. The tissue mechanics was modeled with the finite element method with 100 nodes and 166 elements in each layer of the 15-layer model. The laryngeal aerodynamics was modeled by the solution of two-dimensional unsteady Navier-Stokes equations with a finite volume method with a 64 $\times 82$ nonuniform staggered grid. The model has been used to demonstrate self-oscillatory characteristics of the vocal folds and the flow pattern in the larynx. In this study, simulations were performed for lung pressure ranging from 4 to $24 \mathrm{~cm}$ of water. Adduction control was simulated with activation of thyroarytenoid (TA) muscle that caused increased tension and bulging of the vocal folds. Results are reported for glottal waveform, nodal coordinates, and velocity distributions in the larynx. Preliminary data suggest that the relationship between mean pressure and mean flow is almost linear. Frequency and amplitude contours of the model as functions of the lung's pressure and TA activation level reveal their increasing pattern with these parameters.

\section{0:00}

5aSC7. Perception of coarticulated vowels by prelingual infants: Formant transitions specify vowel identity. Ocke-Schwen Bohn (Engelsk Inst., Aarhus Univ., DK-8000 Aarhus C, Denmark, engosb@hum.aau.dk) and Linda Polka (School of Commun. Sci. and Disord., McGill Univ., Montreal, Canada)

German-learning infants were tested for discrimination of four German vowel contrasts in the Silent Center paradigm to examine which acoustic properties of coarticulated vowels (target-spectral, dynamic-spectral) define vowel identity in prelingual infants. Acoustic analyses of naturally produced original syllables $(/ \mathrm{dVt} /$ with $\mathrm{V}=/ \mathrm{i}$, e, I, E, o, U/) revealed no vowel inherent spectral change but only formant movement associated with the gestures for the initial and final consonants. The original syllables were electronically modified to obtain test syllables which contained target-spectral information (the vowel nucleus only), only initial or only final formant transitions, or initial and final transitions in their appropriate temporal relationship, with the vowel nucleus attenuated to silence. Four groups of ten infants each (aged 7-11 months) were tested in the conditioned headturn procedure for discrimination of the test syllables. Each group was tested on one of four contrasts (/i/-/e/, /e/-/I/, /E/-/I/, /o/-/U/) after they had discriminated that contrast successfully in the unmodified condition. Results indicate that target spectral information is not needed to discriminate native contrasts; rather, dynamic spectral information spread over syllable onsets and offsets is sufficient to specify vowel identity in infants. [Work supported by grants of the Deutsche Forschungsgemeinschaft to O.-S. Bohn.]

\section{0:15}

5aSC8. Some observations of the tense-lax distinction theory with reference to English and Korean. Dae W. Kim (Dept. of English, College of Education, Pusan Natl. Univ., Gumjong-Gu, Pusan 609-735, South Korea,dwokim@hyowon.cc.pusan.ac.kr)

A series of experiments with 13 subjects in total and with /VCV/, $/ \mathrm{CVC} /, / \mathrm{CVCVCV} /$, and /CVCVC/ words, using various techniques, was undertaken to verify the hypothesis that the speaker may select at least one of muscles involved in the articulation of a phoneme so that either timing or amplitude variable and/or both of the selected muscle(s) could distinguish /p/ from /b/ in English and /ph, p'/ from /p/ in Korean. The English $/ \mathrm{p} /$ and the Korean aspirated $/ \mathrm{ph} /$ and unaspirated $/ \mathrm{p}^{\prime} /$ were tense, and the English /b/ and the Korean unaspirated /p/ lax. Findings: (1) The EMG data, obtained from the orbicularis oris superior muscle and the depressor anguli oris muscle, supported the tense-lax opposition, except for one English subject in stressed syllables; (2) The leveled peak intraoral airpressure, an output of the synchronized respiratory muscle activities during bilabial stops, backed up the tense-lax distinction, except for one English subject in unstressed syllables, and there was intermuscle compensation between the labial muscle activities and the respiratory muscle activities; (3) The data of the maximum lingual contact in alveolar stops also supported the tense-lax distinction theory. Thus it can be said that the hypothesis has been verified in both languages.

\section{$10: 30$}

5aSC9. Speech intelligibility is highly tolerant of cross-channel spectral asynchrony. Steven Greenberg (Intl. Comput. Sci. Inst., 1947 Center St., Berkeley, CA 94704, steveng@icsi.berkeley.edu) and Takayuki Arai (Intl. Comput. Sci. Inst., Berkeley, CA)

A detailed auditory analysis of the short-term acoustic spectrum is generally considered essential for understanding spoken language. This assumption is called into question by the results of an experiment in which the spectrum of spoken sentences (from the TIMIT corpus) was partitioned into quarter-octave channels and the onset of each channel shifted in time relative to the others so as to desynchronize spectral information across the frequency plane. Intelligibility of sentential material (as measured in terms of word accuracy) is unaffected by a (maximum) onset jitter of $80 \mathrm{~ms}$ or less and remains high $(>75 \%)$ even for jitter intervals of 140 ms. Only when the jitter imposed across channels exceeds $220 \mathrm{~ms}$ does intelligibility fall below $50 \%$. These results imply that the cues required to understand spoken language are not optimally specified in the short-term spectral domain, but may rather be based on some other set of representational cues such as the modulation spectrogram [S. Greenberg and B. Kingsbury, Proc. IEEE ICASSP (1997), pp. 1647-1650]. Consistent with this hypothesis is the fact that intelligibility (as a function of onset-jitter interval) is highly correlated with the magnitude of the modulation spectrum between 3 and $8 \mathrm{~Hz}$. 


\title{
Session 5aSP
}

\section{Signal Processing in Acoustics: Signal Processing for Medical Ultrasound I}

\author{
Stergios Stergiopoulos, Chair \\ DCIEM, P.O. Box 2000, North York, Ontario M3M 3B9, Canada
}

Chair's Introduction-9:15

Invited Papers

9:20

5aSP1. Beyond current medical ultrasonic imaging: Opportunities for advanced signal processing. James G. Miller (Dept. of Phys., Washington Univ., St. Louis, MO 63105)

This keynote address will illustrate some aspects of current medical ultrasonic imaging and attempt to identify areas in which advanced signal processing may be able to contribute to the enhancement and extension of clinical ultrasound. Examples will be drawn primarily from echocardiography, in part because of challenges specifically associated with imaging the beating heart. The presentation will address the goals and needs of the (medical) user in order to avoid approaches which are technically elegant but clinically irrelevant. One feature peculiar to medical imaging, which limits the effective resolution to far less than the expected theoretical limit for the few hundred micrometer wavelength imaging systems in current use, is the subtle variation of elastic properties of soft tissue and the corresponding local variation in the speed of sound. Both random and systematic (for example, anisotropic) variations contribute to degradation in image quality, including the effect known as speckle. Opportunities for advanced signal processing may include not only approaches designed to enhance image resolution but also contributions to areas such as contrast agents and tissue characterization. The talk is designed to provide a broad overview which might serve as a common reference point for subsequent presentations.

\section{0:00}

5aSP2. The evolution of medical ultrasonic imaging systems. John M. Reid (Consultant 16711254 Ave. SE, Issaquah, WA 98027)

A limit to effective soft tissue imaging is set by the acoustic properties of tissue. The attenuation is roughly proportional, and the size of a resolution element inversely proportional to frequency. Thus the number of resolution elements per image is fixed. Accordingly, acoustic frequencies from about 1 to $50 \mathrm{MHz}$ are required. Effective imaging has required the development and application of innovative high-frequency hardware; particularly the transducers. These have progressed from single element types to 512 element arrays in production, with two-dimensional arrays having 1282 elements in development. The electronics now require many more channels with a dynamic range exceeding $100 \mathrm{~dB}$ and, with the introduction of digital methods, even multiple very fast A/D converters in the beamformer. Display devices have gone from WWII CRT's with 200 spots per radius to digital image stores on CRT's with thousands of spots available. These can now do image processing with software. The earlier systems survive in some applications that require them. The single element types are still used in high-frequency catheters, for example. The wideband transducers have opened up new methods, enhanced Doppler, contrast and tissue imaging that use a wider range of frequencies than previously possible. The single element types are still used in high-frequency catheters, for example.

10:30-10:40 Break

\section{0:40}

5aSP3. 3-D ultrasound imaging of the prostate. Aaron Fenster and Donal Downey (Robarts Res. Inst., London, ON N6A 5K8, Canada)

An important aspect that needs improvement in medical ultrasound systems is related to the 2-D imaging of the prostate. 2-D viewing of 3-D anatomy limits our ability to quantify and visualize prostate disease and is partly responsible for the reported variabilities. This occurs because: (i) the diagnostician must integrate multiple 2-D images in his mind during the procedure, leading to inefficiency and variability; (ii) The 2-D ultrasound images represent a thin plane at some arbitrary angle in the body, making it difficult to localize the image plane. To overcome these difficulties, we have developed a 3-D ultrasound system to image the prostate. Our 3-D ultrasound imaging system consists of: a conventional ultrasound machine and transducer; a custom-built assembly for rotating the probe under microcomputer control; a microcomputer with a video grabber; and software to reconstruct and display 3-D images. A typical scan of 200 2-D B-mode images takes only $13 \mathrm{~s}$, and the reconstruction less than $1 \mathrm{~s}$. This paper will detail the 3-D imaging approach and its use for imaging the prostate in 3-D. Various applications will be discussed related to prostate cancer diagnosis, prostate volume measurements, and 3-D ultrasound-guided therapeutic procedures such as cryosurgery. 
5aSP4. The analysis and classification of small-scale tissue structures using the generalized spectrum. Kevin D. Donohue (Dept. of Elec. Eng., Univ. of Kentucky, Lexington, KY 40506), Flemming Forsberg, and Ethan J. Halpern (Thomas Jefferson Univ. Hospital, Philadelphia, PA 19107)

Conventional ultrasonic imaging systems primarily use backscattered signals for creating qualitative images that reveal large-scale structures, such as tissue boundaries. Efforts to extract additional quantitative information have resulted in limited success. The nonstationarities of the scatterers comprising biological tissue often violate conditions for applying common signal characterization and estimation methods. In addition, ultrasonic tissue properties (such as attenuation, velocity, scatterer density, size, and structure) ambiguously encode information into the backscattered signal, making it difficult or impossible to extract and quantify a single property. This paper presents the generalized spectrum (GS) as a method for analyzing and quantifying the properties of small-scale resolvable structures (on the order of 1 to $4 \mathrm{~mm}$ ), which result from tissue structures such as lobules, ducts, and vessels. The GS extends the capabilities of power spectral density to include meaningful phase information that results from small-scale structure. The relevant properties of the GS include its ability to reduce the effects' diffuse scatterers (speckle), and permit normalization schemes that significantly limit the effects of system response and attenuation from the overlying tissue. An implementation of the GS in an analysis and classification of normal and metastic liver tissues is also described.

\section{1:40}

5aSP5. A Wold decomposition-based autonomous system for detecting breast lesions in ultrasound images of the breast. Georgia Georgiou and Fernand S. Cohen (Imaging and Comput. Vision Ctr., Dept. of Elec. and Comput. Eng., Philadelphia, PA 19104)

This paper presents an autonomous system for detecting lesions in the breast. The Wold decomposition algorithm described is used to decompose the RF echo of the breast into its diffuse and coherent components. The coherent component is modeled as a periodic sequence and the diffuse component is modeled as an autoregressive time series of low order. The parameters of the model are estimated from selected regions of the RF image and used as detection features. The database of images that was used contained 370 B-scan images from 52 patients, obtained in the Radiology department of the Thomas Jefferson Hospital. The pathologies of interest are carcinoma fibrocystic and stromal fibrosis disease and fibroadenoma. Empirical ROC techniques were used to evaluate the detection rate on single parameters of the model, such as the residual error variance and the autoregressive parameters of the diffuse component of the RF echo. The area under the empirical ROC curve for detecting lesion regions versus normal RF regions is 0.901 . The area under the ROC curve for detecting carcinoma versus normal regions is 0.904 . The corresponding areas for normal regions versus stromal fibrosis/fibrocystic regions and fibroadenoma regions are 0.942 and 0.899 , respectively.

\section{Contributed Papers}

12:10

5aSP6. Tracking and correcting for organ motion artifacts in ultrasound tomography systems. Amar C. Dhanantwari (Dept. of Elec. and Comput. Eng., Univ. of Western Ontario, London, ON N6A 5B9, Canada) and Stergios Stergiopoulos (Defence and Civil Inst. of Environ. Medicine, P.O. Box 2000, North York, ON M3M 3B9, Canada)

Motion artifacts have been identified as a problem in medical tomography systems. This problem, however, is well known in other types of real-time imaging systems such as radar satellites and sonars. In this case it has been found that the application of an overlap processing scheme [J. Acoust. Soc. Am. 86, 158-171 (1989)] increases the resolution of a phased array imaging system and corrects for motion artifacts as well. Reported results have shown that the problem of correcting motion artifacts in synthetic aperture applications is centered on the estimation of a phase correction factor. This correction factor is then used to compensate for the temporal phase differences between sequential sensor-array measurements in order to synthesize the spatial information coherently. This paper describes an approach which tracks the organ motion and allows the artifacts to be isolated in ultrasound tomography systems. Two sources are utilized so that two sets of projections are generated that are identical in space but separated in time. Then the spatial overlap correlator processing scheme is used to synthesize the 2-D projection data coherently. This provides the desired phase correction factor, which compensates for the phase fluctuations caused by the subject's organ-motion effects and tracks the organ motion.
$12: 30$

5aSP7. Wavefront distortion measurements in the human breast. $R$. C. Gauss, G. E. Trahey (Dept. of Biomed. Eng., Duke Univ., 136 Eng. Bldg., Durham, NC 27708, rgauss@acpub.duke.edu), and M. S. Soo (Duke Univ. Medical Ctr., Durham, NC 27710)

Published wavefront distortion (phase aberration) measurements for the human breast have been inconsistent, ranging from mild phase aberrations $(8 \mathrm{~ns} \mathrm{rms})$ to severe phase aberrations $(67 \mathrm{~ns} \mathrm{rms})$. These measurements are required to specify arrays and assess the potentials of and appropriate algorithms for adaptive imaging. They require high interelement uniformity so that receive signal variations between elements can be attributed to wave propagation effects. Array elements must be small to minimize the integration of the arriving wavefront across the face of the element. The array aperture must be large enough to allow target visualization and to provide high correlations between neighboring elements for pulse-echo measurements. An apparatus to make concurrent pulse-echo and pitch-catch (through transmission) waveform measurements in a clinical setting has been developed. The breast is stabilized between opposing transducers with light compression. After fixing the position of the transducers, pulse-echo and pitch-catch snapshots are captured sequentially with tissue and reference phantom targets. Data were collected from four views of the left breast in 12 volunteers. The rms phase error was significantly smaller for pulse-echo measurements $(25 \pm 14 \mathrm{~ns} \mathrm{rms})$ than for pitch-catch measurements ( $60 \pm 23 \mathrm{~ns} \mathrm{rms})$. [Work supported by NIH.]

\section{2:45-1:15 Panel Discussion}




\title{
Session 5aUW
}

\section{Underwater Acoustics: Bistatic Scattering from Seabed and Surface: Models and Measurements I}

\author{
Paul C. Hines, Chair \\ Defence Research Establishment Atlantic, P.O. Box 1012, Dartmouth, NS B2Y 3Z7, Canada \\ Chair's Introduction-9:15
}

Invited Papers

9:20

5aUW1. A practical model for high-frequency seabed bistatic scattering strength. Darrell R. Jackson (Appl. Phys. Lab., College of Ocean and Fishery Sci., Univ. of Washington, Seattle, WA 98105) and Anatoliy N. Ivakin (Andreev Acoust. Inst., Shvernika 4, Moscow 117036, Russia)

\begin{abstract}
The authors have previously reported a model for bistatic scattering by elastic seabeds. This model is extended here to cases of larger roughness by replacing the rough-interface perturbation approximation with the small-slope approximation [Yang and Broschat, J. Acoust. Soc. Am. 96, 1796-1804 (1994)]. Consistency with older models is shown by comparisons of calculations for silt and sand examples. Correlations between volume fluctuations of different types (e.g., density and compressional wave speed) are considered, and it is shown that the effects of such correlations are more readily observed in bistatic scattering measurements as opposed to backscattering measurements. Finally, the importance of elastic effects in scattering by rocky seabeds is illustrated. [Work supported by ONR.]
\end{abstract}

9:40

5aUW2. High-frequency bistatic scattering from sediments-experiments and modeling. Kevin Williams and Dajun Tang (Appl. Phys. Lab., College of Ocean and Fishery Sci., Univ. of Washington, Seattle, WA 98105, williams@apl.washington.edu)

Typically, for frequencies in the $10-100 \mathrm{kHz}$ range, the interaction of sound with the ocean bottom is a scattering process. Use of bistatic geometries can give insight into the scattering process not possible via traditional monostatic experiments. The trade-off is that such experiments are more difficult and more data are needed to get statistically meaningful results to compare with models. During the last 5 years, the Coastal Benthic Boundary Layer (CBBL) program allowed the opportunity to develop and fine-tune one method of obtaining bistatic data. That evolution will be briefly described and the data from three sites (one mud, one sand, one sand-silt-clay) shown. The sand and sand-silt-clay site data are compared with a model based on surface roughness and volume perturbation scattering. The mud site had methane bubbles embedded within it and the data are compared with a model based on bubble scattering. The physical insight derivable from the model/data comparison in the mud case will be discussed. [Work supported by ONR.]

\section{Contributed Papers}

\section{0:00}

5aUW3. Models of volume and roughness scattering in stratified seabeds. Anatoliy N. Ivakin (Andreev Acoust. Inst., Shvernika 4, Moscow 117036, Russia, aniva@glasnet.ru)

At high frequencies, two mechanisms of seabed scattering are usually taken into account which are due to roughness of the seabed surface (sediment-water interface) and volume inhomogeneities of near-surface sediment. The mean parameters of bottom medium are usually considered as independent from spatial coordinates. This corresponds to the assumption that sound penetration into the sediment is sufficiently small because of strong absorption. In this paper, more general models are considered where seabeds are assumed as statistically stratified (plane-layered on the average) with the mean parameters taken as various functions of depth. In particular, they can be step functions corresponding to different types of sediments in different layers. Another type of stratification corresponds to continuous depth-dependences and involve gradients of mean parameters. Effects of stratification are shown to be important at lower frequences due to scattering from internal interfaces and volume inhomogeneities of deep layers as well as the influence of regular refraction and reflection within seabed medium. As numerical examples, angular dependencies of the scattering strength in both monostatic and bistatic cases at different frequen- cies are calculated for seabeds of various types. Comparison with available data is presented and discussed. [Work supported by ONR.]

\section{0:12}

5aUW4. Bottom volume scattering-Modeling and data analysis. Dan Li, George V. Frisk (Woods Hole Oceanogr. Inst., Woods Hole, MA 02543), and Dajun Tang (Univ. of Washington, Seattle, WA 98105)

As part of the Acoustic Reverberation Special Research Program (ARSRP), low-frequency bottom scattering from a deep ocean sediment pond was measured using an omnidirectional source and a vertical linereceiving array deployed near the bottom. Sediment volume heterogeneities were found to be the major contributor to the measured scattered fields [Tang et al., J. Acoust. Soc. Am. 98, 508-516 (1995)]. Taking advantage of the experimental geometry, a model is developed which can generate realizations of three-dimensional random inhomogeneities in sediments and can simulate scattered fields based on these realizations using a perturbation approach. In this model, the broadband propagator (the Green's function) is obtained using an exact numerical method. 
Therefore, the model can handle any layered sediment environments with scatterers distributed within any of the layers. The model predictions are compared to the experimental data across the available frequency band, and the results are favorable. [Work supported by ONR.]

\section{0:24}

5aUW5. High-frequency bottom backscattering from a homogeneous seabed. Kevin B. Briggs (Marine Geosciences Div., Naval Res. Lab., Stennis Space Center, MS 39529, kbriggs@zephyr.nrlssc.navy.mil) and Steve Stanic (Acoust. Div., Naval Res. Lab., Stennis Space Center, MS 39529)

High-frequency acoustic backscattering was measured from a sandy site located in very shallow water $(8 \mathrm{~m})$ off Tirrenia, Italy, at a range of frequencies and grazing angles. Concomitant measurements of the seabed indicated that the very fine sand was well sorted, relatively uniform with respect to vertical and horizontal distribution of compressional wave velocity and attenuation, and depleted with respect to biological activity. Bottom scattering strengths were very low and variability of sediment parameters was exceptionally low. Coefficients of variation determined for sediment compressional wave velocity and attenuation were $0.29 \%$ and $7.2 \%$, respectively. These data compare with previously recorded minima of $0.30 \%$ and $14.8 \%$ for the same respective parameters, in nearshore, mobile fine sands from the Gulf of Mexico and the Pacific Ocean. Lack of variability in sediment sound velocity and density concurrent with low acoustic bottom scattering strength measurements would endorse recent modeling approaches that predict scattering from sediment impedance fluctuations. Acoustic data are compared with predictions from the composite roughness model (modified for inclusion of sediment volume scattering parameters) at various frequencies and grazing angles in order to test the model's response at minimal perturbation of seabed properties.

\section{0:36}

5aUW6. A porous medium with porosity variations. Nicholas $P$. Chotiros (Appl. Res. Labs., Univ. of Texas, P.O. Box 8029, Austin, TX 78713-8029)

The BiotUs equations of acoustic propagation in porous media have no inherent scattering mechanism, because the pores are modeled as smoothsided tubular passages with constant cross section. They predict two compressional and one shear wave that propagate independently. Real porous media usually have pores with varying cross section, which may be considered as variations in porosity on a microscopic scale. Although any single variation on such a scale would have a negligible effect on the acoustics of the medium, the cumulative effect of a field of such variations is unknown. A one-dimensional structure with varying porosity was modeled numerically to study its properities as an acoustic medium. It was found that, not only does it possess an inherent volume scattering strength, but it also allows energy transfer between the three types of waves as they propagate. [Work supported by ONR 321 OA.]

\section{0:48-11:03 Break}

\section{1:03}

5aUW7. A comparison of bistatic scattering from two geologically distinct mid-ocean ridges. Chin Swee Chia (MIT, 77 Massachusetts Ave., Cambridge, MA 02139) and Laurie T. Fialkowski (Naval Res. Lab., Washington, DC 20375)

A comparison is made of bistatic scattering from two distinct deepocean ridges. The ridges are located along a segment valley on the western flank of the Mid-Atlantic Ridge (MAR). The acoustic data sets were acquired during the Main Acoustics Experiment of the Acoustic Reverberation Special Research Program. Detailed analysis of bistatic scattering from one of these ridges, named $\mathrm{B}^{\prime}$, has been previously presented by some of the authors. The purpose of this paper is to present a similar analysis for the second ridge, $\mathrm{C}^{\prime}$, for comparative purposes. This comparison is important because the ridges span the two geologically distinct classes found in the MAR. There is a possibility, therefore, that their scattering characteristics may be extrapolated to ridges throughout the MAR. For example, B' is an "outside corner" and therefore is composed of highly lineated scarps and terraces, while $\mathrm{C}^{\prime}$ is an 'inside corner" that is characteristically amorphous with irregular large-throw normal faults. While major scarps on these ridges can be easily resolved by the towedarray system, smaller-scale anomalies such as gullies and trellises are often underresolved by nearly an order of magnitude. These anomalies are found to introduce large variances in the bistatic scattering characteristics of a given scarp.

\section{1:15}

5aUW8. Multistatic scattering from anisotropically rough interfaces in horizontally stratified waveguides. Jaiyong Lee (MIT, Cambridge, MA 02139) and Henrik Schmidt (SACLANT Undersea Res. Ctr., La Spezia, Italy)

A model of multistatic scattering and reverberation from an anisotropically rough seabed in shallow water has been developed by combining a scattering theory based the method of small perturbations [W. A. Kuperman and H. Schmidt, J. Acoust. Soc. Am. 79, 1767-1777 (1986)] with a seismo-acoustic propagation model $[\mathrm{H}$. Schmidt and J. Glattetre, J. Acoust. Soc. Am. 78, 2105-2114 (1985)]. This new computational model provides full 3-D wave theory simulations of multistatic rough bottom reverberation in horizontally stratified waveguides. The seabed and subbottom roughnesses are represented by anisotropic roughness patches covering the actual sonar footprint. The scattering theory convolves the incident sonar field with the roughness to provide a distribution of virtual source panels that are then fed into the propagation model to provide a numerically efficient azimuthal Fourier series of wave number integrals for evaluating the 3-D reverberant field. The efficiency of the new hybrid model enables the modeling of 3-D field statistics by Monte Carlo simulation, as well as time-domain field simulations by Fourier synthesis. The model has been verified by comparison with analytical solutions for the simple problem of determining the 3-D multistatic scattering strength of a randomly rough interface separating two fluid half-spaces. Here the new model is used to investigate the dependence of the multistatic reverberant field on environmental parameters such as anisotropic roughness statistics, and bottom stratification and composition. [Work supported by ONR.]

\section{1:27}

5aUW9. Integral equation method for bistatic volume scattering from the seafloor. Christopher D. Jones and Darrell R. Jackson (Appl. Phys. Lab., College of Ocean and Fishery Sci., Univ. of Washington, Seattle, WA 98105)

In most theoretical developments of sediment volume scattering, the medium is assumed to have weak fluctuations in sound speed and density and is assumed to be statistically homogeneous. An exact integral equation method has been developed and is used to test these assumptions. Small perturbation theory results for single frequency bistatic scattering from the seafloor are compared with Monte Carlo simulations. Half-space and multiple scattering effects not included in most previous treatments are observed.

\section{1:39}

5aUW10. Time/frequency spread for bistatic geometries. R. Lee Culver and Christopher J. Link (Appl. Res. Lab., Penn State Univ., P.O. Box 30, State College, PA 16804)

The overall goal of this work is to develop a functional characterization of, and empirical models for, high-frequency, broadband acoustic propagation through a shallow ocean channel. One important aspect of this characterization is the time/frequency spread, which quantifies how much signals spread in time and frequency as they propagate. Because time and frequency spread are random quantities which vary from pulse to pulse, time/frequency spread must be characterized using a second-order statistic, e.g., the scattering function, which is the expected value of the meansquare spreading function. The scattering function has been measured in the following ocean environments: sandy gravel bottom, isovelocity sound 
speed profile (SSP); rough rock bottom, slightly downward-refracting SSP; and mud bottom, upward-refracting SSP. A statistical model for the scattering function is being developed for these environments based upon radial basis function decompositions of the scattering function estimates. The ultimate use of a scattering function model will be with simulation to assess and optimize acoustic receiver performance. [Work is sponsored by the Office of Naval Research Ocean Acoustics Program.]

\section{1:51}

5aUW11. Sound waves attenuation in shallow water with rough boundaries. Boris G. Katsnelson, Sergey A. Pereselkov (Dept. of Phys., Voronezh Univ., 1 University Sq., Voronezh 394693 Voronezh, Russia), Venedict M. Kuz'kin, and Valery G. Petnikov (General Phys. Inst., 137333 Moscow, Russia)

Here the object is to estimate the effect of scattering by randomly rough seabed and surface waves on attenuation of low-frequency sound waves in shallow water. The experiment was pursued with $\mathrm{CW}$ signals $(100$ and $230 \mathrm{~Hz})$ in Barents Sea. The transmission loss and interference pattern of a sound field were measured within $100 \mathrm{~km}$ of the acoustic sources. Mesoscale relief of the bottom with spatial resolution $10 \mathrm{~m}$ and wind parameters were recorded as well. The experimental results (the average sound intensity) were compared with theoretical ones calculated with and without taking into account seabed and surface roughness. The calculation was carried out on the base of modal theory of sound scattering by rough boundaries in a waveguide. It was demonstrated that the random roughness substantially affects the transmission loss for long distances. In this paper the method is suggested for bottom parameter estimation using the statistical attributes of sound field in shallow water with random rough boundaries as well. [The work was supported by Russian Foundation of Basic Research (Grant 96-02-17194).]
5aUW12. Modal theory of sound propagation in a random irregular shallow-water waveguide (numerical calculation and diffusion approach). Boris G. Katsnelson and Sergey A. Pereselkov (Dept. of Phys., Voronezh Univ., 1 University Sq., Voronezh 394693 Voronezh, Russia)

Sound field changing due to scattering by random irregularities in shallow water is considered. Shallow water is a randomly inhomogeneous media bounded by absorbing bottom and surface, which are randomly irregular (rough) as well. Longitudinal scales of all types of irregularities are greater than cross scales. Sound field is the sum of modes with random amplitudes. A system of stochastic differential equations for modal amplitudes is derived. Asymptotic approximation is used to obtain equations for statistical moments of modal amplitudes. The behavior of average modal intensity is described by diffusion equation. Comparative analysis of scattering and absorbing effects on sound propagation is carried out. Scattering leads to two processes: intensity transformation from a coherent (average) field component into a noncoherent (random) one, and intensity redistribution between modes. The next problem is interference structure alteration due to scattering and absorption. This alteration leads in turn to space redistribution of the field intensity. It is shown that for the ranges from the source, a much greater than "automodel range" situation takes place, and that depth dependence of intensity is constant in range. It is the result of equilibrium between two phenomena: modal intensity absorbing and modal intensity redistribution due to scattering. Some examples are considered. [Work was supported by RFBR (Grant 97-05-64878).]
FRIDAY MORNING, 26 JUNE 1998

Session 5aPLb
Plenary Lecture
Thomas D. Rossing, Chair
Department of Physics, Northern Illinois University, DeKalb, Illinois 60115
Chair's Introduction-11:00
Invited Paper

11:05

5aPLb1. Nonlinearity, complexity, and the sounds of musical instruments. Neville H. Fletcher (Res. School of Physical Sci., Australian Natl. Univ., Canberra 0200, Australia, neville.fletcher@anu.edu.au)

\footnotetext{
Musical instruments can be treated to a first approximation as linear harmonic systems, but closer examination shows that they are, almost without exception, nonlinear and inharmonic. Sustained-tone instruments such as violins and clarinets can be characterized as "essentially nonlinear" and owe their deceptively simple behavior to mode locking due to strong nonlinearity. When the nonlinearity is weakened, the behavior becomes complex, with inharmonic and multiphonic sounds that are sometimes exploited in modern music. In contrast, impulsively excited instruments, such as guitars, pianos, gongs, and cymbals, may be described as "incidentally nonlinear." They would function quite acceptably if the nonlinearity were to be eliminated but, when it becomes strong, they exhibit physically and aurally interesting behavior such as period doubling, frequency multiplication cascades, and chaotic oscillation. This paper will explore some of these phenomena and demonstrate both their physical origins and musical utility.
} 


\title{
Session 5pAAa
}

\section{Architectural Acoustics: Classroom Acoustics}

\author{
Gary W. Siebein, Chair \\ Department of Architecture, University of Florida, 231 Arch, P.O. Box 115702, Gainesville, Florida 32611-5702
}

Chair's Introduction-1:30

\section{Contributed Papers}

$1: 35$

5pAAa1. Classroom acoustics I: The acoustical learning environment: Participatory action research in classrooms. Mary Jo Hasell, Philip Abbott, Gary W. Siebein, Martin A. Gold, Hee Won Lee, Mitchell Lehde, John Ashby, Michael Ermann, and Carl C. Crandell (Architecture Technol. Res. Ctr., 336 ARCH, Univ. of Florida, P.O. Box 115702, Gainesville, FL 32611-5702)

This pilot study used participatory fieldwork in a number of kindergarten through eighth grade classrooms to evaluate the acoustic setting that supports learning. Dynamic interactions among administrators, teachers, students, parents, and research team members makes a difference in learning. It was found that classroom interaction depends on the social make-up, anticipated behavior, intellectual level, teaching method and theories, and hearing capacity of participants, as well as the physical characteristics of the classrooms. Participatory action research (PAR) resulted in the development of appropriate classroom observation tools utilizing checklists, summary scales, and hierarchial rating scales. The learning climate, lesson clarity, instructional variety, task orientation, and student engagement were used to identify variables for acoustical studies. The PAR was instrumental in determining the protocol for the acoustical measurement studies described in Classroom Acoustics II. Field observations began with an understanding of the adaptive behaviors of the classroom participants such as how the teachers modified their location and sometimes even the furniture to maintain short speaker to listener distances. The findings indicate a range of solutions are needed to improve the acoustical learning environment including innovative teaching methods, improving room acoustical design, reducing background noise levels, and, in some cases, using amplification systems.

\section{1:50}

5pAAa2. Classroom acoustics II: Acoustical conditions in elementary school classrooms. Martin A. Gold, Hee Won Lee, Gary W. Siebein, Mitchell Lehde, John Ashby, Michael Ermann, Mary Jo Hasell, Philip Abbott, and Carl C. Crandell (Architecture Technol. Res. Ctr., 231 ARCH, Univ. of Florida, P.O. Box 115702, Gainesville, FL 32611-5702)

Acoustical measurements of speech transmission index, reverberation time, early reverberation time, early-to-late energy ratios, loudness (or relative strength), articulation index, background noise levels, and signalto-noise ratios were made in a number of elementary school classrooms in one school district to see how many rooms actually had acceptable acoustical conditions. The source and receiver locations for the acoustical measurements were determined through the participatory action research (PAR) described in Classroom Acoustics I. Measurements were made using a TEF analyzer with custom software to compute additional acoustical measurements. Both omni-directional and directional loudspeakers were used for the source signals. The acoustical measurements were conducted in a variety of source receiver conditions as observed in the PAR studies in the classrooms. General observations are related regarding the relationship between the measurements and the PAR. For example, the STI was always greater than 0.75 for the conditions under which the teachers actually taught. Speaker-to-listener distances of $4 \mathrm{~m}$ or less were observed for many classroom activities. Likewise the teachers employed many creative ways to control the behavior of the students to reduce background noise levels while they actually spoke.

\section{2:05}

5pAAa3. Classroom acoustics III: Acoustical model studies of elementary school classrooms. Gary W. Siebein, Mitchell Lehde, Hee Won Lee, John Ashby, Michael Ermann, Martin A. Gold, Mary Jo Hasell, Philip Abbott, and Carl C. Crandell (Architecture Technol. Res. Ctr., 231 ARCH, Univ. of Florida, P.O. Box 115702, Gainesville, FL 32611-5702)

A 1:4 scale model was constructed with a series of interchangeable wall and ceiling panels to allow a battery of acoustical tests to be conducted for a variety of classroom designs. The walls and ceiling of the room could be changed from sound absorbent to sound reflective materials quickly. Scale furniture was also constructed for the room. The walls of the model could be adjusted from approximately 7 to 10 meters in length and from $2.5 \mathrm{~m}$ in height to $7 \mathrm{~m}$ in height. Acoustical measurements of speech transmission index, reverberation time, early reverberation time, early-to-late energy ratios, loudness (or relative strength), and articulation index were made in a number of simulated elementary school classrooms using both a TEF analyzer and impulse spark techniques. Classrooms where field measurements were taken as described in Classroom Acoustics II were modified to attempt to improve acoustical conditions in the rooms. The location and amount of absorbent material, the location and amount of sound reflective material, and the room volume were the major variables considered. In general rooms that followed good architectural acoustics design principles produced favorable acoustical measurements.

2:20

5pAAa4. Classroom acoustics IV: Speech perception of normalhearing and hearing-impaired children in classrooms. Carl $\mathrm{C}$. Crandell, Gary W. Siebein, Martin A. Gold, Mary Jo Hasell, Philip Abbott, Mitchell Lehde, and Hee Won Lee (Architecture Technol. Res. Ctr., 231 ARCH, Univ. of Florida, P.O. Box 115702, Gainesville, FL 32611-5702)

The present investigation examined the speech-perception abilities of normal-hearing and hearing-impaired children in a number of classroom environments. Speech perception was assessed as a function of teacher location, background noise levels, early sound reflections, reverberation time, and signal-to-noise ratio. Specific acoustical measurement procedures are described in Classroom Acoustics I and II. Speech perception was assessed by nonsense syllables, monosyllabic words, and sentences. Normal-hearing populations included children, aged 5-17 years, who are progressing normally in school; learning-disabled children; children with central auditory processing deficits; articulatory- and/or languagedisordered children; children with developmental delays and/or attention deficits; and children for whom English is a second language. Hearingimpaired populations consisted of children with minimal-to-severe degrees of bilateral and unilateral, sensorineural or conductive hearing loss. Data will be discussed in view of developing appropriate classroom acoustics for normal-hearing and hearing-impaired pediatric listeners. 
(and class sessions held in the classrooms) are being systematically mea-

5pAAa5. Assessing speech intelligibility in classrooms at the University of Washington. Dean Heerwagen (Dept. of Architecture, Univ. of Washington, Box 355720, Seattle, WA 98195-5720) and Tarik Khiati (Dept. of Urban Design and Planning, Univ. of Washington)

The University's Committee on Accessibility has initiated an investigation of classroom acoustical conditions. This investigation has been undertaken in response to complaints made by University faculty members who state that they are experiencing difficulties in understanding student's speech during class sessions, when students ask questions or engage in discussion. A survey of University faculty identified 75 problematic classrooms (out of the $300+$ classrooms on the University campus). A sample of generally representative classrooms has been selected: these classrooms sured to determine their acoustical properties. Seven parameters have been anticipated as the causes of inferior speech intelligibility: to strong background noise levels, too weak signal-to-noise ratios, excessive reverberation, too great speak-to-listener distances, various speaker idiosyncrasies (e.g., accenting, mumbling, rapidity, using unfamiliar verbiage, and so forth), acoustical defects of the classroom, and faulty amplification systems. To date, measurements show that the principal acoustical faults causing these intelligibility difficulties are high background noise levels, inadequate amounts and locations of absorptive materials and assemblies, and student speech characteristics including too-long distances separating faculty and students. In selected classrooms corrections are being undertaken.

FRIDAY AFTERNOON, 26 JUNE 1998

GRAND FOYER (W), 1:00 TO 5:00 P.M.

\title{
Session 5pAAb
}

\section{Architectural Acoustics: Room Acoustics Measurement Techniques, Absorption and Diffusion (Poster Session)}

\author{
Julie Wiebusch, Cochair \\ Greenbusch Group, 919 NE 71st Street, Seattle, Washington 98115-5634 \\ John Greenlaw, Cochair \\ Greenbusch Group, 919 NE 71st Street, Seattle, Washington 98115-5634
}

\section{Contributed Papers}

\begin{abstract}
All posters will be on display from 1:00 p.m. to 5:00 p.m. To allow contributors an opportunity to see other posters, contributors of odd-numbered papers will be at their posters from 1:00 p.m. to 3:00 p.m. and contributors of even-numbered papers will be at their posters from 3:00 p.m. to 5:00 p.m.
\end{abstract}

5pAAb1. Characterizing scattering from room surfaces. Tristan J. Hargreaves, Trevor J. Cox, Y. W. Lam (Dept. of Acoust. and Audio Eng., Univ. of Salford, Salford M5 4WT, UK), and Peter D'Antonio (RPG Diffusor Systems, Inc., Upper Marlboro, MD 20772)

There is a need to characterize the scattering from room surfaces in terms of a diffusion parameter. Such a parameter is required for many geometric room acoustic models and to enable the relative diffusion performance of surfaces to be evaluated. In addition, it is hoped that the characterization of scattering will aid designers of rooms. This paper examines the derivation of diffusion parameters from the distribution of scattered energy, which is one possible route to characterizing surface scattering. Previous investigations have concentrated on obtaining diffusion parameters from two-dimensional polar distributions-either measurements or predictions using BEM techniques. The advantages and disadvantages of these parameters are outlined and an alternative parameter of this type which overcomes some of the disadvantages is described. A new capability to perform measurements in three dimensions, which has broadened the applicability of the results from this study, is described. Extending the use of existing two-dimensional diffusion parameters to characterize hemispherical scattering will be discussed. Some solutions to the problems which arise when using these parameters to characterize scattering from large surfaces or those mounted in baffles will be presented.

5pAAb2. Numerical study on sound absorption characteristics of brick/block absorbing walls. Shinichi Sakamoto, Dong-Jun Joe, Hideki Tachibana (Inst. of Industrial Sci., Univ. of Tokyo, 7-22-1 Roppongi, Minato-ku, Tokyo, 106 Japan), and Hikari Mukai (Ono Sokki Co., Ltd., 1-16-1 Hakusan, Midori-ku, Yokohama, 226 Japan)

The brick/block absorbing walls with openings and backing air space with porous materials are often used for absorption in low frequencies. The absorption in low frequencies can be explained by the well-known theory of Helmholtz resonance. In some measurement results of absorption coefficient, however, high absorption coefficients are seen in high frequencies. Regarding this problem, the authors have been investigating theoretically and experimentally as presented at the last ASA meeting (at Penn. State). Following these studies, further numerical analysis was carried out on simple 2-D models. The wave phenomena in the cavity and backing space/material were formulated by combining the FEM with the 
scattering wave theory. Comparison between the calculated result and the result of a 1/10 scale model experiment has indicated the validity of the numerical analysis.

5pAAb3. Absorption characteristics of a double-leaf membrane with an absorptive layer in its cavity. Kimihiro Sakagami, Toru Uyama (Environ. Acoust. Lab., Faculty of Eng., Kobe Univ., Rokko, Nada, Kobe, 657 Japan), Masakazu Kiyama (Kobe Univ., Rokko, Nada, Kobe, 657 Japan), and Masayuki Morimoto (Kobe Univ., Rokko, Nada, Kobe, 657 Japan)

Absorption characteristics of double-leaf membranes with an absorptive layer in the cavity are theoretically analyzed. The double-leaf membrane is modeled as two infinite membranes with a cavity between them, which consists of three layers of arbitrary media. The theory also includes the effect of permeability of the interior leaf. The effects of parameters of the absorptive layer on the absorption characteristics are discussed through numerical examples. The absorption characteristics of an impermeable double-leaf membrane are characterized by a mass-spring resonance peak at low frequencies. This peak becomes larger and shifts to lower frequencies as the thickness of the absorptive layer increases. The absorption characteristics are, in permeable cases, similar to those of the conventional porous absorbents; the absorption is low at low frequencies and higher at high frequencies. As the thickness of the absorptive layer increases, the absorption becomes higher at all frequencies. The change in position of the absorptive layer affects the absorption characteristics of the doubleleaf membrane. This effect is related to the standing-wave sound field in the cavity. The flow resistance of the absorptive layer has an optimum value to maximize the absorption of a double-leaf membrane.

5pAAb4. Numerical simulations of the modified Schroeder diffuser structure. Antti Jrvinen, Lauri Savioja (Helsinki Univ. of Technol., Lab. of Acoust. and Audio Signal Processing, P.O. Box 3000, FIN-02015 HUT, Finland), and Kaarina Melkas (Nokia Res. Ctr., FIN-33721 Tampere, Finland)

Schroeder diffusers are widely used in architectural acoustics because they offer unique diffusion characteristics. It has been recently shown that the Schroeder diffusers are also efficient low-frequency absorbers. In this paper a new modified Schroeder diffuser structure is presented. The new folded structure allows one to extend the low-frequency limit of the Schroeder diffuser so that the cutoff frequency is not directly proportional to the depth of the structure. Other important advantage of the new structure is that the total volume of the structure is fully utilized as a diffuser. The modified structure is also easier to manufacture than the traditional Schroeder diffusers. The modified structure is compared with the traditional ones by numerical simulations and measurements. Both the radiation and absorption characteristics are modeled. Modeling techniques, such as finite element and boundary element method and finite difference time domain methods, are applied and compared with each other.

5pAAb5. Evaluation of spatial information from artificial head and four-microphone array measurements. Joerg Becker and Markus Sapp (Insitut für Elektrische Nachrichtentechnik, RWTH Aachen, 52056 Aachen, Germany)

Examinations on the acoustic qualities of enclosures are mostly restricted to the evaluation of single-number quantities like "reverberation time" and "gravity time", or criteria which qualify the perceptual qualities, for example, "definition" or "clarity index." Most of these criteria are measured monaurally, although the spatial distribution of the reflections is essential for the acoustic quality of rooms. Spatial information can be registered with multimicrophone arrays or artificial heads. On the one hand it is easier to get the spatial information by using microphone arrays and on the other hand aurally adequate recordings can only be made with artifical heads. This paper focuses on the further development of algo- rithms for detecting virtual sound sources and compares evaluations of artifical head recordings with those made by a 4-microphone array.

5pAAb6. Combined beam tracing and Biot transfer-matrix model for predicting sound fields in enclosed spaces. Murray Hodgson, Andrew Wareing, and Callum Campbell (UBC Occupational Hygiene Prog. and Dept. of Mech. Eng., 3rd Fl., 2206 East Mall, Vancouver, BC V6T 1Z3, Canada, hodgson@mech.ubc.ca)

Numerous methods exist for predicting sound fields in enclosed spaces such as rooms, aircraft cabins, and the sea. These are generally energetic approaches which ignore phase and associated wave effects, and which describe sound reflection from surfaces by the energy absorption coefficient. Many, such as ray tracing, require long calculation times. In the work reported here, a new model is presented which is computationally efficient, includes phase, and decribes surface reflection by the angularly varying surface impedance. It is a triangular-beam-tracing algorithm, combined with a Biot-theory transfer-matrix model for laterally homogeneous, multilayer, poroelastic surfaces. The Biot model has been validated in the case of plates, porous materials, and seabeds. The combined approach is being validated in comparison with predictions by analytic and numerical approaches, and with experiment. The model is being used to study the effect of surface extended reaction on enclosed sound fields.

5pAAb7. Broadening the frequency range of panel absorbers by adding an inner layer of microperforated-plate. Jian Kang, Xueqin Zha, and Helmut V. Fuchs (Fraunhofer-Institut fur Bauphysik (IBP), Nobelstr. 12, D-70569 Stuttgart, Germany, kang@ibp.fhg.de)

A disadvantage of panel absorbers, namely a panel backed by an airspace, is that the effective frequency range is usually rather narrow. It has been theoretically demonstrated that the absorption range can be broadened by introducing a microperforated-plate between the panel and the back wall [J. Kang, IBP Report 1993]. In this paper, a series of experimental results on the effectiveness of this method is presented. The experiments were carried out using the single microphone FFT technology in an impedance tube. It is shown that with an inserted microperforated-plate the acoustic resistance of the whole structure is greater than that of the outer panel alone. When the parameters of the microperforated-plate are carefully selected, the absorption range can be effectively extended in both low- and high-frequency directions. Typically the improvement is around 1 oct. The effectiveness of the microperforated layer is much less when its resonance frequency is considerably higher than that of the outer panel alone. [Work supported by AvH.]

5pAAb8. Reverberation time predictions using neural network analysis. Joseph Nannariello and Fergus Fricke (Architectural and Design Sci. Dept., Univ. of Sydney, NSW 2006, Australia)

The study aims to utilize neural network analysis to develop a method to predict the reverberation time for enclosures-in the low and mid frequencies-by using neural networks that have been trained with constructional and acoustical data. The study begins with the hypothesis that, in practice, reverberation time predictions are too difficult to undertake using existing computer models, and too inaccurate when using other methods. Specifically, the study aims at providing an expeditious and accurate method of predicting the reverberation time of enclosures at the initial design stage. To substantiate the hypothesis, and to bring into effect the aims of the study, assessments are made of the predictive powers of the trained neural networks. The results of the investigations have indicated that there is a good basis for using trained neural networks to predict the reverberation time for enclosures. The results have also shown that neural network analysis can identify those variables that have an effect on the predicted reverberation times. 
5pAAb9. Sound fields caused by diffuse-type reflectors with periodic profile. Daiji Takahashi (Grad. School of Eng., Kyoto Univ., Kyoto, 606-01 Japan, tkhs@archi.kyoto-u.ac.jp)

For the purpose of providing highly diffuse reflections, the reflection surface is often periodically corrugated. The degree of diffusion of this periodic type diffuser can be evaluated by an objective criterion named DNSD [D. Takahashi, J. Acoust. Soc. Jpn. (E) 16, 51-58 (1995)], the value of which varies in the range $0-1 ; D N S D=0$ for a perfect diffusion, and $D N S D=1$ for a specular reflection. Optimum diffuse reflectors with periodic profiles can be designed by using this criterion, but this type of diffusion might have some aspect of misgivings due to its periodicity, which may be a kind of so-called "tone coloration." In other words, the highly diffuse reflections might have a problem of some particular effect on the spectrum caused by the interference between the direct and the reflected waves, and the effect might be different from an ordinary case of the plane surface. In this report, for the three types of reflectors, the calculated results of the SPL characteristics of the reflected field as well as the diffusion performances are presented and mutually discussed in comparison with the measured data from $\frac{1}{8}$-scale model experiments.

5pAAb10. Directivity patterns of small explosions. Miguel Arana (Phys. Dept., Public Univ. of Navarra, 31006, Pamplona, Spain)

There are methods and equipment for the generation, amplification, and emission of acoustic impulses in order to evaluate the acoustic characteristics of a room by means of the Fourier transform from its impulse response. However, the responses of this equipment (especially the inertia of the loudspeakers and its lack of linearity) produce acoustic signals that do not reproduce reliably the necessary acoustic impulses. A notable dispersion was found in the measurements accomplished with these systems. The directivity patterns of small explosions have been measured and analyzed with the purpose of using them as impulsive acoustic sources. The typical durations of these impulses are between 0.1 and $0.2 \mathrm{~ms}$. The sound power spectrum is relatively smooth from 1 to $10 \mathrm{kHz}$. The mean acoustic power is around $200 \mathrm{~W}$ (143 dB, Ref. $1 \mathrm{pW})$. For this an experimental device with 16 channels and software based on LabView were used. This communication will show the experimental device as well as the obtained results (mainly the dispersion of both the acoustic power and directivity patterns). [Work supported by Education Department of Government of Navarra.]

5pAAb11. Sound absorption measurements in diffuse field: A study of parameters. Marco Nabuco, Paulo Massarani (Acoust. Testing Lab., DIAVI/INMETRO), and Roberto Tenenbaum (Acoust. and Vib. Lab., COPPE/ Federal Univ. of Rio de Janeiro)

Many researchers have been studying the sound absorption coefficient measurement technique in reverberation chambers, since Sabine presented his formulation during the end of the last century, in order to find reasons for data frequently obtained bigger than $100 \%$, normally correlated to the edge effects of the sample. Some researchers have studied the implications of the lack of diffuseness on the sound absorption data. This paper presents the data obtained for the sound absorption coefficient measured in a 200- $\mathrm{m}^{3}$ reverberation chamber at the National Institute of Metrology, Standardisation and Industrial Quality-INMETRO, in Brazil, for different areas and configurations materials, from 1 to the standardized $12-\mathrm{m}^{2}$ sample. Also presented is the steady-state standard deviation, the crosscorrelation between microphones, and the shape of the decays curves to qualify the diffuseness both in steady as well as during the transient state. At last is presented the data obtained using a steady-state measurement of the sound absorption using a standard reference sound source calibrated in semianechoic chamber.
5pAAb12. Dependence of accuracy of reverberation time measurement on input filters. Dragana S. Sumarac (Faculty of Elec. Eng., P.O. Box 35-54, 11001 Belgrade, Yugoslavia, esumarad@ubbg.etf.bg.ac.yu)

In this paper, attention was paid to the standard method for measuring frequency characteristics of reverberation time in rooms. The influence of transfer function characteristics of $1 / 1$ and $1 / 3$ digital octave input filters on differences in measuring values of reverberation time was studied. Procedures for complete digital analysis of recorded impulse response of the room were designed. All procedures were designed by the MATLAB software package. The verification of that measuring software was performed on a great number of recorded impulses in real rooms. The several classes of $1 / 1$ and $1 / 3$ digital octave FIR and IIR filters were designed according to the international standard IEC 225 . The complete analysis was performed using the recorded reverberation decay curve in 20 rooms significantly different in volume and reverberation time values. The rooms were excited with broadband pink noise and short duration impulses. The values of measured reverberation times were statistically analyzed to show the expected variance that can appear due to differences of applied 1/1 and $1 / 3$ digital input filters.

5pAAb13. Reverberation time directly obtained from a squared impulse response envelope. Fumiaki Satoh (Dept. of Architecture, Chiba Inst. of Technol., Tsudanuma 2-17-1, Narashino-shi, Chiba, 275 Japan, f_satoh@cc.it-chiba.ac.jp), Yoshito Hidaka (Dept. of Elec. Eng., Tohwa Univ., Chikushigaoka 1-1-1, Minami-ku, Fukuoka-shi, 815 Japan), and Hideki Tachibana (Tokyo Univ., Tokyo, 106 Japan)

For the measurement of reverberation time, a method of directly regressing the envelope of a squared impulse response (direct method) was examined using 100 data obtained in 14 auditoria. The results were compared with those obtained by the integrated impulse response method (Schroeder method) and a very high coincidence was found between them. It has also found that the direct method is more robust to background noise than the Schroeder method and in the former method relatively short-time data will do compared with the latter method.

5pAAb14. Effects of flow resistance on acoustic performance of permeable elastic-plate absorbers. Manabu Tanaka (Bldg. Res. Corp., Osaka-Suita, 5650873 Japan) and Daiji Takahashi (Kyoto Univ., Kyoto, 6068317 Japan)

In general vibration theories of thin elastic plate, the plates have been assumed to be nonpermeable. However, previous work on permeable membranes revealed that the acoustic properties of a membrane are strongly affected by its permeability [Takahashi et al., J. Acoust. Soc. Am. 99, 3003-3009 (1996)]. In this study, permeable elastic-plate absorbers are proposed and their acoustic performance is examined by means of numerical analysis. For the simplest model, a theory for sound transmission through and sound reflection by a single permeable plate absorber with the oblique incidence of steady-state plane wave is developed. In this theory, permeability of the plate is represented by unit-depth flow resistivity [MKS-rayl/m]. Subsequently, for more complex models, structured absorbers composed of the facing of a permeable plate and several layers of air and/or absorptive materials are investigated theoretically. With these theoretical models, parametric surveys are executed to evaluate the acoustic performance of permeable elastic-plate absorbers. As a result of numerical calculations, it becomes clear that the flow resistance of the permeable elastic plate has a remarkable influence on its acoustic characteristics, especially at high frequencies. 
5pAAb15. Qualitative evaluations of the acoustics of rooms: Real room studies and headphone studies. Martin A. Gold (Architectural Acoust. Res. Group, 231 ARCH, Univ. of Florida, P.O. Box 115702, Gainesville, FL 32611-5702)

This pilot study attempts to qualify the use of headphones to evaluate the listening conditions of existing and proposed architectural spaces. Qualitative listening evaluations of speech and music were conducted in multiple locations in three different listening environments: a 2000-seat multipurpose concert hall, a 600-seat lecture room, and a 120-seat lecture room. Groups of college age students were asked to evaluate loudness, clarity, reverberance, spatial impression, and background noise using a questionnaire with a seven-point bipolar rating scale [Cervone (1990)]. The speech and music sources were played through two adjacent loudspeakers at the front of the respective rooms while the students completed the evaluation forms. The speech and music sources were binaurally recorded on a DAT at the listener locations in the respective rooms. At a later time, students were asked to evaluate the acoustical qualities of the binaural recordings made in the rooms using the same questionnaire. The recordings were presented to the listeners in a random order in a blind subjective evaluation. Data from real room evaluations and headphone studies are compared and conclusions are drawn regarding the potential for headphone listening experiments as a means to study qualitative aspects of room acoustics.

5pAAb16. On the acoustical characteristic of a balloon. Anthony P. Nash (Charles M. Salter Associates, 130 Sutter, Ste. 500, San Francisco, CA 94104, tnash@cmsalter.com)

It is common to use a transient acoustical signal when testing the properties of large architectural spaces. Some of the traditional signal sources include pistol shots, pulses from loudspeakers, etc. Assuming the background noise is reasonably low, an inflated balloon can be a very effective signal source in this application. The published literature contains little documentation regarding the sound power, directional properties, and repeatability of an exploding balloon. A carefully controlled experiment was conducted involving a series of balloons exploded in an environment where the closest reflecting surface was located more than 15 $\mathrm{m}$ from the source. Several microphones ringing the source were used to measure the free-field acoustical transient on a multi-channel tape recorder. The measured data will demonstrate that a properly inflated balloon is a very satisfactory (and lightweight!) sound source.

5pAAb17. Sound absorption by a large-size isolated acoustic resonator with a cylindrical cavity. V. J. Stauskis (Vilnius Gediminas Tech. Univ., Sauletekio al. 11, 2040 LT, Vilnius, Lithuania)

An acoustical structure consisting of a large-scale isolated resonator with a large-diameter cylindrical cavity may be used. This resonator differs essentially from the classical Helmholtz resonator whose cavity is only several millimeters in diameter and lined with a sound-absorbing material. Formulas are presented with which the impedance of the cavity, the impedances both inside and outside the cavity, and the impedance of the volume of the resonator are calculated. A radiation directivity pattern expressed by Bessel's function is used to find the radiation impedance. Calculations show that the sound energy is absorbed by resonators made of sound-reflecting materials. The larger the cavity diameter is, the larger the sound absorption. Absorption is of a resonant character with the resonant frequency at $60 \mathrm{~Hz}$. A resonator measuring $200 \times 200 \mathrm{~cm}$, with the cavity diameter $50 \mathrm{~cm}$ and the distance to the rigid surface being $30 \mathrm{~cm}$, absorbs $3.5 \mathrm{~m}^{2}$ of sound energy at the resonant frequency. At very low frequencies, changes in the imaginary parts of both cavity and radiation impedances occur along with the increase in the cavity diameter and frequency.
5pAAb18. Influence of distance and sound recording system on intelligibility and depth localization in highly reverberant conditions. Ruiz Robert (Laboratoire d'acoustique, Univ. de Toulouse-le Mirail, 5 allees Antonio Machado, 31058 Toulouse Cedex 1, France) and Ballet Isabelle (Univ. de Toulouse-le Mirail, 31058 Toulouse Cedex 1, France)

Speech intelligibility degrades when the listener moves from the source. In a highly reverberant and empty church, scores are measured at different distances from a loudspeaker and compared with those obtained from recordings of the speech at the same location. The phonetic material is a set of phonetically balanced lists of triphonemic French words. Two monophonic (omnidirectional, cardiod) and one stereophonic (ORTF) systems are used. Listening of recorded lists is performed with the same loudspeaker(s) as in the church. Results of the experiment indicate smaller variations of scores with the distance. An important improvement of scores is obtained with the stereophonic system. A second experiment consists of using a sound material composed of two voices performing a singing exercise edited in monophony in order to create two distinct sound planes. Listening tests allow the quantiication of the depth localization variations with distance and sound recording system. Results show a good respect of distance and reveal a sudden change in depth localization for stereophony. Acoustical properties of the church, distance factors of the microphones, properties of the stereophony against monophony, and real listening conditions are considered to discuss the experiments.

5pAAb19. Sound field in long enclosures with diffusely reflecting boundaries. Judicaël Picaut, Laurent Simon (LAUM UMR 6613, Univ. du Maine, 72017 Le Mans Cedex 9, France), and Jean-Dominique Polack (Univ. Paris VI, Case 161, 75252 Paris Cedex 05, France)

The sound field modelization by a diffusion equation is used to predict the sound propagation in long enclosures with diffusely reflecting boundaries. The diffusion equation is solved for time-varying sources and particularly in the steady state. Analytical expressions of sound attenuation and reverberation in infinite enclosures are given. Their simplicity makes them easy to apply to realistic diffusing long enclosures. For wide cross sections of enclosures and when absorption at the beginning and at the end walls is high, solutions for finite enclosures are also proposed. The source location and the absorption of each wall are taken into account in the model. Comparisons between analytical solutions, numerical simulations, and classical theory of diffuse sound field show good agreements. [Work supported by the PIR-Villes CNRS.]

5pAAb20. Reverberance of an existing hall in relation to subsequent reverberation time and SPL. Shigeo Hase (Grad. School of Sci. and Technol., Kobe Univ., Rokkodai, Nada, Kobe, 657-8501 Japan), Akio Takatsu (Showa sekkei, Inc., 1-2-1-8000, Benten, Minato-ku, Osaka, Japan), Shin-ichi Sato, Hiroyuki Sakai, and Yoichi Ando (Kobe Univ., Rokkodai, Nada, Kobe, 657-8501 Japan)

Psychological tests in relation to reverberance of sound fields were conducted in an existing hall which is a medium-sized multipurpose hall with 400 seats. Music and speech as sound sources were radiated from an omnidirectional dodecahedron loudspeaker on the stage. Pairedcomparison tests were applied for the sound fields changing two orthogonal factors. These factors were the subsequent reverberation time $T_{\text {sub }}$ and the SPL. The $T_{\text {sub }}$ were adjusted by a hybrid system involving the reverberation control room and an electroacoustic system. 21 subjects took part in this test. The results indicate that the reverberance is influenced independently on the $T_{\text {sub }}$ and the SPL. The reverberance increases with increasing both the $T_{\text {sub }}$ and the SPL. 


\title{
Session 5pAO
}

\section{Acoustical Oceanography and Animal Bioacoustics: Acoustics of Fisheries and Plankton V}

\author{
Manell E. Zakharia, Chair \\ CPE Lyon, LISA (EP 92 CNRS) LASSO, 43 Bd. du 11 Novembre 1918, BP 2077, 69616 Villeurbanne Cedex, France
}

\section{Invited Paper}

1:00

5pAO1. Bioacoustic resonance absorption spectroscopy. Orest Diachok (Naval Res. Lab., Washington, DC 20375)

\begin{abstract}
Absorption losses at the resonance frequencies of sardines up to about $18 \mathrm{~dB}$ at $1.3 \mathrm{kHz}$ at night, $15 \mathrm{~dB}$ at $1.7 \mathrm{kHz}$ during the day and $35 \mathrm{~dB}$ at $2.7 \mathrm{kHz}$ at dawn were observed at a range of $12 \mathrm{~km}$ during Modal Lion, a multidisciplinary experiment designed to isolate absorptivity due to fish from other effects on long-range propagation at a relatively shallow $(83 \mathrm{~m})$ site in the Gulf of Lion. Comparison of transmission loss measurements with a numerical sound propagation model that incorporates absorption layers in the water column permitted estimation of the average absorption coefficient, depth, and thickness of absorption layers. The depths and thickness of layers estimated from sound propagation measurements were in good agreement with echo sounder data. The measured resonance frequencies of dispersed fish at night were within $13 \%$ of theoretical computations, based on swim bladder dimensions which were derived from near coincident samples of adult $(\sim 16 \mathrm{~cm}$ long) sardines. A smaller absorption line at $3.9 \mathrm{kHz}$ at night is consistent with the resonance frequency of dispersed juvenile sardines, which are known to be $\sim 6 \mathrm{~cm}$ long, based on historical data. Measured resonance frequencies of sardines in schools were about 0.59 times the resonance frequency of dispersed sardines. The observed frequency shift is analogous to previously observed frequency shifts associated with "clouds" of bubbles. The results presented here suggest the possibility of long-term tomographic mapping of fish parameters over large areas using transmission loss measurements. [This work was supported by The Office of Naval Research.]
\end{abstract}

\section{Contributed Papers}

1:20

5pAO2. The application of wideband signals in fisheries and plankton acoustics. John E. Ehrenberg and Thomas C. Torkelson (Hydroacoustic Technol., Inc., 715 NE Northlake Way, Seattle, WA 98105-6429)

Over the last 30 years, there have been considerable improvements in techniques developed for acoustic assessment of fisheries and plankton populations. Techniques have evolved from analog echo integrators used to obtain crude abundance estimates to digital multiple-beam techniques for measuring in situ target strength, location, and velocity of individual targets. These advancements have been largely due to digital signal processing techniques applied to signals at the output of an echo sounder. Constant-frequency sine wave $(\mathrm{CW})$ pulses have been used with nearly all plankton and fisheries assessment systems. The same digital signal processing technology used to improve post-processing is now finding its way into biological assessment echo sounders that use FM slide/chirp signals. Systems with time-bandwidth products of up to 50 have been developed and implemented to provide a signal-to-noise advantage in excess of 15 $\mathrm{dB}$ when compared to $\mathrm{CW}$ pulse signals with the same spatial resolution. This paper describes the implementation and application of FM slide/chirp signals for echo integration and target strength measurement. Practical implementation issues, such as the use of pulse shaping for range side-lobe control, are discussed. Recent data collected with a multifrequency acoustic assessment system developed for WHOI are presented.

\section{$1: 35$}

5pAO3. Wideband fisheries sounder. From individual echoes analysis to classification of schools at sea. Manell E. Zakharia (CPE Lyon, LISA (EP92 CNRS)/LASSSO, 43 Bd du 11 Novembre 1918, BP 2077 69616 Villeurbanne Cedex, France, zakharia@cpe.fr)

Several works have been carried out in the last decade concerning wideband fisheries systems and their ability to discriminate fish species. The paper will first review previous work showing the relevance of wideband systems for fisheries acoustics (analysis of individual echoes). A short description of a wideband prototype (20 to $80 \mathrm{kHz}$ ) will then be given showing the technological issues. An emphasis will be made on the analysis of fish schools either in a controlled situation (lakes) or at sea. Wideband echo processing was based on autoregressive modeling of individual pings (modeling of spectrum resonances). This modeling concentrates the information contained in the wideband echo in a reduced set of parameters that could be used for classification. A neural network was trained to recognize several fish species. Ground truth was achieved by species identification, at sea, using associated trawling. For the construction of an echo data base, only monospecific schools were considered. Classification results at sea using only the spectral signature of the echoes were as high as $75 \%$ from a single ping. Lake tests have shown that the use of several pings (ten and above) could increase the recognition rate to more than $90 \%$.

\section{1:50}

5pA04. Searching for species identifiers in multifrequency target strength variability. James Dawson (BioSonics, Inc., 4027 Leary Way NW, Seattle, WA 98107)

Acoustic research has shown that the species of some schooling fish can be assigned based solely on measurement parameters from acoustic returns. For single targets, this process is complicated by the high variability of in-situ target strength (TS) measurements. Attempts at speciation based on the patterns of TS variability were confounded by interactions between acoustic frequency and fish directivity. Initial attempts at defining speciation parameters based on multifrequency target strength measures look promising. This paper reports on the results of simultaneously measuring the in-situ target strength of fish at 38-, 120-, and 420-kHz acoustic frequencies, and proposes the definition of several speciation parameters. 
5pA05. A broadband acoustic fish identification system. Gerald Denny and Patrick Simpson (Sci. Fishery Systems, Inc., P.O. Box 242065, Anchorage, AK 99524, scifish@alaska.net)

A broadband fish identification system has been built and tested in the Great Lakes, Prince William Sound, and the Bering Sea. Unique signatures have been obtained on free swimming and captive animals. These signatures include jellyfish, euphausiids, Pacific Rockfish, and Walleye Pollock. Acoustic characteristics and utilization of the system will be discussed, as well as results of in situ data collection.

\section{2:20-2:35 Break}

\section{2:35}

5pA06. The use of two freqencies to interpret acoustic scattering layers. Denise R. McKelvey (Natl. Marine Fisheries Service, NOAA, 7600 Sand Point Way NE, Seattle, WA 98115, denise.mckelvey@afsc.noaa.gov)

The use of multiple frequencies during acoustic stock assessment surveys allow methods to be used, which can discriminate between different species groups based on their scattering properties and assist in target species identification, thus improving estimates of biomass. Research, using multiple frequencies to study zooplankton, have used the difference in backscatter between several frequencies to identify the species or species groups comprising the scattering layers. This paper presents the results of using a dual-frequency differencing algorithm on 120 - and $38-\mathrm{kHz}$ mean volume backscatter strength (MVBS) data collected during a 1995 United States west coast acoustic survey of Pacific hake (Merluccius productus) as a means to acoustically separate hake from zooplankton. Scatterers were sampled using various nets with mouth areas ranging from 5 to 1000 $\mathrm{m}^{2}$. Backscatter data from both frequencies were corrected for background noise. The effect of species composition, time of day, depth, and density of scatterers on the (delta)MVBS data were evaluated. Results indicate that the dual-frequency differencing algorithm shows a marked difference between fish and zooplankton (delta)MVBS and can be used to distinguish between fish and zooplankton scattering layers.

\section{2:50}

5pA07. Fish'n krill: A 38/120-kHz acoustic separation. Yvan Simard (Dept. of Fisheries and Oceans, Maurice-Lamontagne Inst., P.O. Box 1000, Mont-Joli, QC G5H 3Z4, Canada, simardy@dfo-mpo.gc.ca)

Volume backscattering data (Sv) at 38 and $120 \mathrm{kHz}$ were collected in the Gulf of St. Lawrence, during daylight, in regions where krill scattering layers (SL) and pelagic fish (mainly capelin) were present. The SLs and fish aggregations were sampled with the Bioness zooplankton samples and with small-mesh pelagic trawls equipped with acoustic monitoring systems. Echointegration was performed on small bins of $\sim 5 \mathrm{~m}$ vertically and $\sim 50 \mathrm{~m}$ horizontally. Comparisons of $\mathrm{Sv}$ at the two frequencies indicated that krill SLs had stronger backscattering at 120 than at $38 \mathrm{kHz}$, as expected. The ratio was relatively constant within the same SL, but varied from one SL to the other in parallel with the size of krill. The backscattering from fish schools or fish aggregations or fish layers was stronger at 38 $\mathrm{kHz}$ than at $120 \mathrm{kHz}$. This permitted the separation of fish from krill on the echograms, even for complex cases where fish were embedded in krill SL or had structural patterns that mimic the signature of krill SL. The 38/120-kHz backscattering ratio appears therefore useful to separate krill from fish, or vice-versa, for better interpretation and estimation of these taxa individually.
5pAO8. Multifrequency acoustic assessment of fisheries and plankton resources. Thomas C. Torkelson (Hydroacoustic Technol., Inc., $715 \mathrm{NE}$ Northlake Way, Seattle, WA 98115-6429), Thomas C. Austin, and Peter H. Wiebe (Woods Hole Oceanogr. Inst., Woods Hole, MA 02543)

The range of target species encountered in marine biological resource assessment and the inability to obtain a broad view of these resources by direct physical sampling techniques has lead to the utilization of various indirect measurement techniques. Among these techniques is the use of acoustics (sonar) to determine biomass distribution through integration and target strength measurements. WHOI is using a multifrequency acoustic system to obtain more accurate measurements of target species on the Georges Bank. The system uses advanced acoustic techniques to enhance detectability at the lower trophic levels and uses an advanced towing technique to enable sampling over the water column to depths in excess of 200 $\mathrm{m}$. This presentation discusses the challenges encountered with indirect acoustic sampling and the techniques implemented to meet them. The system to be discussed operates at frequencies between $38 \mathrm{kHz}$ and 2 $\mathrm{MHz}$, using either FM slide (chirp) or traditional CW acoustic pulses.

\section{$3: 20$}

5pA09. Pulse compression processing of zooplankton echoes. Joseph D. Warren (MIT/WHOI Joint Prog. in Oceanogr. and Ocean Eng., Woods Hole Oceanogr. Inst., Woods Hole, MA 02543, jwarren@whoi.edu), Timothy K. Stanton, Dezhang Chu (Woods Hole Oceanogr. Inst., Woods Hole, MA 02543), and Duncan E. McGehee (Tracor Appl. Sci., San Diego, CA 92123-4333)

A pulse compression process was used to analyze echoes from individual animals of three distinct morphological groups of zooplankton. The groups studied were fluidlike animals (Euphausiids, decapod shrimp), elastic-shelled (Gastropods), and animals containing a gas inclusion (Siphonophores). A broadband chirp signal was used to insonify freshly collected animals that were then tethered in a seawater tank either in the laboratory or on the deck of a ship. The decapod shrimp was tethered to a stepper motor and was rotated in 1-deg increments. The other experiments had some animals fixed and others moving freely. The pulse compression processing of the echoes from the animals temporally resolved multiple returns from an individual. Using existing scattering models [Stanton et al., J. Acoust. Soc. Am. (to be published)], the temporal and amplitude differences of the returns were used to estimate size and orientation. Estimates of the size of the animal were made for the Euphausiids, Gastropods, and Siphonophores, while orientation estimates were made for the decapod shrimp. These results agreed well with the actual dimensions of the animals.

\section{$3: 35$}

5pAO10. Multistatic, multifrequency scattering from zooplankton. C. F. Greenlaw, D. V. Holliday, and D. E. McGehee (Tracor Appl. Sci., Inc., 4669 Murphy Canyon Rd., San Diego, CA 92123)

Very satisfactory results have been obtained in estimating size abundances of small zooplankton such as copepods from inversion of multifrequency backscattering measurements. Application of this method has become almost routine in many situations. Fluctuations in the scatterer physical properties (density and compressibility) tend principally to cause errors in the abundance estimates whereas size estimates are largely unaffected. The model commonly employed for small crustacean scatterers, a truncated-mode version of the Anderson fluid sphere model, predicts bistatic scattering as well as monostatic backscattering. The behavior of bistatic scattering at angles approaching 90 deg predicted by this model shows that scattering spectra at these angles are quite sensitive to physical properties. This suggests that simultaneous measurements at multiple frequencies and one or more off-axis angles might permit estimation of the physical properties of zooplanktonic scatterers as well as their sizes. Preliminary modeling has been done for a single fluid scatterer to illustrate 
the potential for simultaneous estimation of size, density, and compressibility of zooplanktonic organisms in a laboratory setting. The character of the bistatic scattering may argue for nontraditional methods for inverting this sort of data.

\section{3:50}

5pA011. Laboratory studies and theoretical modeling of bistatic scattering of fish. Li Ding (VITech Innovative Res. and Consulting, Victoria, BC V8P 3MB, Canada) and Zhen Ye (Natl. Central Univ., Taiwan, R.O.C.)

Bistatic scattering of fish, in particular forward scattering (scattering in the forward direction), has been identified as a potential means of detecting fish, supplementary to the traditional backscattering. A series of laboratory experiments has been conducted over the past 2 years to measure bistatic scattering characteristics of fish, including forward scattering strength and scattering patterns around the forward direction. It is observed that the forward scattering strength increases rapidly with acoustic frequency and is much stronger than the backscattering strength. The measured scattering patterns (at 120 and $200 \mathrm{kHz}$ ) suggest that the scattering is strongest in the forward direction when the incident wave is normal to the fish, and drops by $10-20 \mathrm{~dB}$ when the scattering angle deviates from the forward direction by a few degrees. In the latter case, the scattering strength is still much stronger than backscattering strength. These results are compared with a recently developed deformed cylinder model, and with a shelled cylinder model currently under development. [Work supported by the Science and Technology Agency of Japan, the Department of Fisheries and Oceans of Canada, and the National Central University of Taiwan.]

FRIDAY AFTERNOON, 26 JUNE 1998

EAST BALLROOM B (S), 1:30 TO 4:50 P.M.

\title{
Session 5pBV
}

\section{Bioresponse to Vibration/Biomedical Ultrasound and Physical Acoustics: Lithotripsy II}

\author{
Robin O. Cleveland, Cochair \\ Department of Aerospace and Mechanical Engineering, Boston University, 110 Cummington Street, \\ Boston, Massachusetts 02215 \\ Andrew J. Coleman, Cochair \\ Department of Medical Physics, St. Thomas Hospital, Lambeth Palace Road, London SE1 7EH, England
}

\section{Invited Papers}

$1: 30$

5pBV1. Shock wave measuring techniques in liquids. Wolfgang Eisenmenger (Physikalisches Inst., Univ. of Stuttgart, Pfaffenwaldring 57, 70550 Stuttgart, es@pi1.physik.uni-stuttgart.de)

The parameters of shock waves in liquids as used for lithotripsy have been measured with needle-, membrane-, and fiber optic probe hydrophones. The true rise times of planar shock waves are determined by surface detection or by the transient response of piezoelectric crystals. The different measuring techniques are compared with respect to their suitability for the determination of shock wave parameters, such as risetime, pulse width, positive and negative pressure amplitudes, and also with respect to aging, calibration sensitivity lifetime, etc. The fiber optic probe hydrophone appears as an optimum high precision standard for calibrated shock wave measurements in lithotripsy. Finally, aspects of the shock parameters with respect to the stone destruction efficiency are discussed.

\section{1:50}

5pBV2. Full-wave modeling of lithotripter fields. Eckard Steiger (Inst. für Hoechstfrequenztechnik und Elektronik/Akustik, Univ. of Karlsruhe, D-76128 Karlsruhe, Germany, eckard.steiger@etec.uni-karlsruhe.de)

The propagation of extracorporeal shock wave lithotripter pressure pulses is considered. A computational modeling must account for the effects of diffraction, nonlinear steepening and generation, and propagation of shock waves. Some commercial lithotripters are reflector focusing systems. Therefore, reflections at curved boundaries have to be considered. For specific interests in the propagation through and the interaction with human tissue structures, refraction, absorption, and scattering must be included. A full-wave model based on a set of nonlinear acoustic time domain equations is applied. This system is discretized to obtain a finite difference representation of broad bandwidth and low dispersive character. To enable an accurate shock wave computation an algorithm that modifies the temporal and spatial resolution adaptively is developed. Exemplary a reflector focusing lithotripter is modeled. To validate the model resulting computed wave profiles are compared with measured ones. The measurements are performed using a fiber-optic probe hydrophone. Excellent agreements show the method to provide an accurate and flexible tool for field predictions of complex devices. Characterizing parameters like $-6-\mathrm{dB}$ focal regions and energy quantities can be obtained, too. A human tissue model is applied to obtain predictions of the relevant parameters inside the human body. 
5pBV3. Acoustic shock-wave induced cavitation: A comparison of theory and experiment. Andrew J. Coleman and Mark D. Cahill (Medical Phys. Directorate, Guys' \& St. Thomas' Hospital Trust, Lambeth Palace Rd., London SE1 7EH, UK, a.coleman@umds.ac.uk)

A calibrated membrane hydrophone has been used to make measurements of pressure in a cavitating field generated in water by a single $30-\mu$ s duration ultrasound pulse with a $0.2-\mathrm{MHz}$ center frequency and a peak negative pressure $\left(p_{-}\right)$variable from 0.5 to 5 $\mathrm{MPa}$ as employed in clinical lithotripsy. The acoustic emission from cavitation in the vicinity of the hydrophone is identified as the high-frequency ( $>1 \mathrm{MHz}$ ) component of the waveform. This "cavitation component" is compared with theoretical predictions of the pressure radiated by a bubble made using the Gilmore model [C. C. Church, J. Acoust. Soc. Am. 86, 215-227 (1989)]. The model was run for initial bubble radii of $1-100 \mu \mathrm{m}$ using the measured pressure waveform to drive the radial dynamics of the bubble. The theory predicts a transition from completely driven dynamics at $p_{-}$below $3 \mathrm{MPa}$ to largely undriven dynamics above $3 \mathrm{MPa}$. This transition, which is substantially independent of bubble size, is experimentally observed and occurs at a measured $p_{-}$of $3 \pm 0.4 \mathrm{MPa}$. The time between the pressure pulse and the first undriven inertial collapse is predicted to increase approximately twice as rapidly with increasing amplitude as is measured. A mechanism resulting in shorter than predicted collapse times is discussed. [Work supported by the Medical Research Council, UK.]

5pBV4. A new shock pressure waveform to amplify transient cavitation effect. Dominique Cathignol (INSERM U281, 151 Cours Albert Thomas, 69424 Lyon Cedex 03, France, cathignol@lyon151.inserm.fr), Jahangir Tavakkoli, Françgoise Chavrier, Alain Birer, Alex Arefiev, and Mathieu Prigent

Shock-wave-induced tissue damage and/or stone disintegration are greatly dependent on cavitation effect. In order to control this effect in tissue, a new piezocomposite shock-wave generator $(200 \mathrm{~mm}$ in diameter and focused at $190 \mathrm{~mm}$ ) has been developed with the capability of producing two different kinds of shock pressure-time waveform at its focus (type 1: a tensile wave followed by a compressive wave, and type 2: a compressive wave followed by a tensile wave). The cavitation effects produced by these two pressure waveforms were compared in the following experiments: (1) measurement of cavitation bubble collapse time (defined as the time difference between the shock wave arrival and the main bubble collapse), (2) study of cavitation-induced destructive effects in the agar gel, (3) study of disintegration rate on plaster balls (a kidney stone-mimicking material), and (4) study of cavitation-induced destructive effects on rabbit liver in vivo. All of these experiments revealed the amplified effect of transient cavitation produced by the type 1 pressure-time waveform. Comparisons with a computed simulation using the Gilmore Akulichef model, taking into account the rectified diffusion, confirm the experimental results. It is concluded that the temporal characteristics of the shock pressure waveform have a major influence on transient cavitation.

\section{2:50}

5pBV5. Stone tracking with time-reversal techniques. J. L. Thomas and M. Fink (Laboratoire Ondes et Acoustique, Ecole Superieure de Physique et de Chimie, Industrielles de la Ville de Paris, Universite Denis Diderot, 10 rue Vauquelin, 75231 Paris Cedex 05, France)

Time reversal of ultrasonic fields allows a very efficient approach to focusing pulsed ultrasonic waves through lossless inhomogeneous media. Time reversal mirrors (TRM) are made of large transducer arrays, allowing the incident acoustic field to be sampled, time reversed and reemitted. Time reversal processing permits a choice of any temporal window to be time reversed, allowing operation in an iterative mode. In multitarget media, this process converges on the most reflective target, i.e., the dominant scatterer. The time reversal process is applied to track, in real time a moving gall bladder or kidney stone embedded in its surrounding medium. The feasibility of adaptative beam forming techniques to track the stone during a lithotripsy treatment is investigated. It is shown that TRM allows sharp focusing on one bright point of the stone. Once the bright point is selected, a time of flight profile is determined and used in a least-mean-square method to calculate the spatial coordinates of the stone. Stone trajectories can be tracked by this technique at $30 \mathrm{~Hz}$.

\section{3:10-3:25 Break}

\section{3:25}

5pBV6. On a mechanism of target disintegration at a shock wave focusing in ESWL systems. Valery K. Kedrinskii (Lavrentyev Inst. of Hydrodynamics, Lavrentyev prospect 15, Novosibirsk 630090, Russia)

It is well known how the lithotriptors operate and the discussion concerning the mechanisms of target disintegration are continued. Meanwhile, to understand these mechanisms means to obtain a possibility to influence on the fracture process. The spall mechanism, as a result of acting with the shear forces, the crack growth inside a stone, and an erosion mechanism (impacts of cumulative microjets forming at bubble collapse in the cavitation zone near a target wall), were suggested by Sturtevant, Kuwahara, Delius, Takayama, Groenig, Crum, and others to explain the disintegration effects observed. At the same time, all authors do not deny a rise of the cavitation zone in the vicinity of a focus under the action of a negative pressure phase in "a tail" of a shock wave. However, the role of the cavitation cluster is interpreted in different ways assuming that this effect is a secondary one. This paper proposes an approach to the estimation of the disintegration mechanism, which is based on the development and dynamics of the cavity cluster in a focus vicinity. Within the framework of a two-phase model of cavitating liquid the problem of bubbly cluster development and its collapse on a solid wall are considered. The experimental data on a simulation of cavity cluster pulsation on a target and the idea that hydraulic impacts generated by cluster can determine the mechanics of target disintegration in a focusing zone of shock wave and its rarefaction phase are discussed. 
5pBV7. First in vitro experiments using a new reflector to concentrate shock waves for extracorporeal shock wave lithotripsy. Achim M. Loske and Fernando E. Prieto (Inst. Física, UNAM, México D.F., México)

Electrohydraulic lithotripters to perform extracorporeal shock wave lithotripsy (ESWL) treatments use symmetrical reflectors in order to concentrate the energy generated in one of their foci $(F 1)$. The new reflector presented here was designed in an attempt to increase the efficiency of ESWL. It is obtained by combining sectors of two rotationally symmetric ellipsoidal reflectors with different separations between their foci $F 1$ and $F 2$. These sectors are joined together in such a way that the $F 1$ foci coincide, creating a separation between the $F 2$ foci. The purpose is to temporally and spatially dephase the shock wave generated at $F 1$. This is achieved because the initial shock wave is divided, by reflection, into two shock fronts converging toward two, rather than one, $F 2$ foci. Pressure measurements, and in vitro experiments using kidney-stone models and dog kidneys, indicate that the new design could be more efficient in breaking up renal stones, without producing more tissue damage. The performance of the new reflector was compared with one having the geometry of a Dornier HM3 (nonmodified) reflector. Both reflectors were installed separately in an electrohydraulic experimental shock wave generator (MEXILIT II). [Work supported by DGAPA IN502694, UNAM.]

\section{Contributed Papers}

\section{4:05}

5pBV8. Use of two pulses to control cavitation in lithotripsy. Michael R. Bailey (Appl. Phys. Lab, 1013 NE 40th St., Seattle, WA 98105), Robin O. Cleveland (Boston Univ., Boston, MA 02215), David T. Blackstock (Appl. Res. Labs., Austin, TX 78713), and Lawrence A. Crum (Appl. Phys. Lab., Seattle, WA 98105)

In lithotripsy acoustic cavitation appears to play a role in both comminution of kidney stones and damage to tissue. To investigate the role of cavitation, we used a pair of confocal electrohydraulic lithotripters (ellipsoidal reflectors). A delay in firing of $\Delta t$ modified the cavitation field without altering the peak positive and negative pressures. Bubble collapse time $t_{c}$, measured by passively detecting acoustic emissions generated by the bubble, was used as a measure of cavitation activity. A two-pulse sequence from the source shortened $t_{c}$ relative to the single pulse value $t_{c 1}$. Collapse times calculated by using the Gilmore equation agreed well with measurements. Relative intensity of bubble collapse was determined by depth of pits created in aluminium foil. For pulse delays $\Delta t<0.3 t_{c 1}$ the pit size decreased, a result that implies the second pulse stifled the collapse. Conversely, for delays in the range $0.5 t_{c 1}<\Delta t<t_{c 1}$, cavitation apparently intensified, since pit depth increased by a factor of up to 2 . Pit depth correlates well with (calculated) pressure radiated by the bubble. When the two reflectors were pointed at each other and fired simultaneously, pitting was localized to a spot less than $1 \mathrm{~cm}$ in diameter. [Work supported by ONR, ARL:UT IR \& D, and NIH PO1-DK43881.]

\section{4:20}

5pBV9. Detection and control of lithotripsy-induced cavitation in blood. Brenda Jordan (Dept. of Biol., Tennessee State Univ., Nashville, TN 37209-1561), Michael R. Bailey (Appl. Phys. Lab., Seattle, WA 98105), Robin O. Cleveland (Boston Univ., Boston, MA 02215), and Lawrence A. Crum (Appl. Phys. Lab., Seattle, WA 98105)

Monitoring and controlling cavitation offer ways of isolating the role of cavitation in lithotripsy-induced cell damage. Finger cots containing 7 $\mathrm{ml}$ of PBS-diluted blood (3\% hematocrit) were treated by 100 lithotripter pulses focused by either a conventional rigid reflector or a pressure-release reflector. The rigid reflector produced $7 \%$ hemolysis at $18 \mathrm{kV}$. The pressure-release reflector, which produces an equally strong acoustic pulse but less intense cavitation, produced $1 \%$ hemolysis which was indistinguishable from control levels. A passive cavitation detector consisting of two confocal focused transducers operating in coincidence was used to monitor cavitation within each blood sample. With the rigid reflector, each transducer recorded a spike when pre-existing bubbles were collapsed by the positive portion of the lithotripter pulse and a second spike when the bubbles, caused to grow by the negative portion of the pulse, underwent inertial collapse. The time between spikes was 1.25-1.5 times longer in blood than in water. The results appear to indicate that transient cavitation is an important mechanism in lithotripsy-induced hemolysis, that lithotripter-induced cavitation can be monitored in cell samples, and that bubble dynamics in blood and water are not identical. [Work supported by NIH PO1 DK43881, UW STAR, and DARPA/ONR.]

\section{$4: 35$}

5pBV10. Experiments on the relation of shock wave parameters to stone disintegration. Thomas Dreyer, Rainer E. Riedlinger, and Eckard Steiger (Inst. für Hoechstfrequenztechnik und Elektronik/Akustik, Univ. of Karlsruhe, Kaiserstr. 12, D-76128 Karlsruhe, Germany, thomas.dreyer@etec.uni-karlsruhe.de)

The sound field of different focusing piezoelectric transducers designed for lithotripsy was investigated. The shock wave parameters according to proposed standards, e.g., FDA Draft 1991, were determined. The parameters achieved are based on measurements using a fiber-optical probe hydrophone. In contrast to PVDF-based hydrophones, this hydrophone is able to give a correct representation also of the tensile components of a complete signal and provides a higher spatial resolution. Thus some shock wave parameters like beam energy can be calculated more precisely. The different lithotripter pulses were applied to model stones in vitro, recording the amounts of removed material. Fragmentation results were compared with the different parameters. Attempts were made to arrange these parameters according to their relevance to disintegration. The evaluation of these experiments revealed up to now that there is no significant relation between some of the shock wave parameters and fragmentation efficiency. It is concluded that most of the proposed parameters do not describe well the efficacy of different lithotripter sources on concrements. 


\title{
Session 5pEAa
}

\section{Engineering Acoustics: Scattering and Radiation}

\author{
Sung-Hwan Ko, Chair \\ Naval Undersea Warfare Center, Code 2133, Newport, Rhode Island 02841
}

\section{Contributed Papers}

\section{1:00}

5pEAa1. Scattering by a horizontal strip on a hard side wall. Djamel Ouis and Sven Lindblad (Eng. Acoust., LTH, Lund Univ., P.O. Box 118, S-221 00, Lund, Sweden, djamel.ouis@kstr.lth.se)

The study of the effect of a horizontal hard striplike element on a side wall is presented. This may give an estimate of the usefulness of such elements in reinforcing the early sound components in a listening room. The theoretical calculations are based on geometrical assumptions in combination with a diffraction model to take into account the diffraction by the edge of the scattering element. This latter is based on the time-domain theory of Biot and Tolstoy for the diffraction by a hard wedge applied to the half-plane. An accurate and useful approximation for the diffracted field in the frequency domain using Fresnel integrals is presented. Some measurements on a scale model are also presented.

\section{1:15}

5pEAa2. Acoustic wave scattering from a coated cylindrical shell. Sung H. Ko (Naval Undersea Warfare Ctr. Div., Newport, RI 02841)

A theoretical model was developed to evaluate the effect of a coating on a cylindrical shell. The coating is designed to reduce the flexural wave noise generated by the vibration of the cylindrical shell. In underwater applications, this coating is called an inner decoupler. The outer surface of the composite structure, which consists of the cylindrical shell and the inner decoupler, is in contact with water; the core (cavity) of the structure contains air. The analysis of the problem is made for a double-layer cylindrical structure that is immersed in water. The theory of elasticity, the elastic wave propagation, the acoustic wave propagation, and pertinent boundary conditions are used in formulating the problem. The twodimensional problem is treated in this study. This implies that all the quantities are independent of the axial coordinate. Numerically calculated results for both coated and uncoated cylindrical shells are presented to compare these two directivity patterns.

\section{1:30}

5pEAa3. Numerical solution of the linearized Euler equations with high-order centered schemes. John A. Ekaterinaris (Nielsen Eng. and Res., 526 Clyde Ave., Mountain View, CA 94043-2212)

The unsteady, linearized Euler equations governing propagation of acoustic disturbances in the presence of a uniform free stream or sound scattering from solid surfaces are solved numerically. The governing equations are discretized in space using the standard fourth- and sixth-order central-difference schemes, and compact finite-difference schemes with spectrallike resolution are also used. The semidiscrete system of equations obtained after space discretization is marched in time using high-order, Runge-Kutta schemes. Numerical boundary conditions are applied at the far field in order to limit the extent of the computational domain. Solutions are computed first on Cartesian, equally spaced grids. Computed results for propagation of a pulse in a uniform medium are compared with exist- ing analytic solutions. Next, sound scattering from a cylinder surface is computed using a nonuniform body-fitted numerical mesh. The effects of grid spacing, time accuracy, the method used for space discretization, and the boundary conditions on the computed solutions are investigated.

\section{$1: 45$}

5pEAa4. Modeling acoustic radiation from end-initiated explosive line charges. William J. Marshall, Jr. (BBN Technologies, Union Station, New London, CT 06320, bmarshall@bbn.com)

Acoustic radiation produced by end-fired explosive line charges has been examined experimentally and theoretically. In terms of directivity effects, line charges are found to behave like beam-steered continuous arrays over moderately wide bands. This talk briefly summarizes measured waveforms and derives a math model which predicts levels and spectrum shapes from such sources over a wide range of view angles and source characteristics. The model is driven by two parameters which may be extracted from experimental data. The result is a practical means of designing explosive line arrays to desired source levels, bandwidths, and beam patterns. [Work supported by DARPA.]

2:00

5pEAa5. Resonant frequencies in degeneration of hybrid longitudinal and torsional vibration system. LiQun Zhang and TieYing Zhou (Dept. of Modern Appl. Phys., TsingHua Univ., Beijing, PROC)

The Langevin vibrator is made of the longitudinal and torsional piezoelectric plates, which can be used for an ultrasonic motor and an ultrasonic welding transducer. Because of the difference between the longitudinal and torsional sound velocity, their resonant frequencies are not equal. To make resonant frequencies of two vibration modes in degeneration, a matching block with a thin neck was connected to the end of the vibrator. Two calculation methods, a dimension network which transmits matrixes and a finite element method have been used. The feasibility of frequencies tuning through a matching block has been analyzed and how to effect the resonant frequency by the radius and thickness of the thin neck and matching block has been summarized. The resonant frequencies of several steel cylinders with variant thin necks and matching blocks were calculated. Some experimental results of measuring the resonant frequencies have been compared with the calculation. According to the calculation and experimental results, a prototype of hybrid longitudinal-torsional ultrasonic motor has been manufactured, and it can be operated very well [Tomikawa et al., IEEE Trans. Ultrason. Ferroelectr. Freq. Control 39 (1992)]. 
5pEAa6. The estimation of an interior sound field by source methods. Gee-Pinn James Too and Shing Maw Wang (Dept. of Naval Architecture and Marine Eng., Natl. Cheng Kung Univ., 1 Ta-Hsueh Rd., Tainan, Taiwan 70101, R.O.C.)

In an earlier study, three different source methods (SSM, similar source method; IPSM, internal parallel source method; ISM, internal source method) were successfully developed to estimate radiation and fields which are not suitable for estimating interior sound fields. In this study, the similar source method is modified to estimate the interior sound field. The modification is to move the sources to somewhere outside the boundary surface in order to estimate the interior sound field and to prevent the singularity problem when the source point is close to the field point. Finally, the sound field inside a 2D infinite cylinder and in a car will be estimated. The results will be compared with the results obtained by the boundary element method.

FRIDAY AFTERNOON, 26 JUNE 1998

EAST BALLROOM A (S), 3:00 TO 4:30 P.M.

\title{
Session 5pEAb
}

\section{Engineering Acoustics: Acoustic Waveguides}

\author{
Anthony A. Atchley, Chair \\ Graduate Program in Acoustics, Pennsylvania State University, P.O. Box 30, Applied Science Building, \\ State College, Pennsylvania 16804
}

Contributed Papers

3:00

5pEAb1. Insertion loss measurements of an acoustical enclosure by using Intensity and MLS methods. Pedro Cobo, Carlos Ranz, Salvador Santiago, Jose Pons, Manuel Siguero, and Carmen Delgado (Instituto de Acustica, CSIC, Serrano 144, 28006 Madrid, Spain, iacpc24@fresno.csic.es)

The Insertion Loss (IL) is one of the most appropriate descriptors for the acoustical performance of an enclosure. The IL is conventionally measured by comparing either sound power of space-averaged sound-pressure measurements before and after enclosing the sound source. In this paper, a comparison is made between a power-based IL measurement, using a sound intensity meter, and a pressure-based IL measurement, using the MLS method. Special attention is paid to practical and technical aspects of both methods, such as time consuming, low-frequency limit, signal-tonoise ratio, and background noise immunity. [Work supported by CICYT, Project AMB97-1175-C03-01.]

\section{3:15}

5pEAb2. Intensity measurements in various rooms: A new intensity probe. W. F. Druyvesteyn (Philips Res. Labs., Eindhoven, The Netherlands) and H. E. de Bree (MESA Res. Inst., The Netherlands)

The main advantage of acoustic intensity measurements is that in a reverberant room the direct and the reverberant sound field can be determined. This property has been tested using the Bruel \& Kjaer $p$ - $p$ probe in four different rooms (anechoic, reverberant and two listening rooms). As compared to the results in the anechoic room, small deviations, which can be positive and negative, were found. The deviations can be explained using a model of early reflections. A new intensity probe consisting of a microphone and a sound velocity sensor has been tested and compared with the Bruel \& Kjaer $p$ - $p$ probe. The sound (particle) velocity sensor contains the Microflown, a novel micro-machined sensor. This siliconbased velocity microphone operates on a thermal principle. The dimensions of the Microflown are on the order of a cubic millimeter. For symmetry considerations, however, the sensors were packaged in a quarter inch package. Experiments were done in the anechoic and in the reverberation room. A good agreement was found.
3:30

5pEAb3. In situ absorption measurements using a transfer function technique and MLS. G. Dutilleux, U. R. Kristiansen, and T. E. Vigran (Norwegian Univ. of Sci. and Technol., NTNU, Acoust. Group, Dept. of Telecommunications, N-7034 Trondheim, Norway, tor.erik.virgran@tele.ntnu.no)

The transfer function (or two-microphone) technique is a tool for measuring materials absorption related parameters as impedance or absorption coefficients. But this method is laboratory bound in its original form due to free field assumption and practical issues. It is shown that after proper modifications the transfer function technique can be used successfully in noisy and reverberating fields and work well at low frequency even in small measurement rooms, becoming therefore a really in situ measurement technique. A wide frequency range implementation is presented, based on a two-channel MLS measurement system, "energy ratio invariant" time windowing, a basic specular reflection propagation model with spherical decoupling, and a particular geometrical configuration. The measurement method is tested on a wide range of absorbers. For impedance measurements using infinite surface propagation models the uniqueness of the inversion procedure is investigated using the conformal mapping technique. In addition, faster inversion and model computation routines are proposed. Measurement results obtained under realistic in situ conditions are provided and compared with reference methods.

\section{$3: 45$}

5pEAb4. Lumped impedance of a planar discontinuity in an acoustic waveguide. Ralph T. Muehleisen (Dept. of Civil, Environ., and Architect. Eng., Univ. of Colorado, Boulder, CO 80309) and Anthony A. Atchley (Penn State Univ., P.O. Box 30, State College, PA 16804)

When modeling planar discontinuities in acoustic waveguides at low frequencies, the standard assumptions involve continuity of pressure and volume velocity at the discontinuity. While continuity of volume velocity is required by mass conservation, excitation of evanescent higher-order modes and thermoviscous loss suggest that pressure is not continuous across the discontinuity. Such a pressure loss can be modeled by a series acoustic impedance. A new model for a lumped series impedance at a planar waveguide discontinuity has been developed and is presented. The model uses a modal expansion of the acoustic field near the discontinuity and matrix techniques to obtain a closed-form approximation for the re- 
sistive and reactive elements of the series impedance. As an example of the accuracy of the model, the prediction of the resonance frequency of a constricted annular resonator is presented. [This work was supported by the Office of Naval Research and the American Society of Engineering Education.]

\section{4:00}

5pEAb5. A short water-filled pulse tube for the measurement of the acoustic properties of materials at low frequencies. Debra M. Kenney (Carderock Div., Naval Surface Warfare Ctr., 9500 MacArthur Blvd., West Bethesda, MD 20817, kennde@oasys.dt.navy.mil) and Peter H. Rogers (Georgia Inst. of Technol., Atlanta, GA 30332)

A method of measuring the acoustic properties of materials at low frequencies in a water-filled pulse tube, which was a fraction of a wavelength long, was investigated. The method employed active sound cancellation at the end of the tube opposite from the sample, and signal processing of two hydrophone signals to separate the signal incident on the sample from its reflection. Two analytical models were developed and an experimental tube was built to examine the feasibility of such measurements. The results from the simulation and experiment suggest that the short water-filled pulse tube would be useful for measuring compliant materials at moderately low frequencies (down to $300 \mathrm{~Hz}$ ), but not for measuring high impedance materials or for making measurements at very low frequencies. The simulation and experiment showed that the thicker the sample, relative to a wavelength, the lower the sensitivity to experimental errors. Fortunately, many of the materials of interest in underwater acoustics are compliant materials with relatively slow phase velocities and consequently shorter wavelengths. The impedance of the backing behind the sample also influences the sensitivity of results to errors in the measurements. [Work supported by ONR and NSF.]

\section{$4: 15$}

5pEAb6. The generation of low-frequency acoustic waves in electrolytes. Bronislaw Zoltogorski (Wroclaw Univ. of Technol., Inst. of Telecommunication and Acoust., Wybrzeze Wyspianskiego 27, 50-370 Wroclaw, Poland)

The phenomenon of the generation of acoustic waves in liquids based on interaction of alternating ion current and constant magnetic field is analyzed. The properties of such a magneto-electric transducer having no mechanical vibrating elements is examined theoretically (with the use of an electric equivalent circuit) and experimentally. The following measurements were carried out: (1) amplitude of conductivity current as a function of frequency, (2) amplitude of acoustic pressure at the distance of $0.5 \mathrm{~m}$ without constant magnetic field and (3) with magnetic field. For water from a municipal water-pipe network, the upper limit frequency of the current characteristic is 4 to $5 \mathrm{kHz}$. The same frequency properties exhibit a pressure characteristic. The directional pattern of the source depends on the shape of the electrodes and can be optimized for special aims. The energetic efficiency of the transducer is not great because the majority of the electric power is converted into heat and causes an increase in temperature of the liquid. That process is relative to a big value of resistance of weak electrolytes. That type of transducer can be applied in liquids without ion conductivity by submerging a container with very thin rubber walls and with mounted electrodes and filling with an electrolyte.

FRIDAY AFTERNOON, 26 JUNE 1998

ASPEN ROOM (S), 12:55 TO 6:00 P.M.

\title{
Session 5pPAa
}

\section{Physical Acoustics: Sonochemistry and Sonoluminescence: SL II}

\author{
R. Glynn Holt, Cochair \\ Department of Mechanical Engineering, Boston University, 110 Cummington Street, Boston, Massachusetts 02215 \\ Thomas J. Matula, Cochair \\ Applied Physics Laboratory, University of Washington, 1013 NE 40th Street, Seattle, Washington 98105
}

Chair's Introduction-12:55

\section{Invited Papers}

\section{1:00}

5pPAa1. Energy focusing in bubbly flows. Seth Putterman, Keith Weninger, Robert A. Hiller, a) and Bradley P. Barber ${ }^{\text {b) }}$ (Phys. Dept., Univ. of California, Los Angeles, CA 90095)

Sonoluminescence, cavitation damage at surfaces, and cavitation in accelerating flows are realizations of spectacular levels of energy focusing in nature. In a resonant sound field a single trapped bubble of gas can focus the ambient sound energy by 12 orders of magnitude to generate a clocklike string of picosecond flashes of ultraviolet light. [Barber et al., "Defining the unknowns of sonoluminescence," Phys. Rep. 281, 65 (1977)]. In more complicated geometries a high level of sound leads to the formation of hemispherical bubbles attached to an exposed surface. These bubbles also emit light and in addition damage the surface. Measurements show that the pulsation of these bubbles maintains the hemispherical symmetry [Weninger et al., "Sonoluminescence from an isolated bubble on a solid surface," Phys. Rev. E 56, 6745 (1997)], thus raising the question as to whether cavitation damage is due to (micro)jets or imploding (hemispherical) shock waves. Finally, flow through a Venturi tube generates a stream of bubbles which also emit subnanosecond flashes of light [F. B. Peterson and T. P. Anderson, Phys. Fluids 10, 874 (1967)]. Luminescence from an isolated trapped bubble in water seems to work well with any noble gas, whereas luminescence from cavitating flows and surface bubbles is quite dependent on xenon [argon bubbles appear to give no light at all]. The width of the SL flash [Gompf et al., Phys. Rev. Lett. 79, 1405 (1997), Hiller et al., Phys. Rev. Lett. 80, 1090 (1998)] has been found to be independent of wavelength suggesting that 
light is emitted from a new high energy phase of matter-probably a cold dense nano-plasma. The key unknowns of SL are the size and temperature of the hot spot from which the light is emitted. Experiments aimed at measuring these quantities will be discussed.

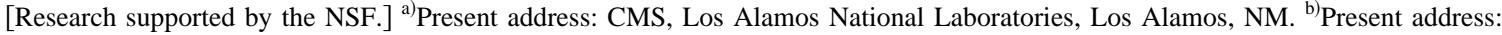
Lucent Technologies, Murray Hill, NJ.

$1: 20$

5pPAa2. Sonoluminescence stability for gas saturations down to 0.01 Torr. Robert E. Apfel and Jeffrey A. Ketterling (Dept. of Mech. Eng., Yale Univ., 9 Hillhouse Ave., New Haven, CT 06520-8286)

Water solutions with very small argon partial pressures were investigated. The experiments were performed in a sealed cell. The argon was used in both a pure form and in mixtures with other gases. A regime of stable sonoluminescence was seen at lower driving pressures for argon partial pressures down to 0.01 Torr. Mie scattering, light pulse measurements, and visual observations over tens of thousands of cycles were used to determine if the bubble was stable. As the drive pressure was increased, the bubble moved into a slow time scale instability on the order of several seconds which sometimes caused break up. These experiments are discussed and compared to other published data involving gas mixtures at saturation levels up to $1 \mathrm{~atm}$.

\section{1:40}

5pPAa3. Evolution of a sonoluminescence bubble under a magnetic field. J. B. Young, H. Cho, and W. Kang (James Franck Inst. and Dept. of Phys., Univ. of Chicago, 5640 S. Ellis Ave., Chicago, IL 60637)

Studies of sonoluminescence (SL) in magnetic fields up to $20 \mathrm{~T}$ have revealed a striking magnetic field dependence. The intensity of emitted light is suppressed under increasing magnetic fields and vanishes above threshold magnetic field that depends on the applied sound pressure. Further increase in the magnetic field leads to the destruction of the bubble through dissolution. At a constant magnetic field, the light intensity is found to increase roughly linearly with increasing sound pressure until the bubble disappears above a cut-off pressure which depends on the strength of the magnetic field. The cut-off pressure is found to approximately double between 0 and $20 \mathrm{~T}$. Further study of the SL bubble through the measurement of the phase of the emitted radiation relative to acoustic drive shows a large increase in phase under magnetic fields. These results suggest that the bubble grows significantly in size and that there may be a substantial modification of bubble dynamics under magnetic field. The origin of the observed effects, possibly due to magnetic field-induced anisotropies in the SL bubble, will be discussed in light of our recent anisotropy studies under magnetic fields.

\section{2:00}

5pPAa4. Computed spectral and temporal emissions from a sonoluminescing bubble. William C. Moss and David A. Young (Lawrence Livermore Natl. Lab., L-200, 7000 East Ave., Livermore, CA 94551, wmoss@1lnl.gov)

Although the mechanism of emission in single bubble sonoluminescence (SBSL) has still not been identified conclusively, the “plasma" model [Moss et al., Science 276, 1398-1401 (1997)] appears to explain more features of SBSL than other models. Recent measurements of the light pulses emitted by SBSL [Gompf et al., Phys. Rev. Lett. 79, 1405-1408 (1997)] show that the pulse widths may be longer than the original UCLA measurements and can vary between 60 and $250 \mathrm{ps}$. These new results provide information for improving the modeling of the energy loss and emission mechanisms in the plasma model. In particular, the plasma can be described more accurately as a "strongly coupled plasma" (a plasma with a small Debye screening length). The main difference between the strongly coupled plasma and our earlier plasma model is that there is less energy loss by electron thermal conduction in the strongly coupled plasma, which gives rise to longer calculated pulse widths that are consistent with the new experimental data. [This work was performed under the auspices of the U. S. Department of Energy by Lawrence Livermore National Laboratory under Contract No. W-7405-Eng-48.]

5pPAa5. Gas dynamics of mixtures within sonoluminescence bubbles. Andrew Szeri and Brian Storey (Dept. of Mech. Eng., Univ. of California-Berkeley, Berkeley, CA 94720-1740)

Sonoluminescence experiments are often conducted using mixtures of gases intentionally dissolved in the liquid, and, presumably, resident in the bubble interior. Even when only a single gas is dissolved in the host liquid, vapor is undoubtedly present within the bubble in addition to the intended gas. Hence, all sonoluminescence experiments can be assumed to involve gas mixtures even though the contents of the bubbles cannot be directly assayed. Two constituents of a gas mixture will have differing solubility in the host liquid, which complicates the amount of each species one would expect is resident within a bubble, depending on how the experiment was prepared. Moreover, there is little opportunity for mixing of the bubble contents due to the highly spherically symmetric nature of single-bubble sonoluminescence. Using the methods of computational fluid dynamics, the fate of gas mixtures within a strongly driven bubble is investigated. [Work was supported by a grant from the National Science Foundation.]

\section{2:40-3:00 Break}


5pPAa6. Time-resolved measurements of optical emission from sonoluminescence. D. Froula, R. W. Lee, W. C. Moss, P. E. Young (Lawrence Livermore Natl. Lab.), and T. J. Matula (Univ. of Washington, Seattle, WA 98105)

The results of detailed measurements of sonoluminescence from a gas bubble that is acoustically driven in deionized water are presented. The pulse width and relative jitter of the optical flash and the minimum and ambient radius of the bubble as a function of the driving pressure, temperature, and gas concentration of the water are measured. The optical flash is collected by an f/1.4 lens, and then optically relayed onto the slit of a Hamamatsu C1587 optical streak camera which records the time evolution of the flash with picosecond resolution. Single event streaks are obtained, showing FWHM pulse widths of 200-400 ps, comparable to recently reported results [Gompf et al., Phys. Rev. Lett. 79, 1405 (1997)]. The jitter of the flash between events has been found to be a minimum of $\pm 200 \mathrm{ps}$. Mie scattering has been used to measure the maximum and ambient bubble radius at the same time for comparison to the model [Moss et al., Science 276, 1398 (1997)]. The consequences of these results on modeling will also be discussed. [This work was performed under the auspices of the U.S. Department of Energy by the Lawrence Livermore National Laboratory under Contract No. W-7405-Eng-48.]

3:15

5pPAa7. Single-bubble sonoluminescence: Acoustic emission measurements with a fiber-optic probe hydrophone. Bruno Gompf, Zhaoqiu Wang, Rainer Pecha, Wolfgang Eisenmenger (1. Physikalisches Inst., Univ. of Stuttgart, Pfaffenwaldring 57, D-70550 Stuttgart, Germany), and Ralf Guenther (Natural and Medical Sci. Inst., Eberhardtstr. 29, D-72762 Reutlingen, Germany)

In single-bubble sonoluminescence additionally to the short light pulses, the bubble emits in the collapse phase a sound wave which can be measured with a fiber-optic probe hydrophone [Staudenraus and Eisenmenger, Ultrasonics 31, 267 (1993)]. This type of hydrophone is an absolute ultrasonic wideband reference standard. Compared to piezoelectric hydrophones it allows measurements with higher spatial $(0.1 \mathrm{~mm})$ and temporal $(10 \mathrm{~ns})$ resolution. The intensity and the width of the emitted sound wave increase with increasing driving pressure, but vary only slightly with water temperature in contrast to the emitted light intensity. The total radiated energy of the sound wave is below $10 \%$ of the initial energy of the bubble. The results are compared with earlier measurements on transient cavitation bubbles and with new theoretical results.

3:30

5pPAa8. Luminescence from spherically and aspherically collapsing laser-induced bubbles. Claus-Dieter Ohl, Olgert Lindau, and Werner Lauterborn (Drittes Physikalisches Institut, Univ. of Göttingen, Bürgerstr. 42-44, D-37073 Göttingen, Germany, ohl@physik3.gwdg.de)

Luminescence from laser-induced cavitation bubbles (SCBL) is investigated experimentally. This experimental technique offers several advantages over the acoustical driving technique for luminescence studies: The optical resolution of the imaging system allows the investigation of the shape of the luminescence spot. This technique facilitates the study of the spherical asymmetries during the bubble collapse phase. Finally, complications due to gas diffusion and Bjerknes forces are avoided. Comparison with theory gives good agreement between the size of the luminescence spot and the observed minimum bubble size for spherical symmetry. A rigid boundary greatly influences the light output; SCBL is only observed for a mildly aspherical bubble collapse.
5pPAa9. On the theory of supercompression of a gas bubble in a liquid-filled flask. Robert I. Nigmatulin, Iskander Sh. Akhatov, Nelya K. Vakhitova (Ufa (Bashkortostan) Branch of Russian Acad. of Sci., $6 \mathrm{~K}$. Marx St., Ufa, 450000, Russia, iskander@ncan.ufanet.ru), and Richard T. Lahey, Jr. (Rensselaer Polytechnic Inst., Troy, NY 12180-3590)

The spherically symmetric problem of the oscillations of a gas bubble in the center of a spherical flask filled with a compressible liquid that is excited by pressure oscillations on the flask wall is considered. A generalization of the Rayleigh-Plesset equation for a compressible liquid is given in the form of two ordinary difference-differential equations that take into account the pressure waves that are reflected from the bubble and those that are incident on the bubble from the flask wall. The initial value problem for the initiation of bubble oscillations due to flask wall excitation and for the evolution of these oscillations is considered. Linear and nonlinear periodic bubble oscillations are analyzed analytically. Nonlinear resonant and near-resonant solutions for the bubble nonharmonic oscillations, which are excited by harmonic pressure oscillations on the flask wall, are obtained. The influence of heat transfer phenomena on the bubble oscillations is analyzed.

\section{4:00}

5pPAa10. Observations of single-bubble sonoluminescence in simulated microgravity and hypergravity. Jeremy E. Young, Nathaniel K. Hicks, A. C. Binner, Susan Richardson, Mark J. Marr-Lyon, and Philip L. Marston (Dept. of Phys., Washington State Univ., Pullman, WA 99164-2814)

Single-bubble sonoluminescence (SBSL) in water occurs when a bubble undergoes highly nonlinear volume oscillations in a sound field. In the presence of gravity, the SBSL bubble experiences a buoyancy force described by $\rho g V(t)$. There is also a Bjerknes force from the sound field. The time average of these two forces balances at the equilibrium location. If the SBSL apparatus is in normal gravity the bubble is displaced from the pressure antinode of the acoustic standing wave [Matula et al., J. Acoust. Soc. Am. 102, 1522-1524 (1997)]. Because of this displacement, the bubble has a vertical oscillation. One method of exploring the effects of the translational oscillations is to compare the light output of SBSL in microgravity to the light output of SBSL in normal or hypergravity. These were examined by an experiment performed aboard NASA's KC-135A. The airplane performs parabolic maneuvers, giving short periods of microgravity and hypergravity (approximately 0 and $2 \mathrm{~g}$, respectively). The results suggest a correlation of higher light output in microgravity than in hypergravity. [Work supported by Flags Up Feed, Inc., Space Grant Consortium, NASA, and W.S.U.]

\section{4:15}

5pPAa11. Analytical results for the conditions of shock formation inside SBSL bubbles. Ralf Guenther (Natural and Medical Sci. Inst., Eberhardstr. 29, D-72762 Reutlingen, Germany)

The mechanism of light emission during single-bubble sonoluminescence is still an unresolved question. Of central importance in this respect is whether a converging shock forms inside the bubble. Using an expansion of the fields inside the bubble, a set of dimensionless equations is derived, which allows exact solutions as well as perturbative treatment during different stages of the bubble collapse. The transition from nonshock to shock solutions is obtained as a function of dimensionless con- 
stants characterizing the dynamics. The explicit form of spatial distributions of temperature, velocity, and density is derived and consequences for the light emission are discussed

\section{$4: 30$}

5pPAa12. Shock formation in a sonoluminescing gas bubble. Takeru Yano (Mech. Sci. Dept., Hokkaido Univ., Sapporo, 060 Japan, yano@lebes-me.eng.hokudai.ac.jp)

Whether a shock is formed or not in a sonoluminescing gas bubble is a fundamental problem in the sonoluminescence study. The problem is numerically studied by solving Euler equations of gasdynamics in conjunction with an approximate equation of Keller type for the motion of the bubble radius. A high-resolution TVD upwind finite-difference scheme is used for Euler equations and a fourth-order Runge-Kutta method for a Keller-type equation. The result shows that, owing to the strong nonlinearity of the bubble motion, a slight increase of the driving pressure over a critical value makes the maximum collapse speed of a bubble wall supersonic, and hence the gas behavior changes from a nearly incompressible and adiabatic flow to a compressible one including a shock wave. The relation between the critical driving pressure and other parameters (the driving frequency, the initial bubble radius, etc.) is analyzed. The maximum temperature and pressure attained in the bubble are compared with those in earlier theoretical and numerical works.

\section{$4: 45$}

5pPAa13. Thermal wave from a sonoluminescing gas bubble. YoonPyo Lee, Sarng-Woo Karng (Korea Inst. of Sci. and Technol., P.O. Box 131, Cheongryang, Seoul, Korea), and Ho-Young Kwak (Chung-Ang Univ., Seoul, 156-756, Korea)

Heat diffusion from the sonoluminescing gas bubble which produces a high temperature and pressure environment adjacent to the bubble wall was considered by solving the heat diffusion equation numerically. The boundary conditions employed in this calculation are the time-varying bubble wall temperature obtained from the analytical solutions around the collapse point. The thermal boundary layer was found to be decreased rapidly during the collapse as expected. The heat flux at the collapse exceed $3 \mathrm{GW} / \mathrm{m}^{2}$. After the collapse a solitonlike heat wave, which was generated due to the rapid increase in the bubble wall temperature at/near the collapse for the sonoluminescing gas bubble, was found to propagate through the medium. On the other hand, the liquid temperature near the bubble decreases after the collapse for the nonsonoluminescing case. [Work supported by Korea Science and Engineering Foundation.]
5pPAa14. Effect of quantum radiation pressure on the bubble dynamics in sonoluminescence. Weizhong Chen and Rongjue Wei (Inst. of Acoust. and State Key Lab. of Mod. Acoust., Nanjing Univ., Nanjing 210093, PROC, wzchen@nju.edu.cn)

In the theoretical frame of quantum vacuum radiation (QVR), the effect of the QVR on the gas bubble dynamics in the single bubble sonoluminescence is studied. The dominated hydrodynamic equation of the gas bubble trapped in a liquid, Rayleigh-Plesset equation, has been modified by considering the reaction of the radiation force on the bubble surface and therefore causing a loss of energy. In the modified model the collapse and expansion of the bubble, especially near the turnaround at minimum radius, related to photon emission instead of independence on it. The numerical computation shows that the emission energy due to the QVR seems too low to explain the experimental data, if some present values of parameters, such as the ambient radius of the bubble and the sound pressure in liquid, are used. The radiation energy due to the QVR, however, can arise by changing those parameters. Based on this, it is concluded that the QVR may be a possible candidate for mechanisms of the sonoluminescence. [Work supported by NSF of China.]

\section{$5: 15$}

5pPAa15. Sonoluminescence: The effect of magnetic fields in the Planck theory. T. V. Prevenslik (2E Greenery Court, Discovery Bay, Hong Kong)

Sonoluminescence (SL) observed in the cavitation of water may be explained by the Planck theory of SL that treats the bubbles as collapsing miniature masers having a Planck energy intrinsic to the dimensions of the maser cavity. Microwaves at frequencies proportional to the collapse velocity are created in the bubble wall molecules as the Planck energy at optical frequencies is absorbed. Because of the interaction between the microwaves and molecular dipole moments, the Planck energy accumulates in the electronic state of polar molecules with a rotation quantum state. As the bubble wall is polarized, intense electrical fields develop to charge the bubble surface molecules to visible photon levels. By this theory, SL can only be observed if the maser walls close to optical dimensions thereby allowing the bubble wall molecules to accumulate Planck energy to the 3-6-eV levels necessary to produce VIS-UV photons. Closure to optical dimensions is assured by imposing a net positive collision pressure. The consistency of the Planck theory of SL was assessed with data on the effects of applied magnetic fields on SL. The net pressure was taken as the difference between the stagnation pressure in bringing the liquid bubble walls to rest and the pressure generated by the magnetic field in the presence of a charged bubble wall. Consistency with the Planck theory of SL is found as the SL intensity with magnetic field is parabolic and the peak SL intensity is linear with collapse velocity. 


\title{
Session 5pPAb
}

\section{Physical Acoustics: Nonlinear Acoustics II: 1. Solitons; 2. Biomedical Nonlinearities}

\author{
Mack A. Breazeale, Cochair \\ National Center for Physical Acoustics, University of Mississippi, Coliseum Drive, University, Mississippi 38677 \\ Lev A. Ostrovsky, Cochair \\ NOAA/ERL/ETL, 325 Broadway, R/E/ET-1. Boulder, Colorado 80303
}

Chair's Introduction-1:00

Invited Papers

1:05

5pPAb1. Chaotic dynamics in acoustics. Werner H. Lauterborn (Drittes Physikalisches Inst., Univ. of Göttingen, D-37073 Göttingen, Germany)

The theory developed for the description of chaotic and nonlinear dynamical systems also pertains to acoustics. A survey is given of the new ideas and methods that for experimentalists rely on the embedding of experimentally obtained data (time series) into high-dimensional spaces. The embedding is the starting point of nonlinear time series analysis for determining the static (fractal dimensions) and dynamic (Lyapunov exponents) properties of the point sets (attractors) obtained. Acoustical systems that can be investigated with these methods are, for instance, vibrating liquid surfaces (Faraday experiment), vibrating machines, musical instruments, nonlinear oscillators, speech and hearing, bubble dynamics, and acoustic cavitation.

\section{$1: 25$}

5pPAb2. Nonlinearities in the bioeffects of ultrasound. Edwin L. Carstensen (Dept. of Elec. Eng. and Rochester Ctr. for Biomed. Ultrasound, Univ. of Rochester, Rochester, NY 14627)

Biological effects of ultrasound involve nonlinear phenomena (1) in the propagation of sound itself, (2) in the generation of acoustic cavitation, and (3) in the biochemistry, physiology, and pathology of the biological system. Most nonthermal bioeffects of interest to users of diagnostic ultrasound require acoustic pressures great enough that the wave can become distorted by nonlinear propagation. Under limiting conditions this process can increase the absorption parameter of weakly absorbing media by orders of magnitude and make the absorption parameter of a material such as water as great as the linear absorption coefficient of liver tissue. At low amplitudes the response of the bubbles is dominated by the acoustic pressure. At a critical acoustic pressure, however, the inertia of the surrounding medium becomes controlling. At this threshold of acoustic pressure a $10 \%$ or $20 \%$ increase in acoustic pressure leads to an increase in the collapse pressure in the bubble by orders of magnitude. The rates of biochemical processes, including denaturation of biological macromolecules, are exponential functions of the temperature. Whether the physical process of heating by ultrasound is linear or nonlinear, this leads to a very strong nonlinear dependence of thermal tissue damage and teratological effects upon the levels of ultrasound.

\section{1:45}

5pPAb3. Solitary waves and solitons in acoustics. Nobumasa Sugimoto (Dept. of Mech. Eng., Fac. of Eng. Sci., Univ. of Osaka, Toyonaka, Osaka, 560 Japan)

This study reports on the propagation of acoustic solitary waves and solitons in ordinary air. In theory, it can be predicted by using a long and rigid tube with an array of Helmholtz resonators (or more generally, compact and closed side branches) connected to it with equal axial spacing. In the framework of nonlinear acoustics, the theory exploits three assumptions: (1) a plane wave over the tube, (2) continuum approximation for the array, and (3) negligibly small dissipation. It can be shown that solitary waves are compressive, that their speed is subsonic but faster than a threshold value determined by the array, and that the solitary waves tend to solitons of Korteweg-de Vries type asymptotically as the speed approaches the threshold. Thus it may be possible to implement a "soliton tube." As the speed approaches the linear speed of sound, the height of the solitary waves becomes greater, approaching the limiting value. Discussions are given on amplification of solitary waves and also on solitary waves in a tube with a double array of resonators. 


\section{Contributed Papers}

5pPAb4. Some consideration of processes of soliton formation from an initially sinusoidal wave form in a thin fiber of fused silica. Akira Nakamura (Dept. of Elect. Eng., Fukui Inst. of Technol., 3-6-1, Gakuen, Fukui, 910 Japan)

Soliton formation processes in a thin fiber of fused silica by computer simulation are discussed. It has already been found that tone-burst sinusoidal sound successively makes solitons from negative half-periods coming in order in the wave train. In this case, the first soliton made from the first negative period is the most stable with respect to the following solitons, because some disturbances caused by preceding positive pressure periods are delayed and superposed with following negative pressure periods. Then, the pressure profiles of these subsequent negative periods are changed. Therefore, nonlinear distortion is affected. In this paper the intention is to discuss the difference of soliton formation processes between two single sinusoidal pulses with preceding negative and positive periods. A preceding negative period makes a stable soliton without any disturbance generated from the following positive pressure part, while the disturbance from the preceding positive period gives change to the pressure profile of following negative period, then nonlinear distortion of this negative period is affected. As a result, frequency spectra of both are differing with the propagation. Discussion is made by operational treatment using a nonlinear distortion operator and linear propagation operator.

\section{2:20}

5pPAb5. Fast spectral algorithm for modeling focused sound beams in a highly nonlinear regime. Vera A. Khokhlova (Dept. of Acoust., Phys. Faculty, Moscow State Univ., Moscow 119899, Russia, vera@na.phys.msu.su), Michalakis A. Averkiou (ATL Ultrasound, Bothell, WA 98041-3003), Steven J. Younghouse, Mark F. Hamilton (Univ. of Texas at Austin, Austin, TX 78712-1063), and Lawrence A. Crum (Univ. of Washington, Seattle, WA 98105)

Numerical modeling of high-intensity focused sound beams is important for medical applications of ultrasound. The KZK equation, which may be modified to include relaxation in the time domain or arbitrary absorption and dispersion in the frequency domain, is widely used to model ultrasound beams in tissue. Numerical solutions are available in either the time or frequency domain, but they become time consuming at high intensities when thin shocks are developed. To speed up calculations, a known high-frequency asymptotic result for shocks can be incorporated in the frequency domain algorithm. In comparison with exclusively numerical schemes, the present approach substantially reduces the number of harmonics and thus the computation time [Yu. A. Pishchal'nikov, O. A. Sapozhnikov, and V. A. Khokhlova, Acoust. Phys. 42, 362-367 (1996)]. Focused cw beams radiated from Gaussian and piston sources are simulated in water and biological tissue. The accuracy of the asymptotic approach is verified via conventional numerical methods of solution. It is shown that the present asymptotic method provides accurate results with a considerable reduction in computation time. In combination with conventional numerical schemes, the developed asymptotic method provides an effective tool for investigating focused sound beams for a wide range of parameters. [Work supported by NIH PO1-DK43881, Fogarty Research Program, and CRDF.]

\section{2:35}

5pPAb6. Acoustic nonlinearity calculations using the Tait equation of state. Bruce Hartmann, Gilbert F. Lee, and Edward Balizer (Naval Surface Warfare Ctr., West Bethesda, MD 20817-5700)

Beyer's $B / A$ parameter of acoustic nonlinearity was calculated using the Tait equation of state supplemented with specific heat results, in the same manner as done earlier with our equation of state [J. Acoust. Soc. Am. 82, 614 (1987)]. Calculations were made of sound speed, soundspeed temperature and pressure derivatives, the two components of $B / A$, and the value of $B / A$ for a series of six n-alkane liquids as well as the limiting case of molten polyethylene. All these results were compared with experimental data from the literature. In addition, a comparison of the results with Ballou's rule (linear relation of $B / A$ versus reciprocal sound speed) was made. Overall, values calculated using the Tait equation are found to agree with experiment about as well as our equation of state, though the pressure derivatives of the sound speed and the final value of $\mathrm{B} / \mathrm{A}$ are generally not as accurate using the Tait equation. [Work supported by the Naval Surface Warfare Center's In-house Laboratory Independent Research Program.]

5pPAb7. Acoustic streaming near Albunex spheres. Gerard Gormley and Junru Wu (Dept. of Phys., Univ. of Vermont, Burlington, VT 05405)

Acoustic streaming near bubbles in an external standing-wave sound field has been an interesting research topic for decades. It was relatively difficult to visualize the acoustic streaming pattern experimentally, especially for small bubbles due to the instability of the bubbles and difficulties associated with the optical technique. Using Albunex spheres (a contrast agent for ultrasonic imaging), a specially designed microscope device, and a video peak store technique, several acoustic streaming patterns around a single and a pair of Albunex spheres of tens of micrometers radius in a $160-\mathrm{kHz}$ ultrasonic standing wave field have been recorded. Spatial peak pressure amplitude of the standing wave was estimated to be in the order of $0.5 \mathrm{MPa}$ by using optical interferometry. The acoustic streaming velocity was in the range of $50-100 \mu / \mathrm{s}$

\section{3:05-3:20 Break}

\section{$3: 20$}

5pPAb8. Acoustical nonlinearity and its images in biomedicine. $X$. F. Gong, D. Zhang, S. G. Ye, X. Z. Liu, X. Chen, and W. Y. Zhang (Inst. of Acoust., State Key Lab. of Modern Acoust., Nanjing Univ., Nanjing, 210093, PROC)

Some acoustical nonlinear effects in biological media at medical ultrasonic frequencies and intensities were studied experimentally. These phenomena include wave distortion, harmonic generation, energy transformation, extra-attenuation, departure of intensity response curve from linear relation, etc. The influence of these effects on medical ultrasonic diagnosis and therapy was discussed. As a basic parameter in nonlinear acoustics, the acoustical nonlinearity parameter can describe the above-mentioned nonlinear effects and provide some information on the structure and composition of biological tissues. Thus the imaging method of an acoustical nonlinearity parameter using secondary wave detection was developed and investigated. Nonlinearity parameter tomography for normal and a series of pathological porcine tissues was presented. The results obtained in experiments indicate that the difference between normal and diseased tissue in nonlinearity parameter tomography is more sensitive than that in linear parameter tomography. Therefore, nonlinearity parameter tomography may become a novel imaging method for tissue characterization. [Work supported by NSF of China.]

\section{3:35}

5pPAb9. Determination of the acoustic nonlinearity parameter $B / A$ using a phase locked ultrasonic interferometer. Jevon R. Davies, Jonathan Tapson (Univ. of Cape Town, Elec. Eng., Private Bag, Rondebosch, 7700, South Africa), and Bruce J. P. Mortimer (Cape Technikon, Cape Town, 8000, South Africa)

The acoustic nonlinearity parameter $B / A$ is an important parameter for characterizing acoustic propagation in fluids. Recent developments in the fields of shock waves and biomedical ultrasonics have necessitated an accurate method for measuring this quantity. This paper presents a new technique based on an ultrasonic interferometer that allows accurate measurements of the change in sound speed within a liquid cavity. The system makes use of a novel continuous wave phase locking principle, which has 
the effect of nullifying many of the nonlinearities inherent in the acoustic interferometer. The phase lock circuitry automatically adjusts the frequency so as to keep an exact integer number of waves in the cavity regardless of changes in sound speed. Using this principle, $B / A$ is determined by relating a small change in ambient pressure (less than 2 bar) to a change in frequency under isentropic conditions. $B / A$ values are calculated using this experimental setup for several liquids including two slow sound-speed fluorocarbon liquids, FC-43 and FC-75. These liquids are unique in that they have the lowest range of sound speeds of any organic liquids and in that they have the highest values of $B / A$ recorded thus far.

\section{3:50}

5pPAb10. Experimental measurement and numerical modeling of nonlinear propagation in biological fluids. Victor F. Humphrey (Dept. of Phys., Univ. of Bath, Claverton Down, Bath BA2 7AY, UK), Prashant K. Verma (Leicester Royal Infirmary, Leicester LE1 5WW, UK), and Francis A. Duck (Royal United Hospital, Combe Park, Bath BA1 3NG, UK)

The nonlinear pressure fields generated in three biological fluids (amniotic fluid, urine, and a $4.5 \%$ human albumin solution) by a circular 2.25-MHz transducer have been investigated experimentally using a flexible measurement facility and compared with numerical predictions. The acoustic field was generated by a single element 38-mm-diameter transducer coupled to a PMMA lens with a focal gain of 12 in water for source pressures from 0.007 to $0.244 \mathrm{MPa}$. The experimental results illustrate significant harmonic generation in all three fluids which may have safety and imaging implications. In addition, the facility was used to make low level measurements of the acoustic parameters of the fluids, including the frequency dependence of attenuation, at both room and physiological temperature. This data has been used in conjunction with a finite difference model based on the Khokhlov-Zabolotskaya-Kuznetsov equation (the Bergen Code) to model the experiments. The agreement with experiment was found to be generally good, indicating that this type of model may be useful for predicting in-vivo pressure distributions given an accurate knowledge of the medium parameters. [Work supported by EPSRC].

\section{4:05}

5pPAb11. Asymptotically unified exact solutions of the Khokhlov-Zabolotskaya equation. Yuri N. Makov (Dept. of Phys., Moscow State Univ., Moscow 119899, Russia,

makov@acs364.phys.msu.su)

For the large distance traversed by the sonic beam the exact analytical and physically realistic solutions of the Khokhlov-Zabolotskaya (KZ) equation were found for the first time. The procedure of finding these solutions as the self-similar traveling waves identifies their asymptotical generality with respect to the initial arbitrary wave profiles. The resulting solutions consist of the periodically repeating parts of the second-order curvers such as hyperbolas, ellipses, and, in particular, parabolas. The boundaries between these parts are defined with the use of the preserved integrals of the KZ equation. The influence of nonlinearity, diffraction, which is seen in many-dimensional cases, and initial curvature of the phase front of the wave beam on the forming of the typical form of a specific asymptotical wave profile ( $\mathrm{N}$ shaped or $\mathrm{U}$ shaped) was discussed. A comparison was made between analytical solutions with the numerical results for the $\mathrm{KZ}$ equation and the experimental data and a full agreement was discovered. These solutions play the same role among all the variations of solutions of the $\mathrm{KZ}$ equation, such as the well-known sawtoothlike wave for the equation of the simple waves (Riemann's equation). [Work supported by CRDF.]

FRIDAY AFTERNOON, 26 JUNE 1998

FIFTH AVENUE ROOM (W), 1:00 TO 3:45 P.M.

\title{
Session 5pPP
}

\section{Psychological and Physiological Acoustics: Processing, Location, Distance and Motion}

\author{
Armin Kohlrausch, Chair \\ Institute for Perception Research, P.O. Box 513, NL-5600MB Eindhoven, The Netherlands
}

Contributed Papers

1:00

1:15

5pPP1. Binaural coherence and the localization of sound in rooms. William M. Hartmann, Zachary A. Constan (Phys. and Astron., Michigan State Univ., East Lansing, MI 48824), and Brad Rakerd (Michigan State Univ., East Lansing, MI 48824)

The absorption coefficient of materials tends to decrease with decreasing frequency. Therefore, the ratio of direct to reverberant sound is normally least favorable at low frequency, and binaural coherence tends to be a minimum. However, the ability to localize sounds using interaural time differences (ITD) in the steady-state waveform requires binaural coherence, especially at low frequency. Lateralization experiments, using a forced-choice staircase technique, indicate that adequate localization based on ITD requires binaural coherence of at least 0.2. Measurements of binaural coherence in rooms find that binaural coherence is often less than that, which means that ITD cues can only be used on the transient portions of a signal. By contrast, interaural level differences are perceptually useful at high frequencies and increasingly dominate the localization of steadystate sounds as the environment becomes more reverberant. [Work supported by the NIDCD, Grant No. DC00181.]
5pPP2. Interaural correlation sensitivity. John F. Culling, Matthew Spurchise, and H. Steven Colburn (Dept. of Biomed. Eng., Boston Univ., 44 Cummington St., Boston, MA 02215)

Sensitivity to differences in interaural correlation was measured as a function of reference interaural correlation and frequency (250 to 1500 $\mathrm{Hz}$ ) for narrow-band-noise stimuli (1.3 ERBs wide) and for the same stimuli spectrally fringed by a broadband correlated noise. $\mathrm{d}^{\prime}$ was measured for two-interval discriminations between fixed pairs of correlation values, and these measurements were used to generate cumulative $\mathrm{d}^{\prime}$ versus correlation curves for each stimulus frequency and type. The perceptual cue reported by subjects was perceived as intracranial breadth for narrow-band stimuli (wider image for lower correlation) and loudness of a whistling sound heard at the frequency of the decorrelated band for the fringed stimuli (louder for lower correlation). At low correlations, sensitivity was greater for fringed than for narrow-band stimuli at all frequencies, but at higher correlations, sensitivity was often greater for narrowband stimuli. For fringed stimuli, cumulative sensitivity was greater at low frequencies than at high frequencies, but listeners produced varied patterns for narrow-band stimuli. The forms of cumulative $\mathrm{d}^{\prime}$ curves as a function of frequency were interpolated using an eight-parameter fitted function. 
Such functions may be used to predict listeners' perceptions of stimuli that vary across frequency in interaural correlation. [Work supported by MRC and NIDCD Grant No. DC00100.]

\section{$1: 30$}

5pPP3. Modeling the "effective" binaural signal processing. Carsten Zerbs (Graduiertenkolleg Psychoakustik, Univ. of Oldenburg, P.O. Box 2503, 26111 Oldenburg, Germany,cz@medi.physik.uni-oldenburg.de), Torsten Dau, and Birger Kollmeier (Univ. of Oldenburg, 26111 Oldenburg, Germany)

This paper describes a quantitative model for binaural processing in the auditory system. To overcome shortcomings of several proposed models, a monaural stage is employed for signal preprocessing [Dau et al., J. Acoust. Soc. Am. 99, 3615-3622 (1996)], which is able to simulate a large variety of monaural detection data. A binaural processing stage is added following Durlach's EC model which is also a simple functional unit with a small set of constant parameters unit. The decision algorithm for both the monaural and the binaural channel is realized as an optimal detector in the same way as described in the monaural model. In the simulations the whole adaptive measurement procedure with the actual signals is realized. The model describes both monaural as well as binaural thresholds. The typical binaural masking experiments with the following stimulus parameters and their combinations were examined: signal frequency, signal duration, masker bandwidth, interaural masker and signal phase, interaural masker correlation, and delay in simultaneous and forward masking conditions. The model describes the experimental data with an accuracy of a few $\mathrm{dB}$ with some exceptions. Model predictions are discussed and compared to the performance of human observers and to predictions of other binaural models.

\section{$1: 45$}

5pPP4. The influence of masker variability on estimates of monaural and binaural critical bandwidths. Armin Kohlrausch, Steven van de Par, and Jeroen Breebaart (IPO Ctr. for Res. on User-System Interaction, P.O. Box 513, NL-5600 MB Eindhoven, The Netherlands)

If integration bandwidths are derived from band-widening masking experiments, binaural estimates are usually a factor 2 to 3 larger than monaural estimates, at least at high levels. It is proposed that this difference does not reflect "different critical bandwidths," but that it is a consequence of detection statistics. In monaural experiments, the variability in the masker energy limits detectability of the signal. Therefore with increasing subcritical bandwidths, the $\mathrm{S} / \mathrm{N}$ ratio at threshold decreases. In binaural NoS $\pi$ experiments, the $\mathrm{S} / \mathrm{N}$ ratio at subcritical bandwidths remains constant because the masker correlation is always 1 without uncertainty. Due to this absence of external variability in the masker correlation, detection must be limited by internal noise. Binaural detection in narrowband conditions can gain from the spread of excitation across several critical bands, assuming that the internal noises are uncorrelated. The wider binaural critical band is then caused by masking the off-frequency spread through masker components outside the central critical band (for binaural model simulations, see Breebaart et al.). In this study it is shown that this scheme also applies to monaural frozen-noise maskers, which do not have external variability. In agreement with results from binaural experiments, the integration bandwidths increase considerably with masker level.

\section{2:00}

5pPP5. Modeling spectral integration in binaural signal detection. Jeroen Breebaart, Steven van de Par, and Armin Kohlrausch (IPO Ctr. for Res. on User-System Interaction, P.O. Box 513, NL-5600 MB Eindhoven, The Netherlands)

Estimates of critical bandwidths in binaural experiments vary considerably across experimental conditions. For masking experiments using signals of varying bandwidths (i.e., spectral integration), and experiments with a frequency-dependent interaural masker correlation, bandwidth estimates agree with those from monaural experiments. However, if auditory bandwidths are estimated from binaural band-widening masking experiments, the effective bandwidth is usually a factor of 2 to 3 times larger than monaural estimates. One often ignored detail is that this difference between monaural and binaural estimates decreases with decreasing masker level [Hall et al., J. Acoust. Soc. Am. 73, 894-898 (1983)]. Using the binaural model described by Breebaart et al., we have simulated the binaural experiments described above. It can be shown that, without any parameter change in the model, basically all relevant conditions can be modeled accurately, including the band-widening and spectral integration experiments. In agreement with the scheme discussed by Kohlrausch et al., detection in binaural narrow-band-noise conditions is improved by analyzing the internal representation in several adjacent filters. It is concluded that the absence of this detection advantage in monaural randomnoise conditions is the primary cause for the so-called "wider binaural critical band."

\section{2:15}

5pPP6. The role of the head related transfer function low-frequency component for sound localization on a median plane. Motoki Yairi, Yasuko Kuroki, Masayuki Morimoto (Environ. Acoust. Lab., Faculty of Eng., Kobe Univ., Nada, Kobe, 657 Japan), and Kazuhiro Iida (AVC Res. Lab. Matsushita Comm. Ind., Saedo, Tsuzuki, Yokohama, Japan)

Asano et al. [J. Acoust. Soc. Am. 88, 159-168 (1990)] demonstrated that localization is not accurate for the simulation of sound sources on the median plane when the low-frequency component of head related transfer functions (HRTFs) are smoothed. In the present localization experiment, the low- and high-frequency components of white-noise stimuli were separated at $\mathrm{fc}=4.8 \mathrm{kHz}(-48 \mathrm{~dB} / \mathrm{oct}$.). Those components were independently presented from different directions. Results show that the sound image is localized in the direction from which the high-frequency component is presented, regardless of the direction of low-frequency component, showing that the low-frequency component has no evident effect on the sound localization on a median plane. Based on the results of these two experiments, it is concluded that only the high-frequency component of an HRTF provides spectral cues for sound localization on a median plane. However, changing the low-frequency component of the HRTF, which is unlikely to naturally occur in sound localization on a median plane, may interfere with accurate localization.

\section{2:30-2:45 Break}

\section{$2: 45$}

5pPP7. The AUDIS catalog of human HRTFs. Jens Blauert, Marc Brueggen (Ruhr Univ., D-44780 Bochum, Germany, blauert@ika.ruhr-uni-bochum.de), Adelbert W. Bronkhorst, Rob Drullman (TNO NL-3769 ZG Soesterberg), Gerard Reynaud, Lionel Pellieux (Sextant Avionique, F-33166 Saint-Medard-en-Jalles), Winfried Krebber, and Roland Sottek (Head Acoust. GmbH, D-52134, Herzogenrath, Germany)

In the European-Union-funded project AUDIS (AUditory DISplay), a multipurpose auditory display for 3D hearing applications is being developed. The main goal is to develop a special sound generator in combination with an auditory-symbol database that can, for example, be used in the avionic and automotive industry. As the project relies heavily on binaural technology and, hence, on the availability of reliable human HRTF data, a special program for collecting data has been undertaken. In the course of this program, round-robin tests have been performed in four different laboratories. Differences in the data across the different laboratories have been analyzed. The analysis resulted in recommendations for common measuring procedures to be applied. A catalog of HRTFs has then been collected and perceptual verification tests have been performed. It is planned to make the catalog publicly available. In the presentation, the measuring methods applied and the contents of the catalog will be discussed. 
the intermixed condition, subjects knew that $80 \%$ of signals would be presented from one location (the primary location) and $20 \%$ would be presented from the other (the probe location). In the second, subjects knew that all signals would be presented from either the primary or probe location. For each subject the primary location was 0 degrees for half the sessions and 90 degrees for the others. Each function was measured using a two-interval forced-choice task and five signal levels ranging from 7.5 $\mathrm{dB}$ above to $4.5 \mathrm{~dB}$ below the subject's previously estimated threshold. For primary location signals, average functions for the two conditions were indistinguishable. For probe location signals, however, functions differed, reflecting significantly poorer detection performance in the intermixed condition $[F(1,7)=3 \mathrm{D} 22.38, p<0.01]$. This uncertainty effect provides support for the notion that spatially tuned analyzers exist within the auditory system.

\title{
3:30
}

5pPP10. Detection of Doppler-like signals. Mark A. Ericson and Lawrence L. Feth (The Ohio State Univ., Dept. of Speech and Hearing Sci., Columbus, $\mathrm{OH} 43221$ )

Moving sounds induce a Doppler shift of frequency in direct proportion to a sound source's velocity. Concurrent with the frequency change is a change in intensity related to the source's velocity and distance from the listener. These simultaneous changes in frequency and intensity may be audible for any moving sound source. Jorasz and Dooley [Arch. Acoust. 21, 149-157 (1996)] modeled the physical effects of constant velocity motion on a sound source's frequency and intensity as observed at a single point in space. Three linear trajectories of a sound source were simulated over headphones for different minimal distances between the moving sound source and observer. These minimal distances were 1, 10, and 100 $\mathrm{m}$. An adaptive tracking procedure was used to determine detection thresholds for velocity induced changes in frequency and intensity. Intensity and frequency were varied independently as well as together. A series of experiments was conducted to test a perceptual model of these Doppler effects.

FRIDAY AFTERNOON, 26 JUNE 1998

WEST BALLROOM B (S), 1:00 TO 3:00 P.M.

\section{Session 5pSA}

\section{Structural Acoustics and Vibration: Vibrations of Complex Structures II}

\author{
Noureddine Atalla, Cochair \\ GAUS Mechanical Engineering, Universite de Sherbrooke, Sherbroooke, QC JIK 2R1, Canada \\ Paul E. Barbone, Cochair \\ Department of Aerospace and Mechanical Engineering, Boston University, 110 Cummington Street, \\ Boston, Massachusetts 02215
}

Contributed Papers

1:00

5pSA1. Measurement and analysis of the structure-borne sound of a fan installation: Evaluation of source signal filtering techniques and rotational degrees of freedom. Michael A. Sanderson, Lars Ivarsson (Dept. of Appl. Acoust., Chalmers Univ. of Technol., 41296 Gothenburg, Sweden, mike@ta.chalmers.se), and Andrej G. Troshin (Krylov Shipbuilding Res. Inst., St. Petersburg, Russia)

The structure-borne sound characteristics of a commonly found fan installation were examined through measurements and further analyses in the frequency range up to $500 \mathrm{~Hz}$. The focus was to determine the prediction improvements by including the rotational degrees of freedom. Free velocities of the fan were measured in five degrees of freedom (in-plane rotation or twisting motion were neglected), while fan mobilities were measured and foundation mobilities were predicted in three degrees of freedom: a translational and two rotational, those resulting in bending motion. The two in-plane translations were considered by transforming their motion to rotations resulting in moment excitations on the foundation. Measurements were also made of the corresponding isolators' stiffness values. Comparisons are made with power measurements of the complete system. It was found that an unsatisfactory moment excitation resulted in one of the rotational degrees of freedom while employing a twin shaker arrangement when determining the fans' mobilities. Therefore, a source signal filtering technique was applied to improve the mea- 
surement. Comparisons are made with the originally measured mobilities. [This work has been supported by the Swedish Council for Building Research (BFR) through Contract 950192-8 and the Swedish Research Council for Engineering Sciences (TFR) through Contract 95-160.]

\section{$1: 20$}

5pSA2. Modeling the vibration behavior of an induction motor. Chong Wang and Joseph C. S. Lai (Acoust. and Vib. Unit, Univ. College, Univ. of New South Wales, Australian Defence Force Acad., Canberra, ACT 2600, Australia, c-wang@adfa.oz.au)

With the advent of power electronics, variable speed induction motors are finding increased use in industries because of their low cost and savings in energy usage. However, the acoustic noise emitted by the motor increases due to switching harmonics introduced by the electronic inverters. Consequently, the vibro-acoustic behavior of the motor structure has attracted more attention. In this paper, considerations given to modeling the vibration behavior of a $2.2-\mathrm{kW}$ induction motor will be discussed. By comparing the calculated natural frequencies and the mode shapes with the results obtained from experimental modal testing, the effects of the teeth of the stator, windings, outer casing, slots, endshields, and support on the overall vibration behavior are analyzed. The results show that when modeling the vibration behavior of a motor structure, the laminated stator should be treated as an orthotropic structure, and the teeth of the stator could be neglected. As the outer casing, endshields, and the support all affect the vibration properties of the whole structure, these substructures should be incorporated in the model to improve the accuracy.

\section{1:40}

5pSA3. Model-based shaping of vibroacoustic properties of gears. Stanislaw Radkowski (Vibroacoustic Lab. IPBM PW, 02-524 Poland, Warszawa, Narbutta 84)

The work undertakes an issue of estimating and predicting the level and structure of the vibroacoustic signal generated by a pair of toothed wheels and propagated by the structure of the gear's housing. Assuming that the basic role in transmission of such diagnostic information is played by the phenomena of amplitude and phase modulation of the vibroacoustic signal, it is pointed to the significance of the signal's nonlinear components. For that reason in this work a model of a signal in the form of a Volterra series is assumed, which enables examination of the influence that disturb nonlinear components. On the basis of exemplary analysis of the contact conditions disturbances, the possibility of the generation of a bilinear component is presented. What serves this purpose is the model of nonlinear and instantaneous contact. Simultaneously problems of selection of signal analysis methods that enable realization of assumed aims are presented. Emphasizing the fact that defect development does not always lead to a significant growth of the vibroacoustic signal's power, the importance of methods of higher-order spectral analysis like bispectrum and bicepstrum has been shown.

\section{2:00}

5pSA4. Experimental identification of mechanical joint dynamic properties. Olivier Chiello, Franck C. Sgard (LASH, DGCB URA CNRS 1652, ENTPE-Rue Maurice Audin, 69518 Vaulx-en-Velin, Cedex, France, olivier.chiello@entpe.fr), and Noureddine Atalla (GAUS, Univ. de Sherbrooke, QC J1K 2R1, Canada)

Large structures are often composed of substructures connected through mechanical joints. The dynamic behavior of these joints is generally difficult to characterize. In this paper, a method for identifying dynamic joint characteristics is presented. Joint properties are extracted di- rectly from measured frequency responses of the assembled structure. Measurement points are not taken on the interface between the structure components. This feature is of practical importance since it is often difficult to directly attach a transducer on the joint. The method only requires the knowledge of the frequency responses of the substructures taken separately. These responses can be either measured, analytically calculated, or simulated by FEM. The presented approach allows for the determination of the joint properties as a function of frequency. The joint properties extracted from one assembled structure can be used to predict the behavior of another structure assembled with the same joint condition. The applicability of the proposed method is investigated by numerical simulations. Errors in simulated frequency responses are introduced to investigate the robustness of the method. It is shown that the accuracy of the identified results is good.

\section{2:20}

5pSA5. The vibroacoustical analysis of the teeming crane. Jerzy W. Wiciak, Ryszard J. Panuszka, and Marek A. Iwaniec (Structural Acoust. and Intelligent Mater. Group, Tech. Univ. of Mining and Metallurgy, Al. Mickiewicza 30, 30-059 Cracow, Poland)

The present article considers the vibration analysis of the teeming crane (lifting capacity $Q=160$ tons) and noise caused by working units of the crane. The vibroacoustical investigations and theoretical analysis have been applied to the main bridge with box-trapezoid cross section. In the article there is shown a comparison of the vibration acceleration and dynamic stress that have been measured in real structure with calculations obtained by means of the finite element method. The correct and incorrect (two-stage) reduction roll gear running also was considered. The significant influence of the incorrect reduction roll gear running on the dynamic parameters was noticed. The noise caused by working units of the crane has been presented for three operating conditions: when the tilting ladle was descending, when it was hoisting, and pass-by load running of the teeming crane. [Work supported by TUMM, Cracow, Poland.]

\section{2:40}

5pSA6. Metal and composite aircraft panels: Selection of parameters optimal from the viewpoint of acoustic fatigue lifetime. Nikolay I. Baranov, Sergey N. Baranov, Lev S. Kuravsky, and Konstantin P. Zhukov ("RusAvia" Res. Group, Mikoyan Design Bureau, 6 Leningradskoe Shosse, 125190 Moscow, Russia)

Optimization techniques to ensure synthesis of stiffened and laminated composite panels subjected to acoustic excitation are presented. The criteria for optimization are established to balance the structure weight and fatigue life. Test spectrum of loading pressure fluctuations is in use, with the parameters of pressure correlation ensuring the greatest response. For stiffened structures, stress spectra and corresponding root-mean-square values in points of maximum response are computed by means of special software to draw the special final diagrams that connect nondimensional structure parameters and optimization criteria. These diagrams are then used to select optimal parameters of reinforcement. For composite structures, correlation of the nondimensional parameters representing structure stress level, acoustic load level, dominant frequency of vibration, and panel anisotropy is computed by the same technique. The functions computed are later used to estimate how the criterion for optimization depends on the number of layers, lay-up sequence, and the directions of fibers. The approach proposed allows aerospace designers to select and motivate their decisions having increased confidence in the development and modifications of these structures. 


\title{
Session 5pSC
}

\section{Speech Communication: Segmental Phonetics, L2, and Cross Language Studies (Poster Session)}

\author{
Joseph S. Perkell, Chair \\ Massachusetts Institute of Technology, Room 36-591, 50 Vassar Street, Cambridge, Massachusetts 02139-4307
}

\section{Contributed Papers}

\begin{abstract}
All posters will be on display from 1:00 p.m. to 5:00 p.m. To allow contributors an opportunity to see other posters, contributors of odd-numbered papers will be at their posters from 1:00 p.m. to 3:00 p.m. and contributors of even-numbered papers will be at their posters from 3:00 p.m. to 5:00 p.m.
\end{abstract}

\begin{abstract}
5pSC1. Do nasalized fricatives exist? John J. Ohala (Dept. of Linguist., Univ. of California at Berkeley, 2337 Dwinelle Hall \#2650, Berkeley, CA), Maria-Josep Sole (Univ. Autonoma de Barcelona, 08193 Bellaterra, Barcelona, Spain), and Goangshiuan S. Ying (Univ. of California at Berkeley, Berkeley, CA)
\end{abstract}

It has been claimed that nasalized fricatives, even sibilant fricatives, exist in some languages. To assess this claim we sought to discover how much velopharyngeal opening can be present before the acoustic and auditory characteristics of fricatives are affected. Two trained phoneticians produced steady-state voiced and voiceless strong and weak fricatives. The back pressure was intermittently bled with a tube of varying diameter (and thus impedance) inserted into the speaker's mouth via the buccal sulcus and the gap behind the back molars, simulating nasal leakage. Intraoral pressure $(P o)$ was sampled via a catheter inserted into the pharynx through the nose. The changes in amplitude and quality of frication were analyzed acoustically. We found that (1) a vent area of about $18 \mathrm{~mm}^{2}$ caused auditorily and acoustically noticeable lessening of fricative energy, e.g., sibilants sounded more like nonsibilants, (2) for a given vent aperture, $P o$ was diminished less for voiceless than voiced fricatives (because the voiced fricatives have higher upstream impedance at the glottis) and consequently the same vent aperture impaired the amplitude and quality of frication of voiced fricatives more than voiceless ones. Thus the aerodynamic requirements for fricatives seem to be relatively narrow and unforgiving. [Research supported by Committee on Research, UCB, and by DGICYT, Spain, PB 96-1158.]

5pSC2. Aerodynamic characteristics of trills. Maria-Josep Sole (Dept. of English, Univ. Autonoma de Barcelona, 08193 Bellaterra, Barcelona, Spain, sole@cc.uab.es), John J. Ohala, and S. Goangshiuan Ying (Univ. of California at Berkeley, Berkeley, CA 94720-2650)

What are the aerodynamic conditions required for trills? To find out we had two subjects produce steady-state voiced, voiceless, and ejective alveolar trills. The backpressure during trills was intermittently bled with a tube of varying diameter (and thus impedance) inserted in the speaker's mouth via the buccal sulcus and gap behind the back molars. Intraoral pressure was measured via a catheter inserted into the pharynx through the nose. The variation, impairment, or extinction of trilling as a function of gradual decrease in intraoral pressure was analyzed acoustically. It was found that (1) bleeding the oro-pharyngeal pressure by $2 \mathrm{~cm} \mathrm{H}_{2} \mathrm{O}$ impaired sustained trilling; (2) the minimum $P o$ required to sustain tongue-tip vibration is lower than that required to initiate it; (3) extinction (and reinitiation) of trilling generally results in a fricative; (4) the range of $P o$ variation for trills is narrower than that for fricatives; (5) voiceless and ejective trills are significantly less affected by venting the backpressure than voiced trills. The behavior of trills in varying aerodynamic conditions accounts for observed phonological patterns: final trill devoicing, alterna- tion between trills and fricatives, co-occurrence of trilling and frication, and limited distribution of trills. [Work supported by DGICYT, Spain, PB 96-1158, and by Committee on Research, UCB.]

5pSC3. Motor equivalence in the production of $/ \int /$. Joseph S. Perkell, Melanie L. Matthies, and Majid Zandipour (Speech Commun. Group, R.L.E., MIT, Rm. 36-511, 50 Vassar St., Cambridge, MA 02139)

To explore the idea that speech motor goals are acoustic targets, upper lip protrusion and tongue blade fronting were examined in the sibilant $/ \mathrm{J} /$ for evidence of motor equivalence in eight speakers of American English. Positive correlations across multiple repetitions of /J/ (motor equivalence) would occur if the upper lip compensated with more protrusion when the tongue blade was further forward and vice versa. This motor equivalence would serve to maintain an adequate front cavity size for good acoustic separation from /s/ and enhance the acoustic stability of / $/ \mathrm{J}$. It was hypothesized that motor equivalence would be found among less prototypical tokens, as elicited in a "clear+fast" speaking condition. Acoustic spectral analyses indicated excellent acoustic separation between the two sibilants, even in the "clear+fast" condition. There were significant positive correlations of the tongue-blade and upper lip movements for $28 \%$ of all possible cases, with considerable individual variation. When motor equivalence could be compared across the speaking condition, the tokens produced with the motor equivalent pattern were acoustically less prototypical. No occurrences of negative movement correlations were found. One possible source of individual variation may be the use of saturation (quantal) effects, which could also enhance acoustic stability. [Work supported by NIH.]

5pSC4. Further results on the perception of place coarticulation in Taiwanese stops. Shu-hui Peng and Terrance Nearey (Dept. of Linguist., Univ. of Alberta, 4-32 Assiniboia Hall, Edmonton, AB T6G 2E7, Canada, speng@gpu.srv.ualberta.ca.)

Previous study of place coarticulation using logistic discrimination [Peng and Nearey, J. Acoust. Soc. Am. 101, 3110(A) (1997)] showed that $87 \%$ of Taiwanese word-final dental or velar stops in stop consonant clusters $[\mathrm{CV}$ stop1-stop2V(C)] spoken in various speech rates were correctly classified based on formant transitions from the preceding vowel. This automatic classification was sigificantly (though only moderately) correlated with categorization by Taiwanese speakers in a concept formation test. An earlier study [Peng (1996)] also showed that there was not a consistent correlation between Taiwanese listeners' categorization of the stops and patterns of gestural overlapping shown in EPG measurements. To avoid complication involving listeners' difficulty with the concept formation task and the influence of native speakers' lexical knowledge, the present study examined the perception of the stops by phonetically trained 
listeners in a different experimental paradigm. Listeners were asked to identify the consonant clusters (tt, kk, tk, kt) from the excised first word in a four-way forced choice identification test. The phonetic judgments of these listeners will be compared with the categorization by Taiwanese speakers, the results of discriminant analysis, and EPG measurements.

5pSC5. Ejectives in Tanana Athabaskan. Siri G. Tuttle (Univ. of Washington, Dept. of Linguist., Box 354340, Seattle, WA 98195, iris@u.washington.edu)

Ejectives in Athabaskan languages have been investigated phonetically by Lindau (1984), McDonough and Ladefoged (1993), in Navajo, and by Davis and Hargus $(1994,1996)$ in Babine/Witsuwit'en. In the latter two investigations, three different types of production were found associated with glottal consonants, only one of which showed the classic ejective pattern with a silent period. Examination of samples of Tanana Athabaskan recorded in 1961, 1962, 1974, and the early 1990s found similar variation in samples of this Alaskan Athabaskan language. There appeared, however, to be no examples of ejectives without silent periods in the oldest samples (1961 and 1962). This suggests the hypothesis that the ejective without silent period is an innovation in the last generation of Tanana speakers. Recordings analyzed include a set of standardized word lists recorded by McKennan in 1962 with speakers of Minto, Chena, and Salcha dialects; texts of Teddy Charlie (Minto) recorded by Michael Krauss in 1962; and 3keywords2 recordings of Isabel Charlie (Minto) and Eva Moffit (Salcha) recorded by James Kari and Siri Tuttle in 1991. [This work has been supported by NSF.]

5pSC6. Perception of synthesized Hindi geminate and cluster sounds. Nisheeth Shrotriya and Shyam S. Agrawal (Speech Technol. Group, CEERI Ctr., CSIR Complex, Hillside Rd., New Delhi-12, India)

This paper describes the various differences in acoustic parameters of geminate, nongeminate, and cluster sounds of Hindi, which can be used for their perceptual distinction. Words containing these sounds were synthesized using a PC-based Klatt synthesizer, which uses a set of about 60 parameters. For distinction between geminate and nongeminate sounds, the parameters such as duration of the plosive gap and preceding vowel, and the formant frequencies and intensity of the burst are important but the closure duration plays the most significant role. This difference is of the order of 1:1.5. However, if the closure duration is reduced to $50 \%$ or less, the unvoiced sounds are perceived as voiced sounds. In the case of the cluster (-C1C2-) words containing stop consonants, when $\mathrm{C} 1$ and $\mathrm{C} 2$ have the different places of articulation, a burst is observed in the middle of the closure. It was found that this burst has no perceptual effect when it was moved to the left and right positions adjacent to the vowels. Even the removal of the burst has no perceptual effect.

5pSC7. Integration of acoustic cues in Spanish voiced stops. Sergio Feijóo, Santiago Fernández, and Ramón Balsa (Dpto. Física Aplicada, Fac. de Física, 15706, Santiago, Spain, fasergio@uscmail.usc.es)

In Spanish, voiced stops in initial position usually show the presence of a voice bar before the release burst. In this study, the perception of word-initial voiced stops when the voice bar is removed is examined. Nine men and nine women pronounced 15 Spanish words in which the initial consonant was $/ \mathrm{b} /, / \mathrm{d} /$, or $/ \mathrm{g} /$ in combination with the vowels $/ \mathrm{a} /, / \mathrm{e} / \mathrm{l} / \mathrm{i} /$, $/ \mathrm{o} /, / \mathrm{u} /$. Seventeen subjects with normal hearing heard the stops in two conditions: (1) whole voice bar $+102.4 \mathrm{~ms}$ of signal from burst onset; (2) $102.4 \mathrm{~ms}$ from burst onset. In condition (2), tokens were perceived as the correct stop, as its voiceless counterpart, or as an ambiguous signal. Removing the voice bar caused $37 \%$ of $/ \mathrm{b} /$ tokens to be classified as $/ \mathrm{p} / ; 41 \%$ of $/ \mathrm{d} / \mathrm{s}$ to be classified as $/ \mathrm{t} / \mathrm{s}$; and $19 \%$ of $/ \mathrm{g} / \mathrm{s}$ to be classified as $/ \mathrm{k} / \mathrm{s}$. The change in voicing characteristics was significantly more marked for women. The results indicate that the integration of the voice bar with the burst and the vowel plays a relevant role in the perception of voicing in voiced stops, especially in women's speech, smoothing the dynamic frequency characteristics of burst and vowel.

5pSC8. Acoustic properties of English fricatives. Allard Jongman, Joan Sereno, Ratree Wayland, and Serena Wong (Cornell Phonet. Lab., Dept. of Modern Lang., Cornell Univ., Ithaca, NY 14853)

A fundamental issue in speech research concerns whether distinctions in terms of place of articulation are more succesfully captured by local (static) or global (and/or dynamic) properties of the speech signal. While most studies of place of articulation have investigated stop consonants, it is uncertain whether these properties can successfully classify fricatives. In the present study, both static and dynamic metrics were used to investigate place of articulation in fricatives. Static metrics include spectral peak location, noise duration, and noise amplitude; dynamic metrics include relative amplitude, locus equations, and spectral moments. Twenty speakers produced the fricatives /f, v, $\theta, \delta, s, z, \int, 3 /$ followed by the vowels /i, e, æ, a, o, u/. Preliminary results based on ANOVA and discriminant analysis suggest that all metrics could distinguish nonsibilant /f, v, $\theta, \delta /$ from sibilant /s, $\mathrm{z}, \int, 3 /$. In addition, spectral moments separated /s, z/ from / $\int, 3 /$ with about $95 \%$ accuracy and /f, v/ from / $\theta$, ठ/ with about $65 \%$ accuracy while locus equations only served to separate labiodental /f, v/ from all other fricatives. Accuracy of the metrics will also be compared to human perception data. [Work supported by NIH.]

5pSC9. Role of $F 1$ in the perception of voice offset time as a cue for preaspiration. Jörgen Pind (Dept. of Psych., Univ. of Iceland, Oddi, IS-101 Reykjavík, Iceland, jorgen@ rhi.hi.is)

Preaspiration, an [h]-like sound inserted between a vowel and a following stop consonant, can be cued by voice offset time (VOffT), a speech cue which is a mirror image of voice onset time (VOT). Previous research has shown VOffT to be an effective cue for preaspiration and also that the perception of VOffT differs from the perception of VOT in that the former cue is much more sensitive to the duration of a preceding vowel than is VOT to the duration of a following vowel [J. Pind, Q. J. Exp. Psychol. 49A, 745-764 (1996)]. The hypothesis has been put forward that this is due to the fact that preaspiration in Icelandic follows a phonemically short vowel, whereas aspiration can precede either a long or a short vowel. Vowel quantity will therefore affect phoneme boundaries for VOffT, not for VOT. This hypothesis was confirmed in experiments where vowel spectra were used to manipulate quantity rather than vowel duration. The present paper reports experiments investigating whether this effect could possibly be mediated through the effect of $F 1$ on perceived voicing. [Supported by Icelandic Science Foundation and University of Iceland.]

5pSC10. Vowel and consonant durations as cues for quantity in Icelandic. Jörgen Pind (Dept. of Psych., Univ. of Iceland, Oddi, IS-101 Reykjavík, Iceland, jorgen@rhi.hi.is)

Icelandic distinguishes short and long consonants and vowels in stressed syllables. Phonologically, these show complementary quantity in that a short vowel is followed by a long consonant and vice versa. Perceptual experiments have shown that the ratio of vowel to consonant duration is the major cue for quantity, although the vowel spectrum can override the relational cue in those cases where there is a marked difference in the spectral character of the long and short vowels [J. Pind, Scand. J. Psychol. 37, 121-131 (1996)]. Measurements will be reported of vowel and consonant durations in stressed syllables in words spoken repeatedly in sentence frames and in longer connected texts. The utterances were read at different speaking rates. Two questions are of major interest in this study: (1) To what extent do vowel and consonant durations in different environments support a relational cue for quantity? and (2) Is the dura- 
tional cue weaker in those cases where the spectra of short and long vowels provide another cue for quantity? [Work supported by the Icelandic Science Foundation and University of Iceland.]

5pSC11. Timing effects on successive alveolar consonants in Swedish. Robert Bannert and Peter E. Czigler (Dept. of Phonet., Umeå Univ., S-90187 Umeå, Sweden)

The duration of a consonant is potentially affected by various factors related to the phonetic nature of the segment and its phonetic environment. The present study addresses the concurrent effects of prominence, syllable structure, and manner of articulation in intervocalic /s/+alveolar consonant $/ \mathrm{t}, \mathrm{n}, \mathrm{l}$, and alveolar consonant $+/ \mathrm{s} /$ clusters in Swedish. Six real words, imbedded in a carrier sentence, were repeated five times with, and five times without, focal accent by ten native speakers of standard Swedish. Results show that the duration of a cluster, and the duration of its constituent consonants, are longer with focal accent than without focal accent. Consonant clusters with $/ \mathrm{s} /$ as the first member do not differ in duration from consonant clusters with $/ \mathrm{s} /$ as the second member. Clusters containing an alveolar stop are shorter than clusters with an alveolar nasal or alveolar liquid. The duration of $/ \mathrm{s} /$ is always longer than the duration of any adjacent alveolar stop, nasal or liquid. A number of interactions was found between prominence, cluster structure, and manner of articulation, indicating clear differences between the patterns of cluster internal timing for the investigated cluster pairs. These differences will be presented, compared with data on American English, and discussed.

5pSC12. Acoustic and perceptual effects of vowel length on voice onset time in Thai stops. Chutamanee Onsuwan and Patrice S. Beddor (Prog. in Linguist., Univ. of Michigan, Ann Arbor, MI 48109, beddor@umich.edu)

In Thai, vowel length (e.g., [pan]-[paan]) and unaspirated-aspirated stop (e.g., [pan]-[ph $\left.\mathrm{p}^{\mathrm{h}}\right]$ ) distinctions are contrastive; this study examines possible acoustic and perceptual interactions between these temporal properties. VOT and vowel duration were measured for five repetitions of 40 (near-) minimal word pairs beginning with aspirated or unaspirated bilabial, alveolar, or velar stops followed by long or short vowels, produced by three native Thai speakers. Although VOT for velars was slightly longer (about $5 \mathrm{~ms}$ ) before long than before short vowels, the overall acoustic picture across places of articulation showed no systematic effect of vowel length. To test for perceptual influences of contrastive vowel length on VOT, a 15-step 0-90 ms VOT continuum was created by editing the aspiration portion of naturally produced [ $\left.\mathrm{p}^{\mathrm{h}} \mathrm{aan}\right]$. The VOT continuum was spliced onto the vowel portion of four different natural tokens, [pan], [paan], [ $\left.\mathrm{p}^{\mathrm{h}} \mathrm{an}\right]$, and $\left[\mathrm{p}^{\mathrm{h}} \mathrm{aan}\right]$, creating four continua, two with long vowels and two with short. Identification tests were presented to 18 native Thai listeners. Responses to the continua created from the originally unaspirated tokens ([pan], [paan]) showed robust effects of vowel length: the long vowel continuum elicited more aspirated $\left(\left[\mathrm{p}^{\mathrm{h}}\right]\right)$ responses. A compensatory account of the perceptual findings is offered. [Work supported by NSF.]

5pSC13. Perception of glottalized consonants in Babine/Witsuwit'en. Katharine Davis and Sharon Hargus (Dept. of Linguist., Univ. of Washington, Box 354340, Seattle, WA 98195-4340)

Previous studies of Babine/Witsuwit'en have shown speaker differences in the production of glottalized stops [Davis and Hargus, J. Acoust. Soc. Am. 100, 2690 (1996)]. The present study investigates native listeners' perception of the glottal/nonglottal contrast. Ten subjects were presented with 16 naturally produced minimal pairs contrasting glottalized and voiceless unaspirated stops or affricates. These tokens, interspersed with 18 control minimal pairs, were presented in random order. Listeners' judgments were recorded on a forced-choice answer sheet. The task was performed twice, once with a male voice and once with a female voice.
Subjects showed greater confusion when identifying members of the glottal/nonglottal contrast than when identifying members of the control contrasts (84\% correct versus $93 \%$ correct). Among control items, voiceless unaspirated and voiceless aspirated pairs were correctly distinguished 97\% of the time. ANOVA results considering all experimental factors (subject, speaker, place of articulation, etc.) will be presented. In addition, acoustic analyses of correctly identified versus confused glottal/nonglottal pairs will be presented. Particular attention will be paid to significant factors (VOT, $F 0)$ from earlier production studies.

5pSC14. A perceptual experiment on Thai consonant types and tones. Rungpat Roengpitya (Univ. of California, Berkeley)

Tonogenesis in Thai arose historically from neutralization of voicing contrast prevocalic consonants: new higher tonal variants after what had been voiceless consonants and new lower variants after the earlier voiced consonants. Today, Thai has again acquired a series of voiced stops and the same allophonic variation in $F 0$ may be observed on the tones following voiced and voiceless stops. How important are these $F 0$ cues to Thai listeners? Can one stage of the tonal multiplication process that shaped Thai tonal systems be demonstrated in the lab? A perceptual experiment was conducted. Six pairs of Thai words with voiced and voiceless initial stops were recorded, spliced at six different points of time, and put into contexts. These tokens were played to 11 native-Thai subjects for judgment as voiced or voiceless. The result showed that listeners tended to identify those syllables with gated-out bursts and closures as voiceless. However, there was a significant success at differentiating voiced and voiceless stops at gates past these points. It remains to be determined whether the cues that facilitate this are $F 0$, vowel amplitude, or voice quality. If $F 0$ plays a role, then this result mirrors the rise of distinctive tones in the Thai history.

5pSC15. The nasal vowels of Iberian Portuguese. Maria Joao C. Galvao (Univ. of Washington, Dept of Linguist., Box\# 354340, Seattle, WA 98195-4340,mgalvao@u.washington.edu)

Previous analysis [Mateus, "Aspectos da Fonologia Portuguesa", (1982)] of Iberian Portuguese nasal vowels has suggested that there are three degrees of nasality, predictable from syllable structure and from the presence or absence of an underlying nasal consonant following the nasal vowel. This study addresses the question of whether vowel quality affects the degree of phonetic nasality in Portuguese. Nasalance measurements of seven native speakers of Portuguese were obtained via a Kay Nasometer 6200, while spectrographic analysis was provided by a Kay CSL 4300B. The present analysis confirms that a trace of the nasal consonant is phonetically present which provides a peak target for the nasal vowel, but the degree of nasality of the vowel itself is solely determined by vowel height: as occurs in other languages with nasal vowels, high vowels have a more constricted oral air passage and hence higher nasalance percentage.

5pSC16. EMG evidence for the automaticity of intrinsic $F 0$ of vowels. D. H. Whalen, Bryan Gick, Masanobu Kumada (Haskins Labs., 270 Crown St., New Haven, CT 06511), and Kiyoshi Honda (ATR Human Information Processing Res. Labs, 2-2 Hikaridai Seika-cho Soraku-gun, Kyoto, 619-02 Japan)

Although vowels can be produced with many $F 0$ values, high vowels' $F 0$ s tend to be higher than low vowels', a tendency found for every language examined. Nonetheless, this "intrinsic $F 0$ " (or $I F 0$ ) has been called a deliberate "enhancement" of the speech signal, aiding vowel height perception. Since $I F 0$ remains constant with the number of vowels in a language, this enhancement seems unlikely. The only positive evidence for it is that activity of the cricothyroid (CT) muscle, which primarily raises $F 0$, is larger for high vowels than for low. The present experiment explores this by having subjects produce vowels at slightly different $F 0$ s, with the differences being similar to the $I F 0$ magnitude for each 
subject. The first subject (of four planned) showed no vowel height difference in CT; further, an analysis factoring out $F 0$ as a covariate showed that high vowels had higher CT activation. Thus different vowels involve different levels of $\mathrm{CT}$ activity for a given $F 0$; previous findings were likely due not to $F 0$ control but to $F 0 /$ vowel quality interactions. Even the EMG evidence, then, renders $I F 0$ an unlikely speech enhancement. Rather, $I F 0$ appears to be an automatic consequence of vowel production. [Supported by NIH Grant DC-02717.]

5pSC17. Interacting spectral and temporal properties in Jamaican Creole and Jamaican English vowel production. Alicia Beckford (Program in Linguist., Univ. of Michigan, 1076 Frieze Bldg., Ann Arbor, MI 48109-1285)

The vowel system of Jamaican Creole has been described as exploiting a contrastive length distinction, wherein long vowels and falling diphthongs contrast with single vowels in identical phonological environments [Wells (1973); Mead (1996)]. For example, /pan/ on, pan, can /tan/ stand /pen/ pen /paan/ to grasp /taan/ turn /pien/ pain. However, the nature of the relative contributions of spectral and temporal differences remains unclear. The interactions between spectral $(F 1 / F 2)$ and temporal properties, and their roles in shaping a three-dimensional vowel space ( $F 1 \times F 2 \times$ length) were examined in an acoustic study of the vowel production of 24 speakers at different points along the creole continuum from Jamaican Creole to Jamaican English. Data suggest that Jamaican Creole and Jamaican English speakers utilize vowel duration differently. It will be demonstrated in this paper that, for the Creole speakers, vowel duration functions to enhance slight spectral distinctions among low central and upper-mid-back vowels /a,o,u/. [Mead, "On the phonology and orthography of Jamaican Creole," J. Pidgin Creole Languages (1996); Wells, Jamaican Pronunciation in London (Blackwell, London, 1973).]

5pSC18. The effect of $\boldsymbol{F 3}$ on the /U-I/ boundary. Matthew R. Buehler and Anna K. Nábělek (Dept. of Audiol. and Speech Pathol., Univ. of Tennessee at Knoxville, 457 S. Stadium Hall, Knoxville, TN 37996)

Boundary locations (50\% response points of identification) were tested for $/ \mathrm{U}-\mathrm{I} / \mathrm{continua}$ for two different values of $F 3$. All formants were steady-state. $F 1=250 \mathrm{~Hz}, F 2$ was varied in 20 steps from 1000 to 2000 $\mathrm{Hz}$, and $F 3=2500$ or $3000 \mathrm{~Hz}$. All stimuli were 200-ms long. The boundaries for the two continua were determined with ten subjects. The boundary for $F 3=2500 \mathrm{~Hz}$ was $1652 \mathrm{~Hz}$ and for $F 3=3000 \mathrm{~Hz}$ was $1526 \mathrm{~Hz}$. The difference between the boundaries was significant. These results indicate that $F 3$ can affect the perception of $F 2$. When $F 3$ was increased the stimuli were identified as /I/ at a significantly decreased value of $F 2$.

5pSC19. The effect of transition slope and transition duration on vowel reduction in $V_{1} V_{2}$ complexes. Pierre L. Divenyi (Experimental Audiol. Res. (151), V. A. Medical Ctr., Martinez, CA 94553) and René Carré (Ecole Nationale Supérieure de Télécommunications, Paris, France)

Digital $V_{1} V_{2}$ vowel pairs were generated using a cascade-parallel formant synthesizer. The duration of $V_{1}$ was $100 \mathrm{~ms}$ and that of a completed $V_{2} 150 \mathrm{~ms}$, while the duration of the transition was varied between 40 and $100 \mathrm{~ms}$, depending on the condition. For each of six English $V_{1} V_{2}$ pairs (/ia/, /ai/, /iu/, /ui/, /ua/, and /au/) 8-12 tokens were obtained by progressively cutting back the $V_{2}$ portion and the vowel transition in 10-ms steps. Separately for each vowel pair, the cut-back tokens were presented to trained listeners who had to indicate their confidence level (using a 4-point scale) that the final portion of the token was, indeed, the vowel $V_{2}$. Since high-confidence $V_{2}$ responses were given not only to tokens that actually reached $V_{2}$, we observed vowel reduction; the degree of vowel reduction (VR) was measured as the severity of the cut-back at which such a misperception occurred at a certain percent of the time. Using this measure, we determined the degree of VR for different durations and velocities of transition. In general, VR was larger for steeper transition slopes and for durations around $80-100 \mathrm{~ms}$. [Work supported by NATO Office of Scientific Research, a grant from N.I.H., and the V.A. Medical Research.]

5pSC20. Acoustic correlates of "devoiced" vowels in standard modern Japanese. J. Kevin Varden (Meiji Gakuin Univ., Dept. of English, Meiji Gakuin Univ., 1-2-37 Shirokane-dai, Minato-ku, Tokyo, 108 Japan, varden@ltr.meijigakuin.ac.jp)

This paper documents the acoustic characteristics of what have been called devoiced vowels in Tokyo Japanese, traditionally attributed to the phonological rule of high vowel devoicing [T. J. Vance, Introduction to Japanese Phonology (1987)]. More recently they have been attributed to articulatory gesture overlap [S-A. Jun and M. E. Beckman, Paper, Annual Meeting of the LSA (1993)]. Current research by the author indicates both processes are consistent with the data. The vowels which have undergone phonological processing, however, are not simply devoiced; they have undergone a process of obstruentization similar to that found in the Turkish language of Uyghur discussed both pedologically [R. F. Hahn, Spoken Uyghur (1991)] and phonologically [E. M. Kaisse, Language 68, 313-332 (1992)]. Specifically, the vowel sites surface as voiceless fricatives at the place of articulation of the preceding voiceless consonant. This is evidenced acoustically by high-frequency frication in spectrograms and a high number of zero crossings. Additionally, formant frequency information is preserved, as evidenced by formant bars in spectrograms and spectral sections, allowing the perception of the original vowels in the face of heavy loss of vocality.

5pSC21. Nasal vibration spectra in vowels. Mechtild Tronnier (Dept. of Linguist. and Phonet., Lund Univ., Helgonabacken 12, S-22362 Lund, Sweden, mechtild.tronnier@ling.lu.se)

An accelerometer microphone attached to the upper part of the nose for measuring pathological nasality has been applied. The advantage of this technique is the easy application, the minor degree of invasiveness, and the minimal speaker distraction. The analysis of the obtained signal has been subject to discussion. Some researchers judged from signal intensity whether speech was produced on a hypernasal level or not. Others calculated the intensity ratio of the accelerometer signal to a voice source signal to eliminate intensity variation. The analysis of the spectral pattern of the accelerometer signal is suggested here. Spectral information in such a signal is a good indicator to reflect the velar port opening, which results in nasal resonance and nasal vibration at the nasal surface. Different vowels induce a different degree of velar opening, which does not necessarily reflect the degree of perceived nasality and measured acoustic resonance. This is due to different nasal and oral impedance of the vowels. Therefore, the spectral patterns of individual vowels in the accelerometer signal are described here. Simultaneously, a fiberendoscopic film has been recorded, showing the velar opening to confirm visually the presence or absence of velopharyngeal coupling.

5pSC22. Perceived vowel quantity in Swedish: Effects of postvocalic voicing. Dawn M. Behne (Norwegian Univ. of Sci. and Technol., 7034 Trondheim, Norway), Peter E. Czigler, and Kirk P. H. Sullivan (Umea Univ., 90187 Umea, Sweden)

Swedish is described as having a distinction between phonologically long and short vowels. This distinction is realized primarily through the duration of the vowels, but in some cases also through resonance characteristics of the vowels. In Swedish, like many languages, vowel duration is also longer preceding a voiced postvocalic consonant than a voiceless one. This study examines the weight of vowel duration and the first and second formant frequencies $F_{1}-F_{2}$ frequencies when distinguishing phonologi- 
cally long and short vowel before a voiceless consonant (experiment 1) and before a voiced consonant (experiment 2). For three pairs of Swedish vowels ([i:]-[I], [o:]-[o], [a:]-[a]) $100 / \mathrm{kVt}$ (experiment 1) and $100 / \mathrm{kVd} /$ (experiment 2) words were resynthesized having ten degrees of vowel duration and ten degrees of $F_{1}$ and $F_{2}$ adjustment. In both experiments listeners decided whether presented words contained a phonologically long or short vowel. Reaction times were also recorded. Results show that vowel duration is a more dominant cue than the first and second formant for distinguishing phonologically long and short vowels. However, for [a:] and [a] the perceptual influence of vowel duration and spectral attributes appears to be more complex. These findings are discussed for vowels preceding postvocalic voiceless and voiced consonants.

5pSC23. Isolating the critical segment of $\mathrm{AE} / \mathrm{r} /$ and $/ /$ to enhance non-native perception. Rieko Kubo, John S. Pruitt, and Reiko AkahaneYamada (ATR Human Information Processing Res. Labs., 2-2 Hikaridai, Seika-cho, Soraku-gun, Kyoto, 619-02 Japan, rkubo@hip.atr.co.jp)

Brief synthetic stop-consonant+vowel syllables as short as $20 \mathrm{~ms}$ are reliably identified for place of articulation. Further, removal of the steadystate vowel in synthetic stop-consonant+vowel series improves discrimination performance, but does not affect identification. Subsequently, vowel truncation of consonant-vowel syllables has been used to improve native English speaker's perception of Hindi dental and retroflex stopconsonants. Whether such truncation techniques are effective for native speakers of Japanese on the AE liquids /r/ and /l/ was examined. The /r/ or /1/ segment from 50 minimal word pairs, produced in five phonemic positions, was isolated by truncating the surrounding phonemic environment. Six durations of the segment were created: 60, 100, 140, 180, $220 \mathrm{~ms}$, and full-length (unedited productions). Native Japanese speakers were asked to identify the target phoneme for each segment, which was presented in a random sequence but blocked by speaker and segment duration. Segment duration significantly affected performance. Interestingly, while longer segments were perceived better than full-length stimuli, shorter segments were not perceived as well. Identification training with these stimuli confirmed the effectiveness of such manipulations. The implications of these findings are discussed with regard to theories of cross-language speech perception and methods of training non-native contrasts.

5pSC24. English vowel production by native speakers of Beijing Mandarin. Xinchun Wang and Murray J. Munro (Dept. of Linguist., Simon Fraser Univ., Burnaby, BC V5A 1S6, Canada, jxwang@sfu.ca)

Ten English vowels produced in a controlled elicitation paradigm by 15 Beijing Mandarin speakers and 15 native English speakers were randomly presented to trained English listeners who identified them in a forced-choice listening task. Although the non-native productions of the vowels with Mandarin counterparts were generally as intelligible as those of the native English speakers, the remaining vowels were produced with varying degrees of intelligibility. The results suggested a strong influence of the $L 1$ vowel system on the production of $L 2$ vowels. In particular, acoustic analyses revealed that the acccented English vowels tended to be "pulled" toward their Mandarin counterparts so that they fell between the English and Mandarin vowels in an $F 1-F 2$ space. Spectral overlap in the tense-lax pairs accounted for some intelligibility problems, while the dense distribution of second-language $(L 2)$ vowels in the area of the vowel space where the $L 1$ vowel system is less crowded apparently increased the level of difficulty for the $L 2$ learners in producing some vowels. The results are discussed in terms of error prediction models for $L 2$ speech learning.
5pSC25. The effect of immersion on second language productions: The acquisition of American English / $\mathbf{r} /$ and /// by Japanese children. Jesica C. Downs-Pruitt, Reiko Akahane-Yamada (ATR-HIP Labs, 2-2 Hikaridai, Seika-cho, Soraku-gun, Kyoto, 619-02 Japan, jcpruitt@hip.atr.co.jp), Reiko Mazuka (Duke Univ., Durham, NC 27708), and Akiko Hayashi (Tokyo Gakugei Univ., 4-1-1, Nukui-Kita, Koganei-Shi, 184 Japan)

Many studies have shown that the American English (AE) /r/ and /// are difficult for adult native speakers of Japanese to perceive and produce, sometimes even after substantial experience and/or intense training. In fact, there are many reports of first language $(L 1)$ effects on second language (L2) learning. Common lore promotes immersion as the best method of second language learning, particularly for overcoming $L 1$ biases. The current study examined the effect of English-language immersion on Japanese children's productions of $\mathrm{AE} / \mathrm{r} /$ and $/ 1 /$. We studied productions from children in three age groups, 4-, 6-, and 8-year-olds. Native Japanese children, who had been immersed in an English-language environment, nonimmersed Japanese children, and native English children produced English-language words and nonwords which included /r/, /l/, and $/ \mathrm{w} /$ in three positions. The productions were evaluated by categorizing them into one of seven categories, which included a "correct" and "distorted" category for each intended gesture, as well as an "other" category. Significant differences in intelligibility were found dependent upon the age and language-experience group. Furthermore, the children in the three language-experience groups showed different substitution patterns. The results are discussed in the context of recent theories of $L 1$ development and $L 2$ learning.

5pSC26. Modification of $L 2$ vowel production by perception training as evaluated by acoustic analysis and native speakers. Reiko Akahane-Yamada (ATR HIP Labs., Seika, Soraku, Kyoto, 619-02 Japan, yamada@hip.atr.co.jp), Winifred Strange (Univ. of South Florida, Tampa, FL 33620-8150), Jesica C. Downs-Pruitt (ATR HIP Labs., Seika, Soraku, Kyoto, 619-02 Japan), and Yasushi Masuda (Grad. School of Information Sci. NAIST, 8916-5 Takayama, Ikoma, Nara, 630-01 Japan / ATR HIP Labs.)

Recent cross-language studies have shown that there is a link between speech perception and speech production when learning second language (L2) consonants. This paper reports additional evidence for such a link from a study which examines whether identification training of $L 2$ vowels improves the trainees' production ability. Twenty native speakers of Japanese served as subjects. Half of the subjects received a pretest, identification training on English /a, æ, $N /$ and a post-test. For the other half of the subjects, only the pretest and post-test were administered. In addition to the perception test, productions of the English vowels were recorded in the pre- and post-test. These productions were later examined in two ways; acoustical analysis and evaluation by native English speakers. Identification ability significantly improved from pretest to post-test for the trained group, but not for the untrained group as reported by Strange and Akahane-Yamada [J. Acoust. Soc. Am. 102, 3137 (1997)]. More importantly, production ability showed a significant improvement from pretest to post-test for the trained group, suggesting that there is a link between vowel perception and vowel production. The results are discussed in the context of the theories of $L 2$ learning.

5pSC27. Categorial discrimination of English and Japanese vowels and consonants by native Japanese and English subjects. Susan G. Guion, James E. Flege (Dept. of Rehab. Sci., VH503, Univ. of Alabama, Birmingham, AL 35294, guionsg@uab.edu), Reiko Akahane-Yamada, and Jesica C. Downs-Pruitt (ATR, Kyoto, 619-02 Japan)

Categorial discrimination tests (CDTs) with equal opportunities for "hits" and "false alarms"' assessed native Japanese and English subjects' perception of English and Japanese segmental contrasts. The stimuli consisted of vowels and consonants spoken at two rates by five native speakers each of English and Japanese. The listeners chose the odd item out in 
triads, if there was one, or responded "none" if they heard three physically different tokens of a single vowel or consonant. A' scores were computed for each contrast. The subjects were ten native English (NE) controls, ten "experienced" bilinguals (Japanese speakers who had spent several years in the United States), and ten "inexperienced" bilinguals (Japanese speakers who had never lived outside Japan, but had studied English in school). Analysis of the CDT results will help determine (1) if the subjects differ in their ability to discriminate consonants and vowels, (2) if perceptual sensitivity to vowel and/or consonant contrasts is affected by the speech rate, and (3) if the experienced bilinguals more closely resemble the NE controls than the inexperienced bilinguals do. [Work supported by NIH.]

5pSC28. Effects of listener training on intelligibility of Chineseaccented English. Catherine L. Rogers (Dept. of Speech and Hearing Sci., Ohio State Univ., 110 Pressey Hall, 1070 Carmack Rd., Columbus, OH 43210, rogers.140@osu.edu)

Previous research has demonstrated significant improvements in the intelligibility of synthetic speech following listener training [E. Schwab et al., Human Factors 27, 395-408 (1985)]. In the present study, the effect of listener training on second-language speech intelligibility was investigated. Chinese-accented words and sentences were presented to four groups of native English listeners-one training and one control group for each of two speakers. On word trials, listeners were instructed to select the word presented from two minimal-pair alternatives; on sentence trials, listeners were instructed to write down what they heard. Listener responses were scored as percent of intended words correctly identified. The two training groups participated in three $1-\mathrm{h}$ training sessions. Auditory and visual feedback was provided to listeners during the training sessions only. Performance of the training and control groups was compared across pre- and posttraining test sessions. Improvement in minimal-pair word intelligibility following training was found across speakers for the training group, relative to the control group; a similar but smaller improvement in sentence intelligibility was found. Word results will be discussed in terms of improvement in discriminability following training for the twelve phonemic contrasts tested. [Work supported in part by NIH-NIDCD Grant No. 2R44DC02213.]

5pSC29. An instance-based model of Japanese speech recognition by native and non-native listeners. Kiyoko Yoneyama and Keith Johnson (Dept. of Linguist., Ohio State Univ., 222 Oxley Hall, 1712 Neil Ave., Columbus, OH 43210)

Japanese has a short and long segment contrast (oji-san versus ojiisan, kita versus kitta, and kana versus kanna). Previous research has shown that this durational property is one of the characteristics of moras in Japanese. It has also been reported that Japanese learners generally have difficulty in acquiring this contrast, but after extensive language experience with Japanese, they do learn the contrast. This suggests that the contrasts and similarities are based on remembered instances of linguistic objects in various environments during a lifetime. This paper investigates whether an instance-based approach of speech recognition can replicate performance by native listeners and second language learners of Japanese. An instance-based model was trained with naturally produced utterances of koma spin and komma comma and then tested on a 24-step nasal duration continuum from koma to komma [T. Uchida, doctoral dissertation, Nagoya University (1996)]. The identification function produced by the model was very similar to identification responses produced by native Japanese listeners. Additional simulations trained with utterances produced by English native speakers (e.g., coma, comma) will be reported. These simulations will address the role of linguistic experience in second language acquisition. [Supported by NIDCD R29-016435-06.]
5pSC30. Training American listeners to perceive and produce Mandarin tones. Yue Wang, Allard Jongman, and Joan A. Sereno (Dept. of Linguist. and Modern Lang., Cornell Univ., Ithaca, NY 14853, yw36@cornell.edu)

Auditory training of non-native speech contrasts is based on the assumption that the perceptual system of adults can be modified. Previous research has shown substantial improvements in the identification of segmental distinctions which are absent in the listeners' native language after a simple phonetic laboratory training procedure. However, it is not known whether such a training procedure is applicable to the acquisition of nonnative suprasegmental contrasts. In the present study, native speakers of English are trained to perceive Mandarin lexical tones, using the highvariability procedure shown to be efficient in previous studies concerning the generalization and long-term retention of the training effects. In 20 sessions lasting four weeks, ten American learners of Chinese are trained to identify the four tones in natural words occurring in different phonetic contexts, and spoken by different talkers. The results of the pre- and posttests are compared to determine if suprasegmental contrasts can be obtained and improved by training, if the contrasts obtained can be generalized to novel words and talkers, if contrasts can be maintained long after training, and if learning gained perceptually can be transferred to production. The results will be discussed in terms of nonnative perceptual modifications at the suprasegmental level.

5pSC31. A CALL system for teaching the duration and phone quality of Japanese tokushuhaku. Goh Kawai and Keikichi Hirose (Univ. of Tokyo, Dept. of Information and Commun. Eng., 7-3-1 Hongo, Bunkyo-ku, Tokyo, 113 Japan, goh@kawai.com)

This paper describes a CALL (computer-aided language learning) system that teaches the pronunciation of Japanese tokushuhaku (long vowels, the mora nasal, and mora obstruents) to beginning learners of Japanese as a second language. The system detects mispronounced tokushuhaku durations and quality, including, for instance, shortened or lengthened tokushuhaku, dentalized mora nasals, diphthongized long vowels, and aspirated plosives. Learners are prompted by the system to read tokushuhaku minimal pairs aloud. Tokushuhaku durations are measured using speech recognition and compared with distributions of native speakers. Mistakes in phone quality are detected using a speech recognizer incorporating bilingual monophone models of both the learner's native and target languages. HMMs for the two languages are trained separately on languagedependent speech data, but are bundled together during recognition so that the closest phone recognized indicates the non-nativeness of the utterance should the recognized phone not be Japanese. Knowing the phonetics and phonology of the learner's native language can identify non-native articulatory gestures that result in Japanese pronunciation errors, thus allowing precise corrective feedback to the learner. The paper includes an overview of the system, its reliability, validity, and effectiveness in the foreigen language classroom.

5pSC32. The organization of articulator gestures: A comparison of Swedish, Bulgarian, and Greenlandic. Sidney A. J. Wood (Dept. of Linguist., Univ. of Lund, Helgonabacken 12, S-22362 Lund, Sweden, sidney.wood@ling.lu.se)

This paper reports speech articulator gestures in Swedish, Bulgarian, and Greenlandic to study universal and language-specific components in articulation. This work is based on analysis of movements of individual articulators from $\mathrm{x}$-ray motion films of speech, and continues from previous reports from this study by Wood [J. Phon. 7, 25-43 (1979); 19, 281-292 (1991); Proc. 3rd Conf. I.C.P.L.A., 191-200, Helsinki (1994); Proc. 13th I.C.Ph.S. Vol. 1, 392-395, Stockholm (1995); J. Phon. 24, 139-164 (1996); Proc. 4th Speech Prod. Sem., 61-64, Grenoble (1996); Speech Commun., (in press)], and by Wood and Pettersson [Folia Ling. 22, 239-262 (1988)]. Common principles concerned utilization and integration of articulator gestures (articulator gestures executed in approach, hold, and withdrawal phases, four tongue body gestures used, all gestures 
available for vowels and consonants, gesture conflicts resolved by gesture queuing, coarticulation and assimilations implemented by coproduction). Language-specific principles concerned implementation of assimilations like palatalization of alveolar stops in Bulgarian and uvularization of vowels in Greenlandic. One assimilation, palatalization of velar consonants, is common to all three languages. A model of gesture programming based on these results is proposed.

5pSC33. The relationship between non-native phoneme perception and the MMN. Janet W. Stack and Susan D. Dalebout (Commun. Disord. Prog., Univ. of Virginia, 2205 Fontaine Ave., Charlottesville, VA 22903 jws8n@virginia.edu)

The mismatch negativity (MMN) auditory-evoked potential reflects the detection of minimal stimulus differences at a preattentive level. It has been reported that the MMN reflects acoustic, rather than phonetic processing of speech stimuli. The purpose of the present study was to examine the relationship between discrimination at a neurophysiologic level, as reflected by the MMN, and the behavioral ability of native speakers of American English to discriminate and label pairs of Hindi stop consonants that are contrasted by a place of articulation feature that is not phonemic in English. These pairs were the voiceless unaspirated Hindi stop consonants, dental and retroflex /t/, and the voiced unaspirated Hindi stops, dental and retroflex /d/. The present study addressed two questions: (1) whether the MMN response would be evoked by acoustic differences resulting from differences in place of articulation, even though behavioral discrimination ability was poor; and (2) whether there would be a difference in the presence/absence or other parameters (latency, duration, amplitude, and area) of the MMN response depending on which voicing contrast was presented.

5pSC34. Acquisition of language-specific word-initial unvoiced stops: VOT, intensity, and spectral shape in American English and Swedish. Eugene H. Buder (Univ. of Memphis, Memphis, TN 38105, ehbuder@memphis.edu) and Carol Stoel-Gammon (Univ. of Washington, Seattle, WA 98105-6246)

By 30 months of age, children learning American English (AE) or Swedish (S) have begun to acquire language specific aspects of wordinitial /t/s [Stoel-Gammon et al., Phonetica 51, 146-158 (1994)]. The coronal stop is ostensibly alveolar in $\mathrm{AE}$ and dental in $\mathrm{S}$, suggesting a place mechanism for the distinctive acoustic measures obtained from both adults and toddlers from these languages (specifically burst frequency shape as measured by spectral mean and standard deviation, and burst intensity as calibrated to following vowel). However, these measures, as well as voice onset time, are also distinctive in the same directions for other unvoiced stop consonants in adults and children, suggesting a system-wide mechanism for language-specific behaviors in this domain. Current results on word-initial /p/s, /t/s, and / $/ \mathrm{s}$ from both 24-month- and 30-month-old children are interpreted as evidence for early acquisition of specific allophonic phonetic aspects of consonants. [Work supported by NICHD.]

5pSC35. The effects of postvocalic voicing on the duration of high front vowels in Swedish and American English: Developmental data. Carol Stoel-Gammon (Univ. of Washington, Seattle, WA 98105-6246) and Eugene H. Buder (Univ. of Memphis, Memphis, TN 38105)

In most languages of the world, a vowel preceding a voiced obstruent is longer than the same vowel preceding a voiceless obstruent. Although the effect of postvocalic voicing on vowel duration is often considered to be phonetically driven, the extent of influence differs considerably across languages. In English, for example, vowels preceding voiced obstruents are nearly twice as long as those preceding voiceless obstruents, whereas in Swedish, the influence is minimal, perhaps because vowel length is phonemically contrastive in this language. The present study examines acquisition data from Swedish and American children, aged 24 and 30 months, to determine developmental patterns of vowel duration associated with context-sensitive voicing. It was hypothesized that the 24-month-old children from both language backgrounds would exhibit the phonetically based tendency for vowels to be significantly longer in the voiced context, but that at 30 months of age, the effects of final consonant voicing would be much stronger in English than in Swedish. Durational measures of high front vowels (tense/long /i/ and its short/lax counterpart) supported the proposed hypothesis. [Work supported by NICHD.]

5pSC36. Perceptual assimilation of Hindi dental and retroflex stopconsonants by native English and Japanese speakers. John S. Pruitt, Reiko Akahane-Yamada (ATR Human Information Processing Res. Labs., 2-2 Hikaridai, Seika-cho, Soraku-gun, Kyoto, 619-02 Japan, jspruitt@hip.atr.co.jp), and Winifred Strange (Univ. of South Florida, Tampa, FL 33620)

Previous research has shown that English speakers have great difficulty distinguishing the dental and retroflex stop-consonants of the Hindi language. However, native Japanese speakers have somewhat less difficulty perceiving this contrast even though it is not employed in the Japanese language. Best and colleagues have attributed similar differences in the perception of non-native speech sounds to differences in the assimilation of such sounds to native-language speech categories [Best, McRoberts, and Sithole, J. Exp. Psych: Human Percept. Perform. 14 (1988)]. According to Best's Perceptual Assimilation Model (PAM), four patterns of assimilation have been described which are purportedly predictive of perceptual difficulty. To determine if this model could be applied to the present case, native speakers of English and Japanese transcribed multiple instances of the Hindi consonants in different voicing/manner classes, produced with different vowels. Marked differences were found between the responses of the two language groups which were partially dependent upon the voicing/manner class of the contrast. Interestingly, an assimilation pattern emerged which was different from those discussed in PAM. Implications of these findings for PAM and for perceptual training of non-native speech sounds will be discussed. [Work supported in part by NIDCD.]

5pSC37. Comparison of American English vowel production and identification by native speakers of Luso-Portuguese. Christina F. Famoso, Patricia N. Schwartz (Dept. of Speech, Commun. Sci., and Theatre, St. John's Univ., Jamaica, NY 11439), and Adelia DaSilva (Blythedale Children's Hospital, Valhalla, NY)

Learning a new language requires mastering the phonetic as well as lexical and syntactical aspects of the new language. As part of a larger study of second-language learning, the identification of American English vowels by speakers of Luso-Portuguese and the identification of those speakers' productions by native speakers of American English were explored. Four adult bilingual Luso-Portuguese/English speakers (two women and two men) participated in this study. The subjects performed two tasks. First, they identified ten English words (ten repetitions of each, presented in random order, of: heed, hid, head, had, hod, hawed, hood, who'd, hud, heard) produced by a native speaker of American English. Then recordings were made of the subjects as they produced four repetitions each, in random order, of these ten English words. These recordings were digitized using SoundDesigner II software with an audiomedia board on a Macintosh Quadra 800 computer. Listening tapes will be prepared, using SoundDesigner II software, for identification of the Luso-Portuguese speakers' vowels by native American English speakers. The results of the 
identification of the Luso-Portuguese speakers' vowels will be compared with the identification of American English vowels by the native LusoPortuguese speakers. [Work supported by St. John's University.]

5pSC38. Acoustic analysis of American English vowels by native speakers of Luso-Portuguese. Patricia N. Schwartz, Christina F. Famoso (Dept. of Speech, Commun. Sci., and Theatre, St. John's Univ., Jamaica, NY 11439), and Adelia DaSilva (Blythedale Children's Hospital, Valhalla, NY)

The ability of individuals to learn a new language requires mastering the phonetic as well as lexical and syntactical aspects of the new language. As part of a larger study of second-language learning, the acoustical characteristics of American English vowel production by native speakers of Luso-Portuguese were explored, and these characteristics were compared with the identification of these speakers' English vowels by native speakers of American English. Four adult bilingual Luso-Portuguese/English speakers (two women and two men) participated in this study. Recordings were made of the subjects as they produced four repetitions each, in random order, of 10 English words (heed, hid, head, had, hod, hawed, hood, who'd, hud, heard). The recordings were digitized using SoundDesigner II software with an audiomedia board; formant frequency analyses will be carried out using Signalyze 2.0 software implemented on a Macintosh Quadra 800 computer. The acoustical analyses will be examined in light of the identification of the Luso-Portuguese speakers' English vowels by native speakers of American English (reported in another paper at this meeting). [Work supported by St. John's University.]

5pSC39. Comparison of American English vowel production and identification by native speakers of Russian. Fredericka Bell-Berti (Dept. of Speech, Commun. Sci., and Theatre, St. John's Univ., Jamaica, NY 11439), Lisa Jayne Romano, and Eugenia Lorin (St. John's Univ., Jamaica, NY 11439)

Learning a new language requires mastering the phonetic as well as lexical and syntactical aspects of the new language. As part of a larger study of second-language learning, the identification of American English vowels by speakers of Russian and the identification of those speakers' productions by native speakers of American English were explored. Four adult bilingual Russian/English speakers (two women and two men) participated in this study. The subjects performed two tasks. First, they identified ten English words (ten repetitions of each, presented in random order, of: heed, hid, head, had, hod, hawed, hood, who'd, hud, heard) produced by a native speaker of American English. Then recordings were made of the subjects as they produced four repetitions each, in random order, of these ten English words. These recordings were digitized using SoundDesigner II software with an audiomedia board on a Macintosh Quadra 800 computer. Listening tapes will be prepared, using SoundDesigner II software, for identification of the Russian speakers' vowels by native American English speakers. The results of the identification of the Russian speaker's vowels will be compared with the identification of American English vowels by the native Russian speakers. [Work supported by St. John's University.]

5pSC40. Acoustic analysis of American English vowels by native speakers of Russian. Lisa Jayne Romano (Dept. of Speech, Commun. Sci., and Theatre, St. John's Univ., Jamaica, NY 11439), Fredericka Bell-Berti (St. John's Univ., Jamaica, NY 11439), and Eugenia Lorin (St. John's Univ., Jamaica, NY 11439)

The ability of individuals to learn a new language requires mastering the phonetic as well as lexical and syntactical aspects of the new language. As part of a larger study of second-language learning, the acoustical char- acteristics of American English vowel production by native speakers of Russian were explored and these characteristics were compared with the identification of these speakers' English vowels by native speakers of American English. Four adult bilingual Russian/English speakers (two women and two men) participated in this study. Recordings were made of the subjects as they produced four repetitions each, in random order, of ten English words (heed, hid, head, had, hod, hawed, hood, who'd, hud, heard). The recordings were digitized using SoundDesigner II software with an audiomedia board; formant frequency analyses will be carried out using Signayze 2.0 software implemented on a Macintosh Quadra 800 computer. The acoustical analyses will be examined in light of the identification of the Russian speakers' English vowels by native speakers of American English (reported in another paper at this meeting). [Work supported by St. John's University.]

5pSC41. Is allophonic variation in Japanese / $/$ a factor in Japanese listeners' difficulty in perceiving English $/ \mathrm{I}-\mathrm{I} /$ ? Mieko Ueno, Patrice $\mathrm{S}$. Beddor (Prog. in Linguist., Univ. of Michigan, Ann Arbor, MI 48109, beddor@umich.edu), and Reiko Akayane-Yamada (ATR Human Information Processing Res. Labs., Kyoto, Japan)

Japanese listeners' difficulty in perceiving the English liquid (/I-1/) contrast has been attributed to various phonological and phonetic factors. To explore allophonic variation in the Japansese flap as a contributing factor, American English listeners' perception of Japanese / $\mathrm{r} /$ was investigated in a range of phonetic contexts. Stimuli were 152 Japanese words containing / $\mathrm{f} /$ in all phonotactically possible contexts produced by two native Japanese speakers. Three stimulus randomizations were presented to 12 American English listeners for identification as "l," "r," " $D$," (=flap), “d," “t," “'no sound," or "'other sound." Results showed strong effects of syllable structure: Japanese / $\mathrm{r} /$ sounded more stoplike (" $\mathrm{D}$," " $\mathrm{d}$, ," " $\mathrm{t}$ "' responses) in consonantal contexts and more liquidlike ("r," "l'" responses) in vocalic contexts. However, liquid responses were more likely to be " $r$ " intervocalically, compared with " 1 " in initial position. Vowel quality also had a strong effect: Japanese / $\mathrm{f} /$ elicited more stop responses in high vowel ([i u $]$ ) contexts and more liquid responses in lower vowel ([a o $]$ ) contexts. Acoustic measures of the stimuli were generally consistent with the perceptual patterns. The perceptual and acoustic findings are compared with the results of recent training studies [AkayaneYamada, Proc. 3rd Joint Meeting of ASA-ASJ, 953-958 (1996)] with Japanese listeners on English / $\mathrm{I}-1 /$.

5pSC42. The perception of place of articulation in three coronal nasal contrasts. James D. Harnsberger (Prog. in Linguist., Univ. of Michigan, 1076 Frieze Bldg., Ann Arbor, MI 48109, jharns@umich.edu)

Place of articulation contrasts which undergo perceptual assimilation are often difficult to discriminate if absent in a listener's native language, as demonstrated by American English (AE) listeners with Hindi dentalretroflex stops [Polka (1991)] and Salish velar-uvular ejectives [Werker and Tees (1984)]. To see if this pattern generalizes to other manners of articulation, nasal contrasts (dental-alveolar, dental-retroflex, alveolarretroflex) from three languages (Malayalam, Marathi, and Oriya), varying in talker, syllabic position, and vowel context, were presented to speakers of Malayalam, Marathi, and Oriya (control groups), and Bengali, AE, Tamil, and Punjabi in two tests: AXB discrimination, and identification with goodness ratings. AE and Bengali were chosen because both possess 
a single relevant perceptual category for these stimuli, an alveolar nasal. Tamil and Punjabi were selected for their two nasal categories, dental and retroflex. Of the contrasts tested, Malayalam dental-alveolar was the most challenging, with all non-native groups performing at-chance on the AXB test. The discriminability of the other contrasts proved to be highly dependent on the particular talker, syllable position, or vowel context, with performance ranging from at-chance to $98.4 \%$. An acoustic analysis of the stimuli will also be provided.

5pSC43. Vowel perception by formant variation. Byunggon Yang (English Dept., Dongeui Univ., Kayadong Pusanjingu, Pusan 614-714, Korea)

The acoustic parameters of six Korean vowels produced by a healthy male subject with normal hearing were analyzed to synthesize the six vowels by a formant synthesis method. The $f_{0}$, amplitude envelopes, and the first six formant values were elaborately matched to the those of the original vowels. Then, $F_{1}$ and $F_{2}$ values of the synthesis file were modified by $50 \mathrm{~Hz}$ over and under the original values but not interfering with adjacent formants. $F_{3}$ varied $500 \mathrm{~Hz}$ over and under the original values by $100 \mathrm{~Hz}$ to obtain 270 stimuli. Twenty male and female subjects in a quiet room listened to the original vowel followed by the corresponding synthesized one $0.5 \mathrm{~s}$ later and judged whether they sounded qualitatively "similar" or "different." From the experiment, it was found that male subjects responded "similar" within the range of variance by an average of $163 \mathrm{~Hz}$ for $F_{1}, 415 \mathrm{~Hz}$ for $F_{2}$; and $843 \mathrm{~Hz}$ for $F_{3}$. Female subjects showed $40-\mathrm{Hz}$ higher range than males. The ranges supported the nonlinearity of the human auditory scale in the speech sound. This suggests that the validity of exact formant frequency measurement varies according to frequency levels.

5pSC44. Cross-linguistic evidence for the acquisition of accent by the onset of word use. Rory A. DePaolis, Marilyn M. Vihman (Dept. of Psych., Univ. of Wales, Bangor, Bangor LL57 2DG, Gwynedd, UK, r.depaolis@bangor.ac.uk), and Leonard P. Lefkovitch (Carleton Univ., Ottawa, Canada)

An investigation of the acoustic correlates of stress or accent in infant vocalizations was undertaken using five English- and five French-learning participants at two developmental points, the onset of word use (10-13 months) and late in the single-word period (14-19 months). A data base of 555 disyllabic utterances (words and babble) was digitized from naturalistic recordings and quantified acoustically by indices of duration, amplitude, and fundamental frequency $\left(F_{0}\right)$. Cluster analysis as well as the results of perceptual judgments (i.e., both a bottom-up and a top-down approach) revealed a developmental trend toward the adult prosodic system. In addition, acoustic comparisons of French and American infants at both developmental points revealed differences in their use of amplitude, $F_{0}$, and duration to accent a syllable. The differences were traceable to the adult patterns for the respective adult languages. These results suggest that infants' perceptual attunement to prosody in the prelinguistic period manifests itself in the beginnings of adultlike production as early as the onset of word use. [Work supported in part by the UK Economic and Social Research Council.]

FRIDAY AFTERNOON, 26 JUNE 1998

GRAND BALLROOM B (S), 1:00 TO 5:00 P.M.

\title{
Session 5pUW
}

\section{Underwater Acoustics: Bistatic Scattering From the Seabed and Surface: Models and Measurements II}

\author{
Paul C. Hines, Chair \\ Defence Research Establishment Atlantic, P.O. Box 1012, Dartmouth, NS B2Y 3Z7, Canada
}

Chair's Introduction-1:00

Invited Papers

1:05

5pUW1. Implications of a bistatic treatment for the second echo from a normal incidence sonar. Gary J. Heald (Defence Evaluation and Res. Agency, Weymouth, Dorset, England) and Nicholas G. Pace (Univ. of Bath, Claverton Down, Bath, England)

Echo sounders have traditionally been used for the measurement of water depth but recent developments have shown how the information contained in the acoustic backscatter, related to the sediment type, may be extracted. As echo sounders operate at normal incidence it is possible to use the first and second backscatter from the seabed for sediment discrimination. This study has investigated why additional information may be contained in the second backscatter. It is shown that the geometry for the second backscatter must be treated as an on-axis bistatic configuration in which the source is effectively 3 water depths above the seabed. The resulting scattering equations are used to show how the range dependence of the scattering places the receiver in the near field for the second echo. The importance of the sensor geometry and the processing of the received signals are also discussed. As a result, it is shown that the combination of information contained in the first and second backscatter may be used to descriminate between different sediments. The result from tank experiments using a $200-\mathrm{kHz}$ transducer over different sediments has allowed comparison with the theory and shows the effect of different water surface roughness on the second backscatter.

\section{$1: 25$}

5pUW2. In-plane bistatic calculations of sub-bottom volume reverberation in shallow water. Dale D. Ellis and Paul C. Hines (Defence Res. Establishment Atlantic, P.O. Box 1012, Dartmouth, NS B2Y 3Z7, Canada)

A shallow-water reverberation model based on normal modes and ray-mode analogies has been previously developed [J. Acoust. Soc. Am. 97, 2804-2814 (1995)]. It was first developed for boundary scattering and recently extended to handle volume reverberation in both the water and sub-bottom. The volume scattering is formulated in terms of an empirical plane-wave scattering function, which depends on depth but which can also depend on the incident and scattered angles. Our first approximation for the volume reverberation 
is to choose the scattering to be isotropic, but to get a better approximation based on physical parameters, the in-plane bistatic scattering function of Hines is used [J. Acoust. Soc. Am. 99, 836-844 (1996)]. In this paper, calculations of shallow-water reverberation are made with the Hines scattering function used as input to the normal-mode model. Results are compared with reverberation assuming isotropic scattering in the bottom, as well as with reverberation due to bottom interface scattering using Lambert's rule.

\section{Contributed Papers}

\section{$1: 45$}

5pUW3. A review of the SSA for rough surface scattering. Shira L. Broschat (School of Elec. Eng. and Comput. Sci., Washington State Univ., P.O. Box 642752, Pullman, WA 99164-2752) and Eric I. Thorsos (Univ. of Washington, Seattle, WA 98105)

The small slope approximation (SSA) for scattering from rough surfaces was introduced by Voronovich in the mid-1980s [A. G. Voronovich, Sov. Phys. JETP 62, 65-70 (1985)]. Numerical studies have shown that the SSA gives excellent results over a broad range of scattering angles. However, in the high-frequency limit the second-order (in the field) SSA scattering amplitude reduces to the geometrical optics result and, thus, to a single scattering model limited to local interactions with the surface. In this paper, small slope theory will be reviewed briefly, expressions for the bistatic scattering cross section will be presented, and numerical results will be shown for a number of different problems and several different roughness spectra. In addition, in an effort to extend the nonlocal scattering effects, Voronovich modified the SSA. He applied the same approach underlying the SSA, but he began by iterating the integral equation of the second kind [A. G. Voronovich, Waves Random Media 6, 151-167 (1996)] which led to the nonlocal small slope approximation (NLSSA). While the main focus of this talk will be the SSA, the NLSSA will be compared with the SSA for selected results. [Work supported by ONR.]

\section{$1: 57$}

5pUW4. The lowest-order small slope approximation for rough surface scattering. Eric I. Thorsos (Appl. Phys. Lab., College of Ocean and Fishery Sci., Univ. of Washington, Seattle, WA 98105) and Shira L. Broschat (Washington State Univ., P.O. Box 642752, Pullman, WA 99164-2752)

The small slope approximation (SSA) [A. G. Voronovich, Sov. Phys. JETP 62, 65-70 (1985)] for scattering from rough surfaces leads to a systematic series and improved accuracy is obtained by using higher terms in the series. Numerical implementation of the lowest-order SSA for the scattering cross section has the same level of effort required as for the Kirchhoff approximation (a single integration for one-dimensional surfaces, a double integral for two-dimensional surfaces), while higher orders require higher-order integrations. Thus the accuracy of the lowest-order SSA is of considerable practical interest. In this paper, the accuracy of lowest-order SSA will be illustrated for surfaces with power-law roughness spectra through comparison with exact numerical results. Both Dirichlet and two-fluid boundary conditions will be considered. For typical power-law spectra, the accuracy of the lowest-order SSA is generally quite good. [Work supported by ONR.]

\section{2:09}

5pUW5. The Kirchhoff approximation: A new iterative solution. Suzanne T. McDaniel (4035 Luxury Ln., Bremerton, WA 98311)

A new iterative solution to the problem of sea surface scattering is obtained which has the Kirchhoff approximation as its leading term. This solution is obtained by transforming an integral equation of the first kind for the surface potential to one of the second kind, and applying the method of successive approximations. The advantages of this method are that the conditions under which this solution converges may be readily established and the solution may be iterated to sufficiently high order in the interaction to examine the rate at which it converges. A numerical example of scattering from an echelette grating is considered, and the regime of convergence established. It is found that the new iterative solution converges only for such surfaces on which $k \sigma \sigma^{\prime}<\pi$ where $k$ is the wave number of the scattered radiation and $\sigma$ and $\sigma^{\prime}$ are, respectively, the rms surface height and slope. This finding raises doubts concerning the regime in which the Kirchhoff approximation is traditionally deemed applicable. Finally, numerical studies are presented to illustrate the convergence of the iterative solution.

\section{2:21}

5pUW6. Statistical characteristics of bistatic sea surface scatter. Peter D. Neumann (Planning Systems, Inc., 7923 Jones Branch Dr., McLean, VA 22102) and R. Lee Culver (Penn State Univ., University Park, PA 16804)

The characterization of reverberation, from the surface, the bottom, and/or the volume, is important in advancing the understanding of the mechanisms involved in underwater sound scattering. Numerous experiments and much theoretical work have been done in the area of monostatic reverberation, but the area of bistatic reverberation has been much less researched. This certainly is a result of the much more complex geometries involved in bistatic reverberation. This work studies the statistics of bistatic sea surface reverberation data from the FLIP experiment conducted in January of 1992. The data were verified as homogeneous and normally distributed using the Kolmogorov-Smirnov two-sample test with a confidence interval, $\alpha$, equal to 0.1 . Using a technique by which ensembles from different times were combined, meaningful deviations from a normal distribution were observed at the highest wind speed of 7.2 $\mathrm{m} / \mathrm{s}$. The bistatic surface scattering strengths were calculated from the data and compared with the predictions of a theory developed by S. T. McDaniel for the prediction of bistatic surface scattering strengths. The comparison showed good agreement with a rms deviation of about $3 \mathrm{~dB}$. [Work supported by ONR.]

\section{2:33}

5pUW7. Time dependence in forward scattering: Experimental results and model comparisons derived from sea surface and seabed bistatic cross sections. Peter H. Dahl and Kevin L. Williams (Appl. Phys. Lab., Univ. of Washington, Seattle, WA 98195)

In shallow, littoral regions the acoustic transmission channel can often be governed by the process of forward scattering from both the sea surface and seabed. In this paper, measurements of the intensity time dependence of high-frequency $\mathrm{O}(10) \mathrm{kHz}$ sound that has been forward scattered from both the sea surface and seabed are discussed along with simulated time series for this process. The data are from an experiment conducted near Key West, Florida in waters 25-m deep, for which the seabed was characterized by a carbonate sand-silt-clay mixture. The simulated time series are derived from the bistatic cross sections for sea surface and seabed by convolving the bistatic scattering impulse response with the waveform emitted from the transmitter. For the seabed in situ estimates of sediment properties were used as inputs to a model described in Williams and Jackson [J. Acoust. Soc. Am. (submitted)]. For the sea surface, the necessary representation of the two-dimensional surface wave correlation function was derived from estimates of the directional wave spectrum. The latter were measured with a directional wave buoy that operated in the immediate vicinity of the acoustic measurements. [Work supported by ONR Code 321OA.] 
ring projectors and two hydrophones was used to measure monostatic and bistatic scatter in $100 \mathrm{~m}$ of water on the Scotian Shelf. A series of linear

5pUW8. Stacking and averaging techniques for bottom echo characterization. Daniel D. Sternlicht and Christian P. deMoustier (Marine Physical Lab., Scripps Inst. of Oceanogr., 9500 Gilman Dr., La Jolla, CA 92093-0205)

Bottom-looking sonars operating at frequencies greater than $30 \mathrm{kHz}$ transmit acoustic signals which penetrate at most a few meters into seafloor sediments, making them well suited to characterize the water/ sediment interface. Acoustic wavelengths at these frequencies are small compared to the rms relief of the interface, resulting in bottom echoes dominated by incoherent energy and varying significantly in amplitude and shape as the sonar translates longitudinally above the interface. Because of this variability, echoes must be treated stochastically and determining the average shape of the bottom echo involves careful selection of stacking and averaging algorithms. Comparisons of a geoacoustic temporal model with 33- and $93-\mathrm{kHz}$ backscatter echoes from sand and silt substrates reveal that a representative echo shape is obtained by averaging echoes along-track over a distance roughly equivalent to the 6-dB footprint diameter of the sonar beam pattern. To be meaningful, such averaging must be performed on echoes that have been aligned in time, thus removing effects due to transducer heave as well as small depth variations over consecutive pings. Echo alignment techniques based on thresholding minima, maxima, or half energy, on low-pass filtering techniques and on carrier frequency phase tracking, have been investigated and their relative merits are discussed. [Work supported by ONR N00014-94-1-0121.]

\section{2:57-3:12 Break}

\section{3:12}

5pUW9. A comparison of the finite-difference time-domain and integral equation methods for scattering from shallow water sediment bottoms. Frank D. Hastings, John B. Schneider, Shira L. Broschat (School of Elec. Eng. and Comput. Sci., Washington State Univ., Pullman, WA 99164-2752, shira@eecs.wsu.edu), and Eric I. Thorsos (Univ. of Washington, Seattle, WA 98105)

Scattering from sediment bottoms has a significant effect on acoustic propagation in shallow water. When shear effects are negligible, the water-sediment interface can be modeled using a fluid-fluid model. In this paper, finite-difference time-domain (FDTD) and integral equation (IE) results are compared for a fluid-fluid rough interface. Both methods are exact in that they make no physical approximations, and both can be used either to benchmark approximate analytical methods or to study the propagation problem directly. The comparison presented verifies the numerical accuracy of the two methods. Scattering strengths are computed using a Monte Carlo average over a set of 50 surfaces. Results for single surface realizations are presented as well. Both single-scale Gaussian and multiscale modified power law (MPL) roughness spectra results are presented. While the MPL spectrum is more realistic for shallow water problems, the Gaussian spectrum gives low scattering levels in the backscatter direction, permitting a stringent test of numerical accuracy. Several different bottom types are considered for which the shear speeds are negligible, and attenuation has not been included. For all cases, the FDTD and IE results are obtained using the same surface realizations. In general, the results agree well. [Work supported by ONR.]

\section{$3: 24$}

5pUW10. Shallow-water in-plane bistatic scattering experiment. D. Vance Crowe, Paul C. Hines, and Patrick J. Barry (Defence Res. Establishment Atlantic, Dartmouth, NS, Canada)

Every reverberation experiment done in shallow water is by its nature, bistatic at long ranges. That is to say, in addition to simple monostatic returns, the received signal is composed of energy arriving along bottom bounce paths for which incident and scattered angles are different. Therefore, it is possible to measure in-plane bistatic scattering strength using a monostatic geometry [Hines, Crowe, and Ellis, J. Acoust. Soc. Am. (in review)]. Noting this, a simple array consisting of a pair of free-flooding-
FM (LFM) pulses was transmitted in four frequency bands over the range 900 to $2100 \mathrm{~Hz}$. The reverberation data between the first bottom return and the first surface return provided estimates of monostatic backscatter strength for grazing angles down to $10^{\circ}$. Data arriving after the firstbottom-first-surface interaction provided estimates of in-plane bistatic scattering strength for pairs of incident and scattered grazing angles $\left(\phi_{i}\right.$, $\left.\phi_{s}\right)$ down to $\left(7^{\circ}, 57^{\circ}\right)$. In this paper, the experiment and data are described in light of the monostatic and bistatic arrivals.

\section{$3: 36$}

5pUW11. Sediment density heterogeneity spectra estimated from digital X-radiographs. Dajun Tang (Appl. Phys. Lab., Univ. of Washington, Seattle, WA 98105, djtang@apl.washington.edu) and Robert A. Wheatcroft (Oregon State Univ., Corvallis, OR 97331)

It has been confirmed that sediment volume heterogeneity is one of the major bottom scattering mechanisms, especially in soft-sediment areas. In most cases, scattering by sediment heterogeneity can be modeled by a first-order perturbations technique. The crucial input to such models is the spectrum of sediment spatial heterogeneity. It is difficult to obtain reliable data to estimate such spectra, however, because (1) two-dimensional data sets collected from statistically similar sites are needed, and (2) the data need to cover several orders of magnitude in spatial dimensions. Digital $\mathrm{X}$-radiographs of sediment cores provide an ideal form of data for estimating the spectrum of sub-bottom density variability. Here the data processing procedures will be provided in detail, and physical insights into the resultant spectra discussed. Last, bistatic scattering coefficients resulting from these spectra will be presented.

\section{$3: 48$}

5pUW12. Measurement and modeling of bistatic scattering on the Florida Atlantic coastal shelf. Tokuo Yamamoto, Christopher Day, and Murat Kuru (Univ. of Miami, Div. of Appl. Marine Phys., 4600 Rickenbacker Cswy., Miami, FL 33149)

Measurements of bistatic sea floor scattering on the Fort Pierce, Florida shelf are presented. Scattering measurements have been collected using a new 32 element bilinear acoustic array. According to the volume and roughness scattering models of Yamamoto (1996) and Jackson et al. (1992), bistatic scattering depends on the bottom sediment characteristics, the interface roughness, and the incident and scattered grazing angles. Parameters for the combined volume and roughness scattering model are estimated using the observed bottom roughness and tomographically measured sediment sound speed and attenuation. Observations of bistatic scattering are compared to the combined scattering model. Good agreement between the observed and modeled scattering pattern permits an accurate mapping of mean sound speeds, attenuation, densities, and their threedimensional spatial spectra using observations of bistatic scattering. [Work supported by ONR.]

\section{4:00}

5pUW13. Analysis of laboratory measurements of sound propagating into an unconsolidated water-saturated porous media. Harry $\mathrm{J}$. Simpson and Brian H. Houston (Naval Res. Lab., 4555 Overlook Ave., SW, Washington, DC 20375-5350, simpson@ fugazi.nrl.navy.mil)

A series of measurements in the NRL shallow-water laboratory have been conducted to understand the propagation of sound into and within an unconsolidated water-saturated porous medium. The initial set of measurements was taken to quantify sound penetration as a function of incident angle and interface roughness and to identify the types of waves generated within the water-saturated sandy bottom. These investigations included two-dimensional synthetic array measurements for both a smoothed and a roughened interface. Additionally, above interface and buried source, onedimensional synthetic array measurements were taken to investigate specifically the types of compressional waves generated within the sandy bottom. To complete the physical characterization of the wave speeds 
propagating in the bottom, measurements of the shear wave speeds have been made. These measurements provide a large data base for analysis to understand the physics of this sandy bottom, which consists of a 3.0-mdeep bed of a manufactured 212- $\mu \mathrm{m}$ mean diameter sand. Analysis of these measurements using Biot models and other fluid models is presented. In addition, analysis of enhanced sound penetration at shallow grazing angles due to a roughened interface is discussed. [Work supported by ONR.]

\section{4:12}

5pUW14. The low-frequency radiation and scattering of sound from bubble distributions near the sea surface. William M. Carey (Dept. of Ocean Eng., MIT, Cambridge, MA 02139) and Ronald A. Roy (Boston Univ., Boston, MA 02215)

Microbubble plumes are produced when waves break and are convected to depth by the vortical wave motion. The question is, What role is played by these distributions in the production and scattering of sound near the sea surface at low frequency (LF)? Deep- and shallow-water ambient noise show dramatic increases with wave breaking. Individual wave breaking events show radiation down to $30 \mathrm{~Hz}$. Tipping water-filled trough and bucket experiments show LF radiation associated with the entrainment of known bubble size distributions; suggesting that single bubbles cannot be the cause of this radiation. Microbubble distributions with void fractions greater than $0.01 \%$ act as collective monopole resonant oscillators excited by the energy of formation. Multipole expansions show the radiation is described by a modified Minnaert expression and a dipole radiation pattern (owing to the presence of the sea surface). Since the plume stays put, scattered sound would have a zero Doppler shift with ample Doppler spread. Acoustic images of the sea surface show discrete scattering events, while experiments show LF scattering from free bubble distributions. Results are shown which indicate that bubble plumes from breaking waves not only radiate but scatter LF sound. [Work sponsored by ONR.]

\section{$4: 24$}

5pUW15. Inversion of bistatic reverberation and scattering measurements for seabottom properties. Andrew Rogers, Greg Muncill, and Peter Neumann (Planning Systems, Inc., 7923 Jones Branch Dr., McLean, VA 22102, arogers@plansys.com)

Considerations associated with the inversion of bistatic reverberation and scattering data from a three-dimensional space are discussed. The SCARAB (SCAttering and ReverberAtion from the Bottom) acoustic propagation and scattering model has been the kernel for the study. This model considers three-dimensional, non-plane-wave, range-independent acoustic signals in both mono- and bistatic source receiver configurations. Due to the computationally expensive problem of computing a reverberative field which includes many hybrid acoustic paths and the iterative nature of the inverse process, inversion for seabottom properties requires highly effective search parameter space reduction and algorithm optimization. The parameter space reduction methods and simulated annealing and genetic optimization algorithms utilized are discussed, and their effectiveness measured against several test cases. [Work supported by ONR.]

\section{$4: 36$}

5pUW16. High-frequency sound propagation in shallow water with rough surface scattering. $\mathrm{X}$. Tang, M. Badiey (Marine Studies, Univ. of Delaware, Newark, DE 19716), and J. Simmen (Office of Naval Res., ONR 3210A, Arlington, VA 22217)

Sound propagation at mid to high frequencies $(1-20 \mathrm{kHz})$ in shallowwater environments is extremely complicated, variable, and poorly understood due to multiple processes that cover a wide range of temporal and spatial scales. Numerical simulations using a full-wave PE model are carried out to interpret the acoustic data taken during very carefully designed experiments at a shallow-water site inside the Delaware Bay. Records of broadband acoustic wave time series (from hours to days) are numerically reproduced by using the collected environmental data as input to the $\mathrm{PE}$ model. The statistics of both measured and simulated multipath signals are calculated and compared in the presence of rough sea-surface scattering and surface bubble layer attenuation. The surface wind effects can be separated from the slowly varying volume fluctuations of sound speed, by comparing the temporal variations; while it can be separated from the bottom effects by examining different multipath arrivals. The dependence of coherence on the parameters of wind strength and acoustic frequency is examined.

\section{$4: 48$}

5pUW17. Recent observations of high-frequency acoustic wave propagation in the Delaware Bay. M. Badiey (Marine Studies, Univ. of Delaware, Newark, DE 19716), S. Forsythe (Naval Undersea Warefare Ctr., Newport, RI 02841), J. Simmen (Office of Naval Res., ONR 3210A, Arlington, VA 22217), and X. Tang (Univ. of Delaware, Newark, DE 19716)

Broadband acoustic propagation measurements, as well as complementary environmental measurements including CTD, ADCP, and wind speed, have been conducted in the Delaware Bay during March and September, 1997. With the data from these complete experiments, acoustic pulse travel time and intensity are related to the sound-speed fluctuations in the water column, the sea-surface profile, and the bottom roughness. The direct arrival between acoustic source and receiver contains information about ocean current and temperature, while the arrival corresponding to a single surface bounce contains additional information about the seasurface roughness. Independent measurements of temperature and current allow the sorting of acoustic fluctuations due to ocean volume fluctuations from those due to sea-surface fluctuations. Coherence of the broadband acoustic signals is calculated from the observed data and is found to be correlated with the environmental parameters. 